\title{
ASSESSING THE IMPACT OF SELECT INTESTINAL TRACT CONDITIONS ON EXPRESSION OF LEE ENCODED GENES IN ENTEROHEMORRHAGIC ESCHERICHIA COLI
}

\author{
By: \\ Veronica Cojocari \\ Honors Bachelor of Science in Biology, June 2016 \\ Ryerson University \\ A thesis \\ presented to Ryerson University \\ in partial fulfillment of the \\ requirements for the degree of \\ Master of Science \\ in the program of \\ Molecular Science
}

Toronto, Ontario, Canada, 2019

(C) Veronica Cojocari, 2019 
Author's Declaration for Electronic Submission of a Thesis

I hereby declare that I am the sole author of this thesis. This is a true copy of the thesis, including any required final revisions, as accepted by my examiners.

I authorize Ryerson University to lend this thesis to other institutions or individuals for the purpose of scholarly research.

I further authorize Ryerson University to reproduce this thesis by photocopying or by other means, in total or in part, at the request of other institutions or individuals for the purpose of scholarly research.

I understand that my thesis may be made electronically available to the public. 


\begin{abstract}
ASSESSING THE IMPACT OF SELECT INTESTINAL TRACT CONDITIONS ON EXPRESSION OF LEE ENCODED GENES IN ENTEROHEMORRHAGIC ESCHERICHIA COLI
\end{abstract} Veronica Cojocari, 2019 Master of Science in Molecular Science

Enterohemorrhagic Escherichia coli (EHEC) O157:H7 strain 86-24 accomplishes successful infection upon creating attaching and effacing (A/E) lesions on the enterocytes found in the large intestine. The $\mathrm{A} / \mathrm{E}$ lesion event is possible due to the type III secretion system (T3SS) spanning the EHEC inner and outer membrane. T3SS is a molecular syringe that enables EHEC to establish trans-kingdom interactions with human and other animal hosts. The assembly and regulation of the T3SS is poorly understood. GFP reporter assay experiments showed that short chain fatty acid (SCFA) mixes representative of the large intestine increase the activity of the esp promoter that precedes the espA gene, an important component of the T3SS. Attachment data experiments showed that EspA is essential for EHEC attachment to HEp-2 epithelial cells. The data shows that biochemical cues encountered by EHEC play a role in the regulation and assembly of the T3SS ultimately leading to A/E lesion formation. 


\section{Acknowledgements}

I would like to acknowledge Dr. Debora Barnett Foster for providing me with the opportunity to work on this project. I thank my co-workers from the Foster, Hausner, and McPhee lab: Youn Hee Cho, Sarah Bristonas, Adam Khan, A.J. Francis, Spencer Crook, Jee In Kim and Moussa Diab. Thank you all for helping me with experiments when I needed help, coming to listen to my practice presentations to help me make them better, and being good friends to me. Your friendship and support made graduate school a much better experience than it could have been.

I thank the Molecular Science program, and I especially thank my committee members Dr. Joseph McPhee, Dr. Sarah Sabatinos and Dr. Martina Hausner. 


\section{Dedication}

To my parents Areta and Edward, my brother Dan and my grandmother Anna. For the moral, emotional and, at times technical support which they have always provided and that undoubtedly helped in the creation of this document.

To Goran, for the ceaseless love and support in every endeavor.

To my friends who kept me balanced.

In memory of my grandfather. 


\section{Table of Contents}

Author's Declaration for Electronic Submission of a Thesis .................................................. ii

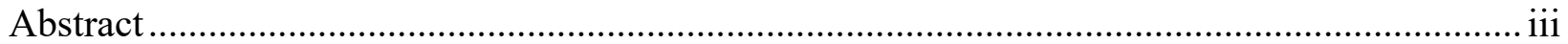

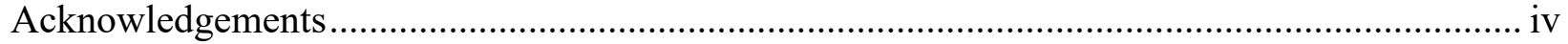

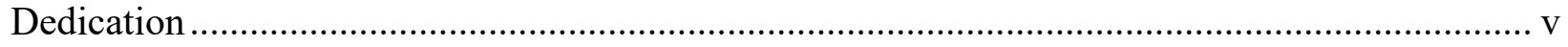

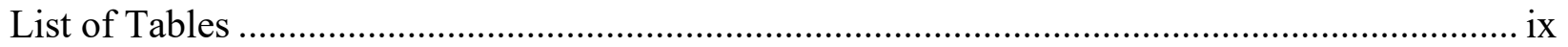

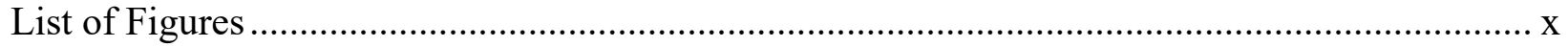

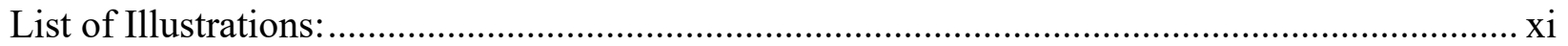

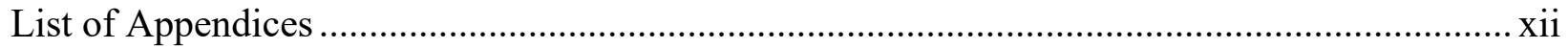

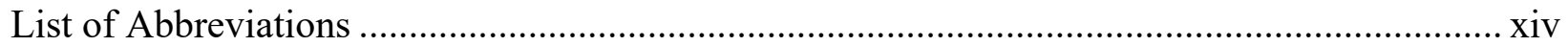

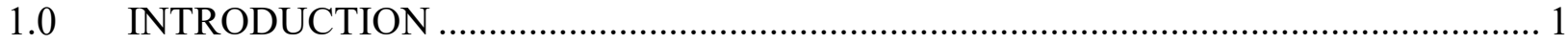

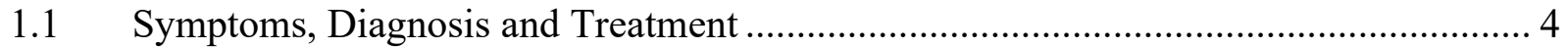

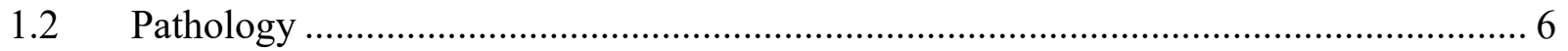

1.3 Enterohemorrhagic Escherichia coli Clinical Isolate 86-24 ..................................... 6

1.4 Virulence Factors of Enterohemorrhagic E. coli ................................................................ 7

1.4.1 Locus of Enterocyte Effacement ........................................................................... 7

1.4.2. Type III Secretion System..................................................................................... 9

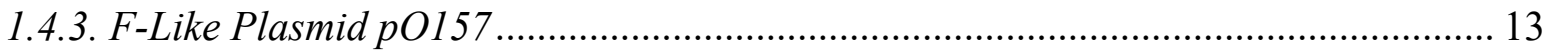

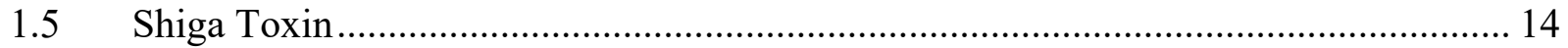

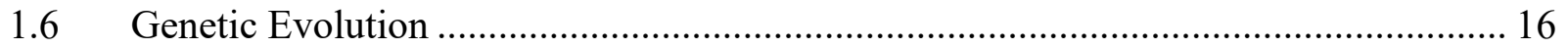

1.7 Host Environmental Cues Influencing EHEC Virulence............................................ 16

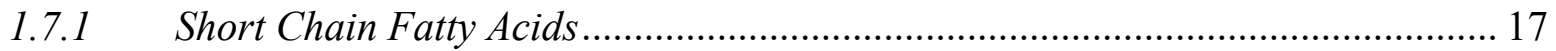

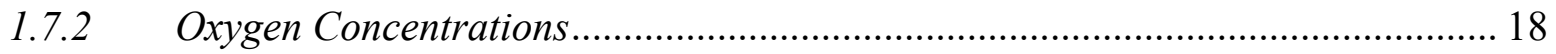

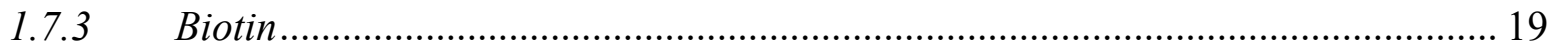

1.8 Regulation Kinetics of Locus of Enterocyte Effacement ........................................... 20

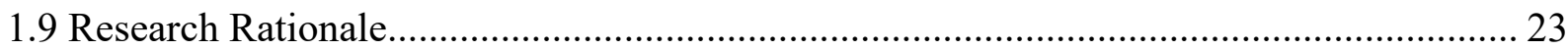

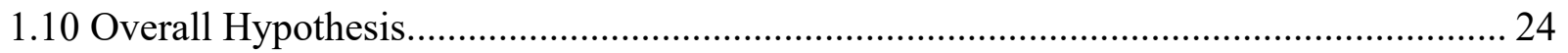

1.11 Specific Hypothesis and Objectives................................................................... 24

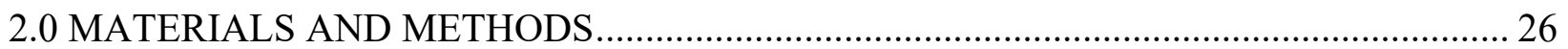

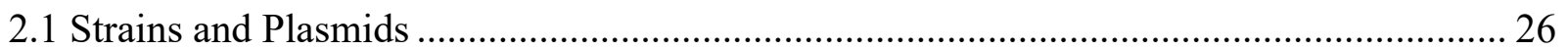

2.2 Isogenic Mutant Construction Using CRISPR-Based Genome Editing .......................... 29

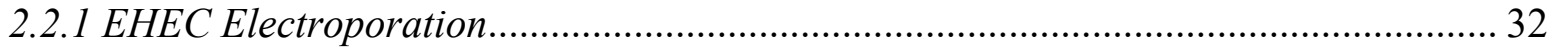

2.2.2 Q5 Site Directed Mutagenesis Kit Protocol............................................................. 32

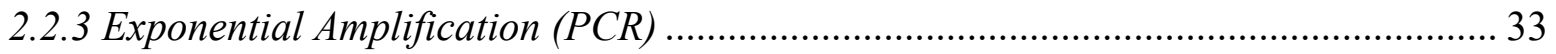




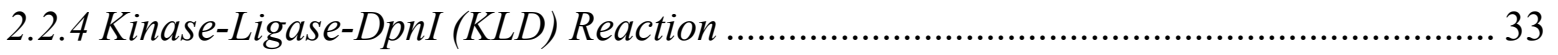

2.2.5 Gene Replacement Protocol ............................................................................. 34

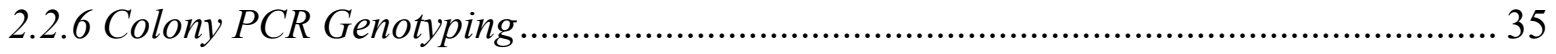

2.2.7 pTargetF Plasmid Curing ..................................................................................... 36

2.2.8 p Cas Plasmid Curing ......................................................................................... 36

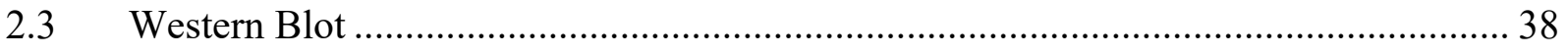

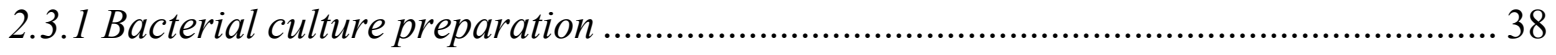

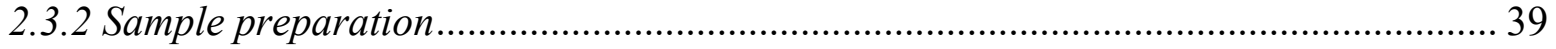

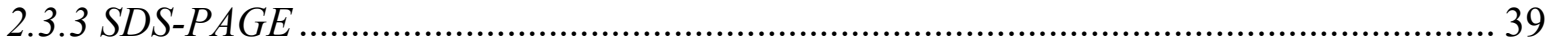

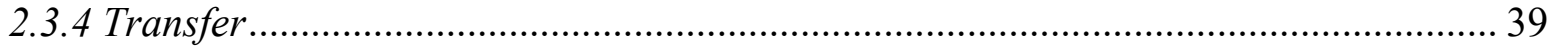

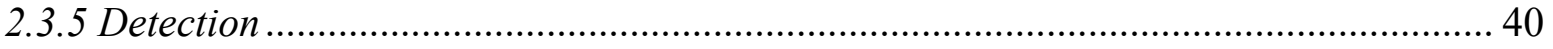

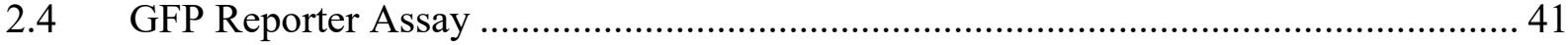

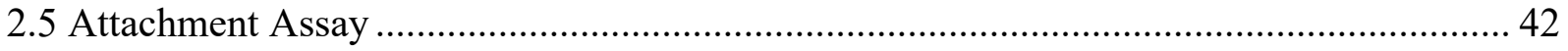

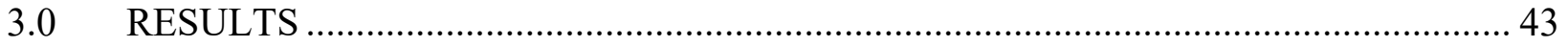

3.1 Isogenic Mutants Showed No Change in Growth Capacity Relative to Wild Type........... 43

3.2 Attachment of EHEC Affected by SCFA and espA Mutation............................................ 45

3.3 Western Blot Analysis of T3SS Proteins ......................................................................... 50

3.4 GFP Reporter Assay of esp Promoter Activity ………...................................................... 52

3.4.1 Activity of esp Promoter Increases at $172 \mathrm{mM}$ SCFA in Low Glucose DMEM High

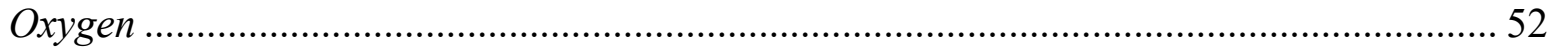

3.4.2 Activity of esp Promoter Increases at $172 \mathrm{mM}$ SCFA in Low Glucose DMEM Low

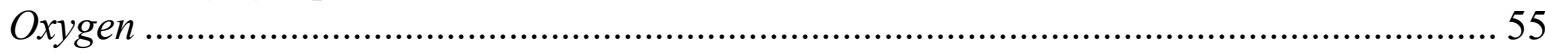

3.4.3 Activity of esp Promoter Increases at 172 mM SCFA in High Glucose DMEM High Oxygen ......................................................................................................... 57

3.4.4 Activity of esp Promoter Increases at 172 mM SCFA in High Glucose DMEM Low

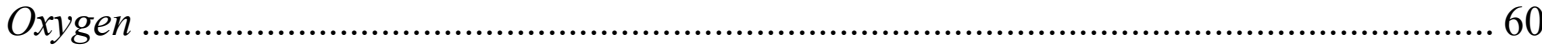

3.4.5 Activity of esp Promoter Increases at 172 mM SCFA in M9 Media with Low

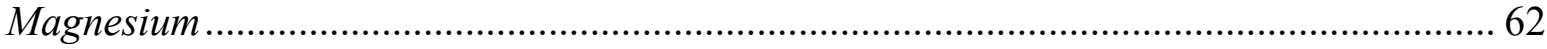

3.4.6 Activity of esp Promoter Increases at 172 mM SCFA in M9 Media with High

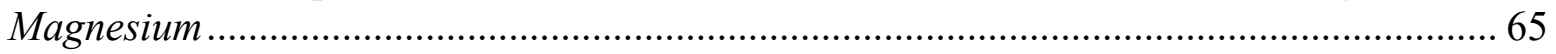

3.4.7 Activity of esp Promoter Increases due to Higher Glucose in $\mathrm{H}_{2} \mathrm{O}_{2}$.......................... 68

3.4.8 Activity of esp Promoter Increases in Low Biotin and Restricted $O_{2}$ Environment..... 71

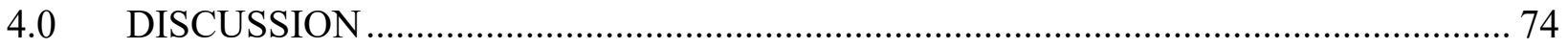

4.1 EHEC Attachment Assays Reveal Significance of EspA Protein for Attachment............. 76

4.2 EspA Protein Levels Remain Constant Across SCFA Treatments..................................... 80

4.3 Modulation of esp Promoter Activity by Selected Culture Conditions .............................. 80 
4.3.1 Impact of Low Glucose DMEM Culture on esp Promoter Activity ........................... 81

4.3.2 Impact of High Glucose DMEM Culture on esp Promoter Activity .......................... 83

4.3.3 Biotin Status Modulates esp Promoter in Low Oxygen .......................................... 86

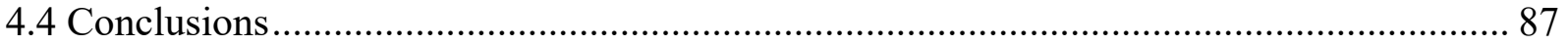

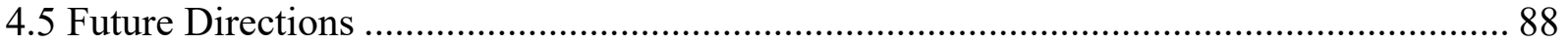

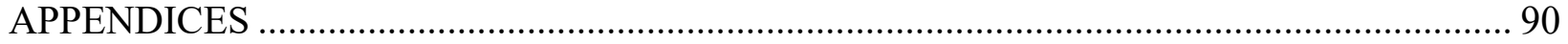

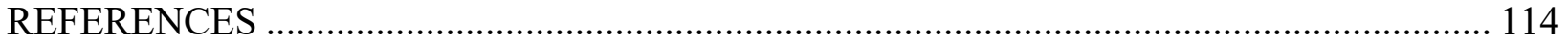




\section{List of Tables}

Table I: E. coli Strains and Plasmids Used in the Molecular Experiments ............................ 28

Table II: Primer Sequences for CRIPSR Gene Editing ................................................... 35

Table III: Primers used in CRISPR/Cas9 for espA Gene Mutagenesis ................................... 37 


\section{List of Figures}

Figure 1. A timeline for EHEC infection pathophysiology ................................................... 4

Figure 2. The locus of enterocyte effacement in EHEC …................................................ 9

Figure 3. Type III Secretion System in EHEC O157:H7 ................................................... 11

Figure 4. Structure of $\mathrm{AB}_{5}$ cytotoxin, Stx2 from EHEC O157:H7 ...................................... 15

Figure 5. Deletion of espA, ler, or espA and ler does not affect EHEC growth.......................44

Figure 6. Attachment of EHEC O157:H7 under various environmental conditions ................. 45

Figure 7. Attachment of various EHEC mutants compared................................................. 47

Figure 8. Expression of EspA protein through western blotting........................................ 51

Figure 9. Activity of the esp promoter is greater under large intestinal SCFA condition .......... 54

Figure 10. The esp promoter is most active under the large intestinal SCFA condition despite

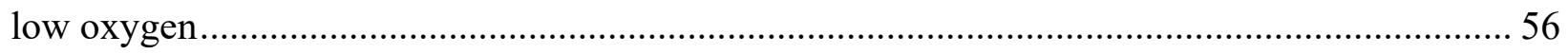

Figure 11. Activity of esp promoter is greatest under the large intestinal condition with high

glucose 59

Figure 12. Activity of esp promoter in high glucose DMEM, low oxygen and various SCFA... 61

Figure 13. esp promoter activity under the influence of low magnesium and SCFA............... 64

Figure 14. esp promoter activity under the influence of high magnesium and SCFA.............. 67

Figure 15. Effect of reactive oxygen species on esp promoter activity ................................. 70

Figure 16. Effect of biotin on esp promoter activity ..................................................... 72

Figure 17. Heat map of GFP reporter data of esp promoter activity in EHEC O157:H7 .......... 73 


\section{List of Illustrations:}

Figure 1. A timeline for EHEC infection pathophysiology ……................................................ 4

Figure 2. The locus of enterocyte effacement in EHEC ............................................................. 9

Figure 3. Type III Secretion System in EHEC O157:H7 …………………………………...... 11

Figure 4. Structure of $\mathrm{AB}_{5}$ cytotoxin, Stx2 from EHEC O157:H7 ………………………….... 15 


\section{List of Appendices}

Appendix A - CRIPSR/Cas9 Mutagenesis of EHEC O157:H7 ............................................ 90

Figure 18. EHEC 86-24 $\Delta \operatorname{esp} A$ mutants............................................................................ 90

Figure 19. Colony PCR of Stop and Delete $\operatorname{esp} A$ Mutants ................................................... 91

Figure 20. Mutant Screening using size shift oligomers................................................. 92

Figure 21. Mutant Screening using size shift oligomers................................................ 93

Figure 22. Potential deletion mutant colonies on selective plates in genes eae and ler ............. 94

Figure 23. Sequencing Data of 86-24_espA Deletion Mutant.............................................. 95

Figure 24.Sequencing Data for the EHEC 86-24_espA stop codon insertion mutant................ 96

Figure 25. RT-qPCR Data Using 16SrRNA as the Housekeeping Gene.................................. 97

Appendix B - Gene Analysis of T3SS Genes Using qRT-PCR ......................................... 97

Figure 26. Raw $\mathrm{Ct}$ values testing the primers for various housekeeping genes in cDNA from

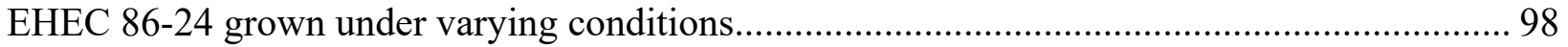

Figure 27. qRT-PCR of LEE gene expression under varying conditions ............................... 99

Appendix C - Western Blot Analysis of T3SS Genes ....................................................... 100

Figure 28. First biological replicate of EspA ( $25 \mathrm{kDa})$ expression under various conditions ... 100

Figure 29. Second Biological replicate of EspA (25 kDa) expression under various conditions.

Figure 30. Third biological replicate of EspA $(25 \mathrm{kDa})$ abundance in cell lysates under various conditions 102

Figure 31. Intimin protein expression as analyzed through Western Blotting........................ 103

Figure 32. EspA Protein expression as observed under static condition with 5\% CO2 .......... 104

Appendix D - Gene Analysis of T3SS Genes Using GFP Reporter Assay ............................ 105

Figure 33. Sequence Data of the various PCR products to form the espA-GFP construct ........ 105

Figure 34. Sequencing data for 3 bacterial strains WT, and $\triangle \operatorname{esp} A$ containing pCR2.1-espA-GFP 106

Figure 35. LG DMEM High Oxygen Control Subtracted .................................................. 107

Figure 36. LG DMEM Low Oxygen Control Subtracted ............................................... 108

Figure 37. HG DMEM High Oxygen Controls Subtracted ................................................ 109

Figure 38. HG DMEM Low Oxygen Controls Subtracted ................................................ 110 


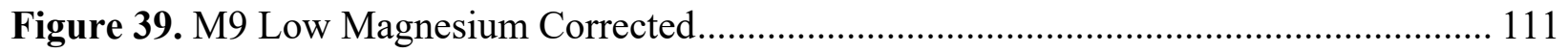

Figure 40. M9 High Magnesium Corrected ................................................................. 112

Figure 41. Reactive Oxygen Species Controls Subtracted ............................................. 113 
List of Abbreviations

$86-24$

$\mathrm{A} / \mathrm{E}$

bp

BSA

CFU

CRISPR

DEAC

DMEM

DNA

dNTP

E. coli

EAEC

EHEC

ELISA

EPEC

EIEC

ETEC

FNR

Gb3

GFP

GI

$\mathrm{HC}$
Enterohemorrhagic E. coli wild-type strain 86-24

Attaching and effacing

Base pair

Bovine serum albumin

Colony forming unit

Clustered Regularly Interspaced Palindromic Repeats

Diffusely adherent $E$. coli

Dulbeco's modified Eagle's medium

Deoxyribonucleic acid

dideoxy-nucleotide triphosphate

Escherichia coli

Enteroaggregative E. coli

Enterohemorrhagic E. coli

Enzyme-linked immunosorbent assay

Enteropathogenic E. coli

Enteroinvasive E. coli

Enterotoxigenic E. coli

Fumirate and Nitrate Reductase

Globotriaosylceramide

Green fluorescence protein

Gastrointestinal

Hemorrhagic colitis 


\begin{tabular}{|c|c|}
\hline HEPES & 4-(2-hydroxyethyl)-1-piperazineethanesulfonic acid \\
\hline HGT & Horizontal Gene Transfer \\
\hline HRP & Horseradish peroxidase \\
\hline HUS & Hemolytic uremic syndrome \\
\hline ID & Infection Dose \\
\hline IS & Insertion Sequence \\
\hline IPTG & Isopropyl $\beta$-D-thiogalactopyranoside \\
\hline $\mathrm{kDa}$ & kilo-dalton \\
\hline LB & Lysogeny Broth \\
\hline LEE & Locus of Enterocyte Effacement \\
\hline Ler & LEE encoded regulator \\
\hline LPS & Lipopolysaccharide \\
\hline LTTR & LysR type transcriptional regulator \\
\hline Map & Mitochondrion associated protein \\
\hline Nle's & Non-LEE encoded effectors \\
\hline O.D. & Optical density \\
\hline $\mathrm{O} / \mathrm{N}$ & Overnight \\
\hline ORF & Open reading frame \\
\hline PAI & Pathogenicity Island \\
\hline PAM & Protospacer adjacent motif \\
\hline PBS & Phosphate-buffered saline \\
\hline PAGE & Polyacrylamide gel electrophoresis \\
\hline PCR & Polymerase chain reaction \\
\hline
\end{tabular}


PE

PRR

PVDF

RNA

SCFA

Stx

T3SS

TBST

Tir

TTP

WT
Phosphatidylethanolamine

Pattern Recognition Receptor

Polyvinylidene fluoride

Ribonucleic acid

Short chain fatty acid

Shiga Toxin

Type 3 Secretion System

Tris-buffered saline tween 20

Translocated intimin receptor

Thrombotic thrombocytopenic purpura

Wild type 


\subsection{INTRODUCTION}

Escherichia coli is a Gram-negative facultative anaerobic bacillus that belongs to a family of gamma-proteobacteria named Enterobacteriaceae. Escherichia coli was first identified by German pediatrician and bacteriologist Theodor Escherich in 1885 with an original name of Bacterium coli. ${ }^{1}$ Enterobacteriaceae function as commensal bacteria in the human gastrointestinal tract while also playing the role of pathogens. Besides Escherichia coli, other members of this family include Salmonella, Shigella, and Yersinia. The site of colonization of Escherichia coli (E. coli) is the lower gastrointestinal (GI) tract. Although typically found as a commensal in the GI tract, E. coli can also be the cause of acute gastroenteritis and other infections throughout the mammalian body, particularly in immunocompromised individuals. These pathogenic traits exist due to horizontal gene transfer (HGT) of virulence genes.

Enterohemorrhagic Escherichia coli (EHEC) was first reported by Ripley et al. in 1983. Ripley described EHEC as a rare non-invasive or toxigenic E. coli which was denoted the serotype $\mathrm{O} 157: \mathrm{H} 7$, which was hypothesized to be transmitted through undercooked meat and resulted in symptoms such as severe hemorrhagic colitis. ${ }^{2}$ Serotype O157:H7 letter "O" signifies the specific $\mathrm{O}$ antigen part of the lipopolysaccharide (LPS) found on the bacterial outer membrane, and the " $\mathrm{H}$ " signified the flagellar antigen.

Microorganisms known to cause disease or infect mucosal surfaces such as the mammalian gastrointestinal tract must encounter many environmental stressors. In order to overcome these stressors and achieve successful infection, pathogens have evolved elaborate biological approaches for both detecting alleged stresses, as well as protecting themselves against said pressures. Pathogens gain disease causing traits through their acquisition of additional genetic content through HGT such as plasmids, pathogenicity islands (PAI) and 
phages. E. coli has adapted into 6 distinct strains that are known to cause disease to humans: enterohemorrhagic E. coli (EHEC), enteropathogenic E. coli (EPEC), enteroinvasive E. coli (EIEC), enterotoxigenic E. coli (ETEC), diffusely adherent $E$. coli (DEAC), adherent invasive $E$. coli, and enteroaggregative E. coli (EAEC). ${ }^{3,4}$ The focus of this research dissertation is EHEC. The mechanisms used by enteric pathogens such as EHEC are essential to their survival and thus, understanding the regulation of these systems is an important research goal.

EHEC strain O157:H7 is a Shiga toxin producing serotype of bacteria, some strains of which fall under the broader classification of shigatoxigenic Escherichia coli (STEC). ${ }^{5}$ EHEC is a human pathogen responsible for major outbreaks such as foodborne and waterborne illness that can be acquired through the consumption of contaminated food or beverages, including raw milk. ${ }^{6}$ The symptoms triggered by EHEC infection are typically resolved within 6-8 days in healthy adults. ${ }^{7}$ Infection by EHEC can lead to colonic escherichiosis with symptoms of hemorrhagic diarrhea, stomach cramps, and in severe cases hemolytic uremic syndrome (HUS). ${ }^{6}$ The manifestation of HUS has been shown to cause death in elderly patients, infected children younger than five years of age, and immunocompromised individuals. For the period of 1998 to 2002, EHEC caused approximately 4,854 infections, 2,000 hospitalizations, and 4 deaths in the U.S.. ${ }^{8}$ The mortality and morbidity caused by EHEC can be attributed to two major elements. EHEC has a very low infectious dose (ID) of 10 to 100 individual microorganisms with approximately $2-7 \%$ of infections leading to the complication of HUS. ${ }^{9}$ Secondly, EHEC possesses the genetic sequence for shiga toxin type 1 (Stx1) and shiga toxin type 2 (Stx2), a toxin similar in structure and mechanism of action to ricin, in that it binds to ribosomes to prevent production of proteins in cells. ${ }^{10}$ This toxin can lead to thrombocytopenia, hemolytic anemia, 
acute renal failure, and ultimately death in immunocompromised individuals, young children and older adults. $^{11-14}$

The first reported account of EHEC infection was in 1982 in an event where 47 people experienced bloody diarrhea from consumption of undercooked hamburger meat acquired from various McDonald's restaurants found throughout Michigan and Oregon. Analysis of stool samples revealed the presence of a previously described $E$. coli $\mathrm{O} 157: \mathrm{H} 7$, a cytotoxigenic strain known to cause hemolytic uremic syndrome (HUS). ${ }^{11}$ The most notorious incident of EHEC outbreak, which landed EHEC the label of the "Hamburger E. coli" was the Jack in the Box outbreak of 1993. During this outbreak, over 73 restaurants were involved in contamination, with over 700 people becoming ill, and four child deaths. Despite these two outbreaks being associated with undercooked hamburgers, EHEC infection can occur from a virtually limitless number of sources such as the latest two outbreaks being associated with romaine lettuce ${ }^{15}$, to unpasteurized milk ${ }^{16}$, drinking water ${ }^{17-20}$, juice ${ }^{21}$, and wild meat. ${ }^{22}$ The Walkerton E. coli outbreak that consisted of a contaminated water supply in Walkerton, Ontario, Canada is another significant outbreak that occurred in the year 2000. The Walkerton outbreak resulted in two thousand individuals becoming sick, six of which suffered from death. ${ }^{23}$

Currently, infection with EHEC in humans has no approved treatments. If an individual is infected with EHEC they would be placed on intravenous liquids until the microorganism is cleared from the intestinal tract by the immune system. ${ }^{24}$ Antibiotics are not used in the treatment of EHEC infection due to their ability to trigger the "SOS" response within the pathogen, leading to an increased production of Shiga toxin, and greater damage to tissues high in globotriaosylceramide (Gb3) receptors, such as the kidneys. ${ }^{24}$ 


\subsection{Symptoms, Diagnosis and Treatment}

The incubation period of EHEC infections can take between 3 days to 8 days, at which point patients will begin to develop severe abdominal cramping followed by non-bloody, watery diarrhea. The diarrhea becomes bloody between day 1 to 5 from when symptoms appear, with up to $50 \%$ of patients experiencing mild fever, nausea and vomiting. Bloody diarrhea can persist from 2 to 4 days where the blood can be minimal streaks, to discharge volumes of tablespoons to cups of blood. The bloody diarrhea is the last hallmark of EHEC infection, once the diarrhea passes, patient recovery can commence. HUS develops in up to $10 \%$ of infected patients typically 2-14 days following initial symptoms (Figure 1).

\section{Pathophysiology of EHEC Infection}
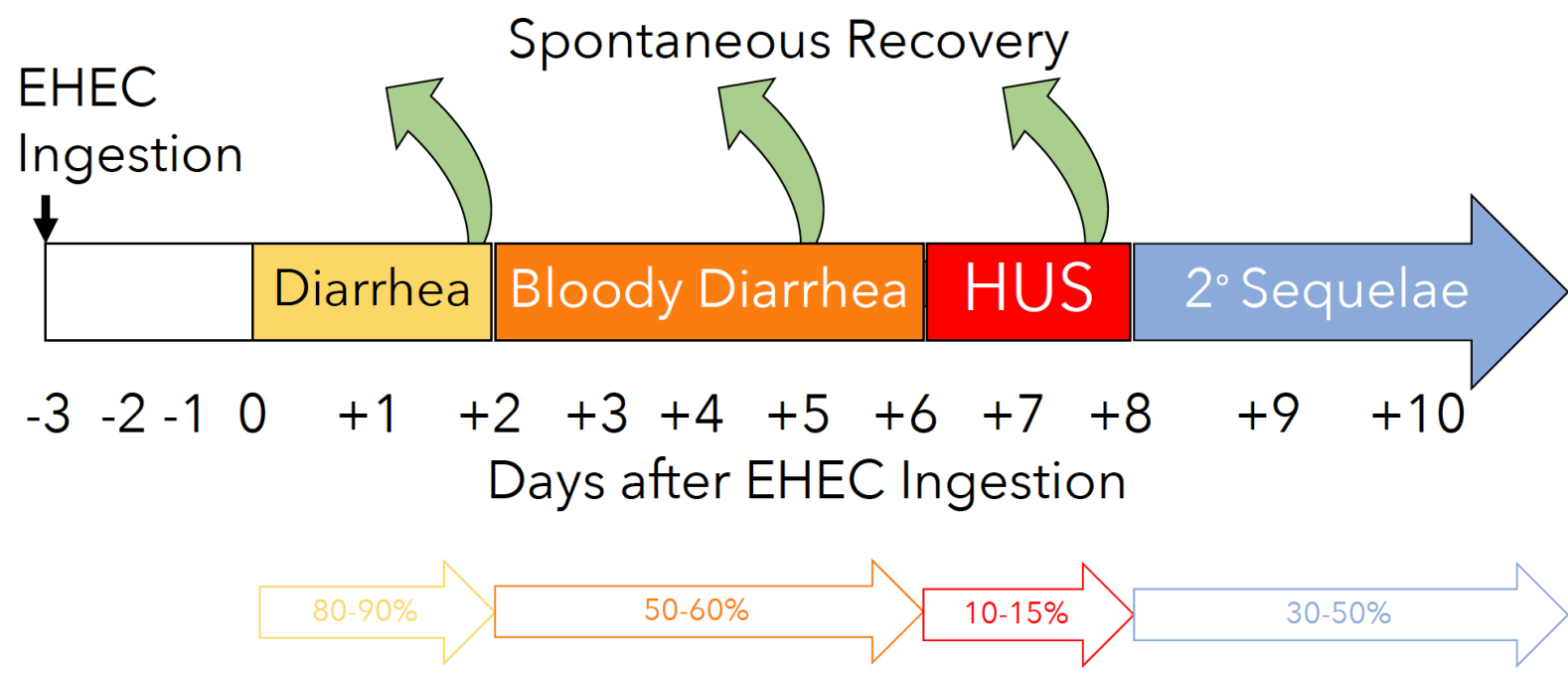

Disease Progression Percentages

\section{Figure 1. A timeline for EHEC infection pathophysiology}

Incubation period of EHEC is generally 3-8 days in length, at which point the development of severe cramps occurs followed by watery diarrhea. Within 1-5 days, the diarrhea becomes bloody and resolves within a week. HUS is developed in approximately $10-15 \%$ of patients 
while about $30-50 \%$ of those individuals go on to experience additional sequelae the likes of neurological complications. Adapted from Scheiring et.al., 2008.

HUS is a complication of STEC infection that primarily occurs in elderly patients. The complication is characterized by thrombocytopenia and thrombotic thrombocytopenic purpura (TTP), miocroangiopathic hemolytic anemia, leading to renal failure and neurological complications that manifest themselves in seizures, coma and hemiparesis. ${ }^{25,26,27}$ The mortality of EHEC infection is approximated to occur in 5-10 \% of patients. The most likely cause of mortality in most EHEC infections is the development of HUS. Permanent neurologic injury and renal failure is observed in up to $5 \%$ of survivors. ${ }^{27-31}$

When a patient becomes infected with EHEC, the clinical diagnosis is done by streaking sorbitol-MacConkey agar with the patient's sample. EHEC's inability to ferment sorbitol will result in white colonies on the sorbitol-MacConkey plates. Other E. coli species are capable of fermenting sorbitol, and when streaked on the sorbitol-MacConkey plates, the colonies would appear red. ${ }^{32}$ Further confirmation of EHEC presence can be done through enzyme-linked immunosorbent assays (ELISAs) that can identify Shiga toxin, $\mathrm{O}$ and $\mathrm{H}$ antigen. A more accurate diagnosis can be further done by polymerase chain reaction (PCR) of EHEC specific genes, followed by sequencing. ${ }^{33-35}$ Although genotyping is unlikely to occur in the hospital setting.

Antibiotic treatment of STEC infection is not recommended as the presence of antibiotics in the GI tract can trigger the expression of Shiga toxin genes which would result in the excretion of Stx into the gut lumen leading to the development of HUS. ${ }^{36-38}$ Antibiotic treatment increases production of Stx due to the presence triggering the SOS response in EHEC. The SOS response is described to induce high-level expression of formerly silent bacteriophage genes, consequently $s t x$ genes. ${ }^{39,40}$ For this reason, treatment involves maintaining support of the patient in three ways: (1) contain the infection to reduce risk of transmission, (2) alleviate the gravity and extent 
of symptoms, and (3) prevent further complications such as HUS. ${ }^{41,42}$ In the severe cases where renal failure develops, the treatment focuses on maintaining fluid and electrolyte balance in the patient, dialysis and anemia treatment. ${ }^{43}$

\subsection{Pathology}

The histopathology of EHEC infected patients comprises of erythema, edema, hemorrhages, and overall mucosal malformations from the rectum to the cecum. ${ }^{43}$ Severe cases of infection result in further intestinal inflammation such as gross fecal peritonitis, toxic megacolon, colonic perforation and necrosis, and rectal prolapse. ${ }^{43}$ The hallmark of EHEC infection is the bacterium's ability to form attaching and effacing (A/E) lesions on colonic epithelial cells. A/E lesions are described by the effacement of brush-border microvilli and cytoskeleton rearrangement of the host cells, causing an actin pedestal to form cupping the bacterium and ultimately creating intimate adherence between bacterial cell and host cell. ${ }^{43}$

\subsection{Enterohemorrhagic Escherichia coli Clinical Isolate 86-24}

Enterohemorrhagic Escherichia coli (EHEC) strain 86-24 is the strain used exclusively in all of the experiments throughout this study. EHEC strain 86-24 is a Stx2 producing strain that was first isolated from an outbreak in Walla Walla, Washington. ${ }^{27}$

EHEC 86-24 produces shiga toxin (Stx) type 2 only, while EDL 933 produces both Stx1 and Stx2. According to epidemiological data Stx2 is shown to be more frequently associated with HUS compared to Stx1. ${ }^{27}$ Experiments suggest that strains expressing only Stx2, such as 86-24, are more neurotrophic in piglets compared to when they become infected with strains expressing both Stx1 and 2, while Stx1 producing strains only induce diarrhea with no signs of kidney damage. $^{27}$ 


\subsection{Virulence Factors of Enterohemorrhagic E. coli}

A successful pathogen requires weapons in its arsenal to combat the immune system's capacity to clear invaders. EHEC contains many such weapons encoded within its 5.5 million base pair circular chromosome, as well as within the large pO157 plasmid, ranges from about 92 to $104 \mathrm{~kb}$. The pO157 carries various mobile genetic elements the likes of transposons, prophages, and insertion sequences (IS). ${ }^{44}$ The limited treatment availability for EHEC infection rationalizes the importance of better understanding the virulence factors used by this pathogen. This understanding can ultimately lead the field into using this arsenal against EHEC by targeting the key proteins with drugs and stopping the infection in its tracks.

EHEC begins coordinate regulation of its virulence gene expression immediately upon the consumption of contaminated food or water. Among the myriad virulence factors produced by EHEC, phage encoded Stx is one of the most detrimental virulence factors to the host, with the type III secretion system (T3SS) a close second. The T3SS is encoded within the locus of enterocyte effacement (LEE) PAI. The means by which the bacteria reaches the host epithelium is through its regulation of flagellation, whereas the LEE encoded T3SS plays a decisive role in the intimate $\mathrm{A} / \mathrm{E}$ lesion formation.

\subsubsection{Locus of Enterocyte Effacement}

A significant genetic factor that contributes to EHEC virulence is the large genomic island named the Locus of Enterocyte Effacement (LEE). The LEE region encodes proteins involved in the formation of attaching-and-effacing (A/E) lesions and consists of five polycistronic operons (LEE1 to LEE5) (Figure 2). ${ }^{45}$ These 5 operons are conserved in all A/E pathogens. The type III secretion system (T3SS) is encoded in LEE1 to LEE3. LEE5 encodes the outer membrane 
adhesin intimin (eae) and the translocated intimin receptor (tir). LEE4 encodes additional T3SS components, both structural and effector in nature. The LEE region varies between EHEC and enteropathogenic Escherichia coli (EPEC) species in the size of the flanking regions, and nonLEE encoded virulence factors. ${ }^{45}$

The core LEE region is $35.4 \mathrm{~kb}$ which is the typical size found in EPEC. ${ }^{46}$ The LEE varies in size due to the flanking regions encoding many more proteins associated with attaching and effacing and can reach up to $110 \mathrm{~kb}$ in other EPEC, ATEC and EHEC isolates. The core LEE region is known to encode the building blocks of the type III secretion system (T3SS), chaperones, effector proteins, and regulators. ${ }^{46}$ T3SSs are commonly found in other Gramnegative pathogenic microorganisms besides E. coli, those organisms include; Salmonella, Yersinia, Shigella, Pseudomonas, Xanthomonas and many others. Within the LEE, genes that encode for the structural proteins making up the T3SS are generally conserved, while effector protein genes are variable. ${ }^{47}$ Horizontal gene transfer has been suggested as a possible mode of acquisition of the entire T3SS gene cluster, explaining its conserved nature. Secreted protein genes such as the effector proteins could be acquired due to distinct events which can explain their variable nature. ${ }^{48}$ LEE flanking regions have a significant number of proteins that amplify a bacteria's virulence such as insertion sequence (IS) elements (eg: IS2, IS3, IS629 and IS630 homologues), prophages (eg: CP4-44, 933L), novel effector genes and so on. ${ }^{47}$ 


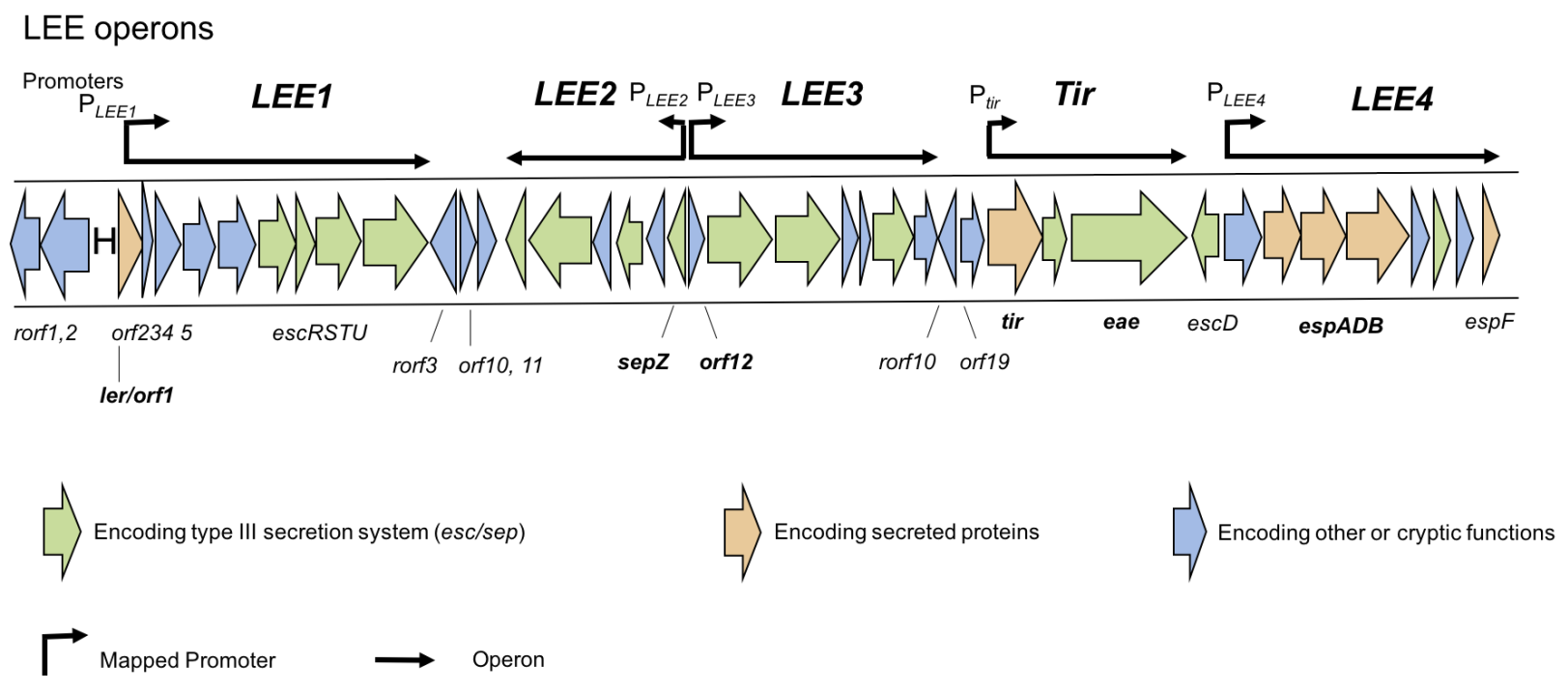

Figure 2. The locus of enterocyte effacement in EHEC

This visual representation of the locus of enterocyte effacement maps out the proteins encoded based on their location in the each of the operons and separates them by the type of protein. There are three kinds of proteins found in the LEE, those that encode for type III secretion system, those encoding secreted proteins and other proteins whose function does not fall under either of the two categories. Adapted from Elliott et.al., 2000.

\subsubsection{Type III Secretion System}

Type three secretion systems are molecular nanomachines used by several pathogenic bacterial strains to translocate effector proteins into host cells in an effort to alter host cellular function with the ultimate goal of facilitating pathogenesis and evading clearance by the immune system. The type III secretion system (T3SS) is an architecturally impressive molecular structure that resembles the shape of a syringe. ${ }^{49}$ This molecular structure is assembled in EHEC when the bacteria has experienced environmental signals that inform the bacterium that it is close to the site of colonization. That being said, it is possible for these large macromolecular complexes to 
be pre-assembled prior to the bacteria reaching the appropriate environment for formation of $\mathrm{A} / \mathrm{E}$ lesions.

The ring structures that span the inner membrane, periplasmic space, through the outer membrane and if successful, the host membrane are comprised of hundreds of unique protein subunits. ${ }^{49}$ The T3SS is a complex bacterial molecular machine utilizing more than a hundred copies of up to twenty different proteins as the building blocks of this multi transmembrane complex (Figure 3). ${ }^{50}$ The resarch focus of this study will be the EspA protein subunits that comprise the needle portion with which EHEC pierces the membrane of human enterocytes, and Ler, the master regulator of the LEE. By knocking out ler and espA, and exposing EHEC to various growth conditions, it will be possible to determine the importance of both the growth conditions and the select proteins involved in the formation of $\mathrm{A} / \mathrm{E}$ lesions. Once the T3SS punctures the intestinal cell membrane, it excretes various effector proteins, one of which is the translocated intimin receptor (Tir) (Figure 3). ${ }^{50-52}$

The coding of $L E E 1$ is responsible for the master regulator ler, the protein which controls LEE2-LEE5 ${ }^{45}$ The basal translocation apparaturs of the T3SS is encoded by LEE2-LEE4, while LEE5 encodes for Tir and intimin. ${ }^{46}$ The T3SS assembly is a multifaceted, yet sequential, process requiring the Sec secretion machinery. The Sec pathway translocates proteins in their unfolded state in a variety of gram-negative and gram-positive bacteria. ${ }^{53}$ There are six components comprising the T3SS: the complex located on the bacterial inner membrane comprised of EscRSTUV, followed by the periplasmic bridge composed of EscJ, the outer 
membrane pore composed of EscC, the outer membrane needle EscF, followed by the EspA filament, and lastly the host pore compled EspBD (Figure 3). ${ }^{54-57}$

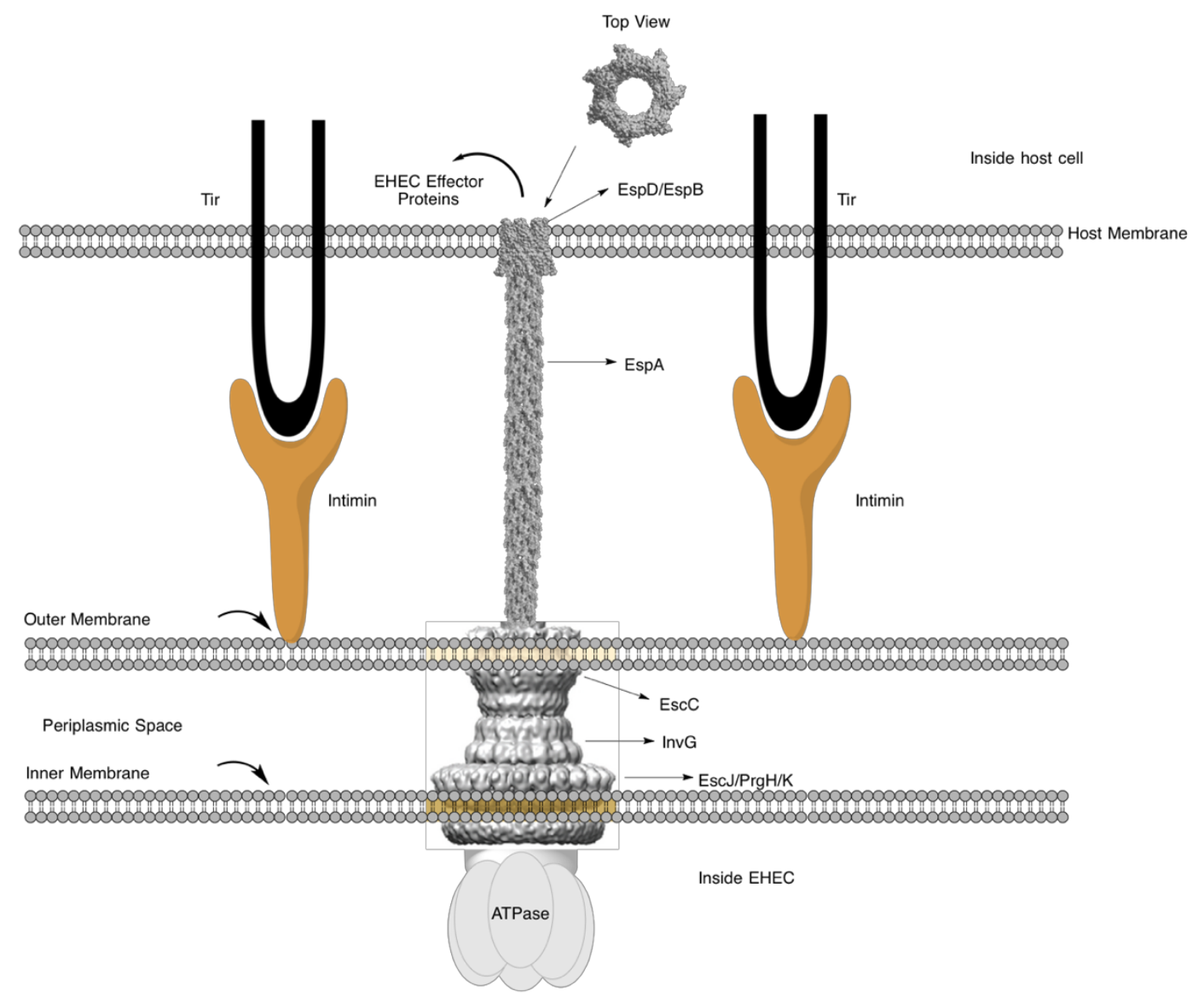

\section{Figure 3. Type III Secretion System in EHEC 0157:H7}

The model above is a depiction of the type III secretion system machinery that is utilized by EHEC O157:H7 in it's pathogenesis. The inner membrane rings comprised of EscJ, PrgH and PrgK subunits span the inner membrane, while the neck comprised of IngG spans the periplasmic space. EscC subunits comprise the ring which spans the outer membrane, anchoring the entire complex in the bacterial cell. The needle subunit was assembled using the Shigella flexneri needle crystal structure with the PDB ID of 2 MME. ${ }^{50,58}$ The intimin in complex with Tir structure was represented as a graphical illustration to better express the interaction. 
The remainder of the proteins encoded by the LEE are not found as structural components of the T3SS, they play a functional role. The three categories of these proteins are as follows: accessory proteins the likes of EscN, SepD and SepL, chaperones such as CesAB, CesD, CesD2, CesF, and CesT, and effectors such as the translocated intimin receptor (Tir). The function of SepD and SepL is unknown, although it has been shown that they interact with one another in order to facilitate protein secretion through the T3SS. ${ }^{59,60,61}$ Protein transport through the T3SS is aided by ATPase EscN. ${ }^{62}$ Chaperones CesF and CesT are involved in the translocation of EspF, Tir, and Map. ${ }^{63-66}$ Chaperone proteins CesAB, CesD and CesD2 are involved in the translocation and assembly of EspA, EspB, and EspD. ${ }^{67-69}$ Conflicting reports with regard to the function of SepL and especially SepD in EPEC and EHEC. ${ }^{70}$ SepD has been reported to be an essential element of T3SS in EPEC, required for both translocator and effector secretion. ${ }^{70}$ Both SepL and SepD are essential for the secretion of translocators but not effectors and that SepL/SepD hierarchy switch from translocator secretion to effector secretion is a conserved feature in all $\mathrm{A} / \mathrm{E}$ pathogens. ${ }^{70}$

The two proteins integral to the formation of A/E lesions, intimin (eae) and Tir (tir) are both encoded in LEE5 (Figure 3). Intimin is a $94 \mathrm{kDa}$ protein that is expressed on the EHEC outer membrane and has a demonstrated role in the colonization and pathogenesis of the strain. ${ }^{71-74}$ The effector Tir, once thought to be a host protein, is translocated through the T3SS into the mammalian host cell. ${ }^{75}$ Once the successful translocation of Tir occurs through the T3SS, the protein embeds itself in the host membrane, with its extracellular domain serving as a docking for the intimin receptor expressed on the bacterial outer membrane. ${ }^{76}$ This allows for the intimate attachment between bacterial cell and host, thus leading to the trademark of EHEC infection, the $\mathrm{A} / \mathrm{E}$ lesions on colonic epithelial cells. ${ }^{76}$ While Tir is produced, secreted and 
utilized in $\mathrm{A} / \mathrm{E}$ lesion formation by both EHEC and EPEC, the two protein isoforms are functionally different. ${ }^{77,78}$ The only protein required for pedestal formation in EPEC infection is Tir. Tir becomes phosphorylated at Tyr474 and recruits Nck to induce actin polymerization. ${ }^{78-80}$ On the other hand, EHEC Tir does not undergo phosphorylation, rather it interacts with host proteins IRTKS and IRSp53 to recruit bacterial effector EspFu, which is required for pedestal formation. ${ }^{81-83} \mathrm{~N}-\mathrm{WASP}$ is recruited by EspFu, this activates Arp2/3 thus stimulating actin nucleation..$^{84,85}$

Other LEE encoded translocated effectors required for both EHEC and EPEC pathogenicity are: Map, EspF, EspG, EspH, and EspZ. Mitochondrion associated protein (Map) functions in three ways: it disrupts the tight junctions of the colonic epithelium ${ }^{86}$, it inhibits the function of the mitochondria ${ }^{87,88}$, and it affects the assembly and arrangement of actin. ${ }^{89} \mathrm{EspF}$ also has three known functions: it disrupts the epithelial tight junctions, it localizes to the mitochondria and is thought to be involved in the induction of apoptosis, and it is hypothesized to affect intestinal ion exchange and thus stimulate diarrhea. ${ }^{90}$ EspZ function is unknown, EspG and EspH however are thought to affect the actin cytoskeleton. ${ }^{91}$

\subsubsection{F-Like Plasmid pO157}

One of the most virulent and prevalent strains of $E$. coli is EHEC O157:H7, where the " $\mathrm{H}$ " refers to the $7^{\text {th }} \mathrm{H}$ antigen while the "O" in the name refers to the $157^{\text {th }} \mathrm{O}$-antigen. ${ }^{53}$ This plasmid contains 100 reputed open reading frames (ORFs), of which 19 have been confirmed as supposed virulence causes. ${ }^{92}$ EHEC pathogenicity was revealed to be modulated by the pO157 plasmid such that the colonization and persistence in bovines is increased ${ }^{93,94}$, epithelial cell adherence is up ${ }^{95}$, modulation of biofilm formation and surface adherence is also elevated ${ }^{96}$, and likelihood of developing hemorrhagic colitis is amplified. ${ }^{97}$ The pO157 plasmid contains the genetic transcript of the Type II Secretion System (T2SS) that has been associated with improved intestinal 
colonization of EHEC in animal models. ${ }^{98}$ Some potential virulence factors found on the plasmid include but are not limited to: etpCDEFGHIJKLMNO, katP, espP, ecf, toxB, stcE, and $e h x A .{ }^{99}$ StcE is the most characterized protein known as a metalloprotease that has been shown to cleave mucintype glycoproteins leading to alteration of cellular response through neutrophil mediated inflammation. ${ }^{100}$ Lastly, an important virulence factor contained within the pO157 plasmid includes enterohemolysins, exotoxins released by EHEC which result in the lysis of red blood cells through the destruction of their membrane. ${ }^{101}$

\subsection{Shiga Toxin}

In STEC infection, the causative agent of cell death and HUS is the phage-encoded Stx. ${ }^{101}$ The extent of Stx production by a given EHEC bacterium is tightly associated with the gravity of malady. ${ }^{102}$ The two major subgroups present in the Stx family are Stx 1 and Stx2, where $57 \%$ of the similarity is shared between the A subunits of the two subgroups, and $60 \%$ of similarity is shared between the B subunits of the two subgroups. ${ }^{103,104}$ EHEC strain 86-24 (used throughout this report) lacks the gene for Stx1, the Stx antigenically indistinguishable to Shigella dysenteriae Stx type $1 .{ }^{105}$ The strain $86-24$ does, however, contain the antigenically unique Stx2 (Figure 4). ${ }^{106}$ Despite the 10-fold greater affinity of Stx1 to eukaryotic cells ${ }^{106}$, Stx2 has been

proven to be 1000 times more cytotoxic in tissue culture compared to Stx $1{ }^{107}$, and Stx 2 is 7 fold more highly associated with HUS in patients infected with EHEC. ${ }^{108}$

The temperate bacteriophage $933 \mathrm{~W}$ in the EHEC genome encodes the stx gene. Expression of the $933 \mathrm{~W}$ prophage is regulated by the cI repressor that typically keeps the stx gene in an inactive state. ${ }^{109-111}$ When the bacteria are exposed to antibiotics or other severe stressors which may cause DNA damage, it enters an SOS response which produces ssDNA, leading to an increase in RecA activity. The cellular DNA repair repressor LexA experiences autocleavage is 
induced by the activated RecA, resulting in activation of cellular DNA repair machinery expression. ${ }^{12,113}$ Simultaneously, RecA cleaves the $\lambda$ prophage repressor cI leading to the stimulation of the phage lytic cycle ${ }^{114}$ and translation of the stx gene into Stx protein. ${ }^{114}$ The bacterial SOS response is typically induced by antibiotics, for this reason, antibiotics are discouraged in treatment of EHEC infection as their use may result in the increase of Stx expression thus leading to the development of HUS. ${ }^{115}$

A)

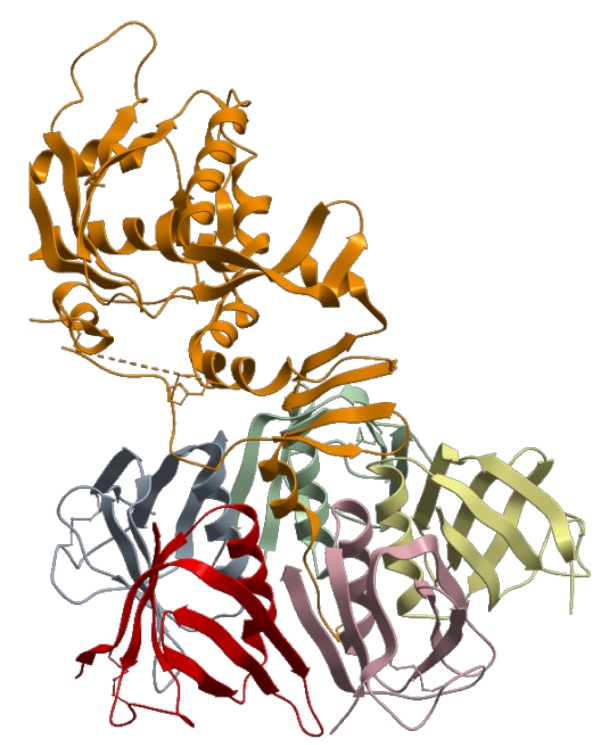

B)

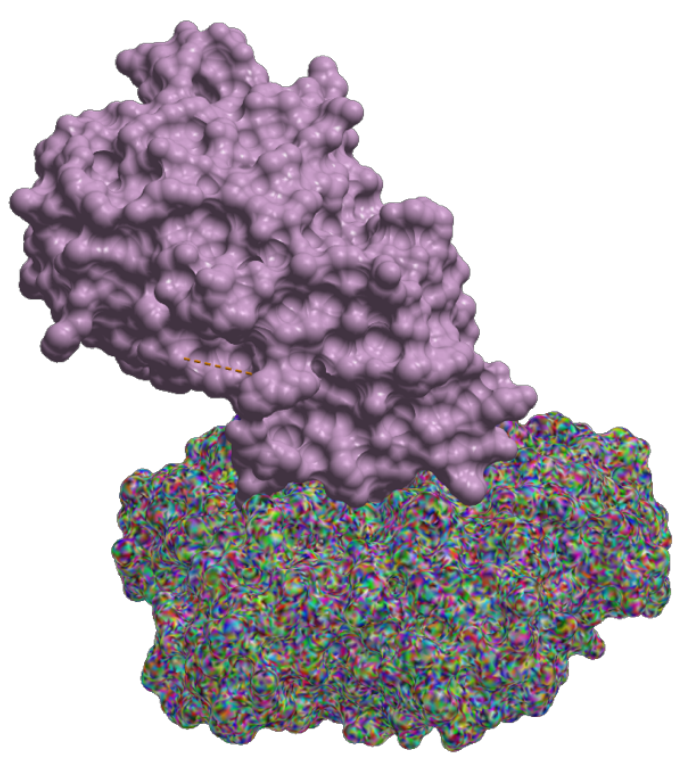

\section{Figure 4. Structure of $\mathrm{AB}_{5}$ cytotoxin, Stx2 from EHEC 0157:H7}

The images above are a ribbon representation (A) and a mesh representation (B) of the crystallized StxAB5 toxin released by EHEC O157:H7. A0 Coloured in orange is the catalytically active A subunit with the B pentamers attached in red, pink, yellow, green and blue. B) The mesh representation of this endotoxin is labelled with two different colours. The top portion in purple represent the A subunit that shares $57 \%$ similarity with the Stx 1 A subunit. The bottom portion in multicolour is the B subunit that shares $60 \%$ similarity with the Stx1 B subunit. Adapted from Fraser et.al., 1994. 


\subsection{Genetic Evolution}

The origin of EHEC is believed to stem from a divergence from a common laboratory strain, E. coli $\mathrm{K}-12 .{ }^{116}$ The $\mathrm{K}-12$ genome is 4.1 megabases, to which evolution into EHEC has added approximately 1.4 megabases leading to an approximate EHEC genome size of 5.5 megabases. The aforementioned 1.4 megabases of genetic material has been acquired through horizonal gene transfer and can be found scattered throughout the EHEC genome in what are referred to as Oislands that contain PAIs and virulence factors. ${ }^{117,118}$ EHEC possesses the 4.1 megabase backbone of K-12, however, the most recent common ancestor of EHEC O157:H7 is believed to be EPEC O55-H7. ${ }^{119}$

\subsection{Host Environmental Cues Influencing EHEC Virulence}

Bacterial pathogens such as EHEC are subject to profound environmental fluctuations due to their transmission from the outside environment on contaminated food or beverage, to the host they will ultimately infect. Through these transitions, EHEC experiences variation in gene expression patterns in anticipation of achieving niche specificity and guaranteeing energy efficiency essential to survival. EHEC is expert at surviving in a variety of environmental niches, capable of colonizing the GI tract of both ruminant and human hosts. The virulence genes involved in successful colonization of human hosts involve the use of the type III secretion system (T3SS), type III secreted effector proteins, including Tir (translocated intimin receptor) and intimin. ${ }^{120,121}$ All these virulence factors, and many others, are encoded within the pathogenicity island (PI) named the Locus of Enterocyte Effacement (LEE). LEE expression is a complex and synchronized process sensitive to many environmental signals. Some important environmental cues that modulate the expression of EHEC virulence factors to consider involve 
but not are partial to: temperature, $\mathrm{pH}$, bicarbonate, osmolarity, growth phase, bile salts, ethanolamine (EA), microbial flora metabolites, epinephrine and norepinephrine, and oxygen availability. ${ }^{122,123}$

Acid stress is encountered by EHEC in the stomach, where the bacteria must be able to withstand this stress for approximately 2.5 hours in order to survive and continue to the site of

colonization. ${ }^{124,122,125}$ Bile salts are encountered in the duodenal portion of the small intestine. ${ }^{123}$ Research suggests that bile salts act as an environmental cue for EHEC to elicit protective modifications to its outer membrane to ensure successful passage through the small intestine to the site of infection. ${ }^{126,127}$ Ethanolamine is produced through the breakdown of phosphatidylethanolamine (PE) during the process of intestinal epithelial turnover. ${ }^{128}$ EHEC binds to PE, where PE acts as receptor for EHEC thus increasing bacterial adhesion to host cells. ${ }^{128}$ Commensal bacterial metabolites such as short chain fatty acids (SCFA) have been shown to be a powerful cue for EHEC, where low concentrations of SCFA associated with the small intestine were shown to increase motility and motility associated genes. ${ }^{129}$ Host hormones such as epinephrine and norepinephrine have been shown to be utilized by EHEC and other pathogens as signals for differential modulation of a variety of virulence factors. ${ }^{125,130}$

\subsubsection{Short Chain Fatty Acids}

As EHEC traverses the gastrointestinal tract, it is bound to encounter many different microbial communities. ${ }^{131}$ These commensal communities differ in size as mentioned in the section regarding the various portions of the intestinal tract. The small intestine contains a smaller microbial community, and a distinct microbial profile than the large intestine. ${ }^{132-134}$ Some metabolites found in the GI tract are composed of three principal SCFA; acetate, propionate and butyrate, and their concentrations and composition fluctuate from the small 
intestine to the large intestine. Lower concentrations of SCFAs are typically found in the ileum while larger concentrations $(>50 \mathrm{mM})$ are typically found in the colon. ${ }^{132-134}$ This research explored the effect of SCFA concentrations and compositions associated with the small intestine and effect of SCFA concentrations and compositions associated with the large intestine to examine if there are differences in the expression of LEE encoded T3SS genes. Particularly, the proteins of interest are Ler the master regulator of LEE, and EspA the building block of the needle portion of the T3SS molecular syringe.

\subsubsection{Oxygen Concentrations}

The gastrointestinal tract lumen is an increasingly anaerobic environment that may act as a trigger to EHEC as this bacterium traverses the GI tract toward the site of infection. ${ }^{135,136}$ EHEC possesses the ability to sense oxygen concentrations in its environment, this ability allows EHEC to switch between any one of three types of respiration; aerobiosis, anerobiosis or microaerobiosis. The switch is administered by two master regulators; fumirate and nitrate reductase (FNR) for anaerobiosis and ArcA for microaerobiosis. ${ }^{137} \mathrm{ArcA}$ is a response regulator that becomes activated under low oxygen conditions to activate $c y d A B$ (terminal electron acceptor cytochrome $o$ oxidase complex) gene expression, while FNR becomes activated when oxygen levels drop further and is a repressor of $c y d A B .{ }^{138}$ The oxygen sensitive transcription regulator FNR holds control over 100 genes in E. coli. This protein is capable of sensing oxygen availability through the assembly-disassembly of [4Fe-4S] clusters. ${ }^{137} \mathrm{FNR}$ is inactive under aerobic conditions as a monomeric apoprotein and active under anaerobic conditions as a dimeric holoprotein harboring one iron-sulphur cluster per subunit. ${ }^{137}$ In concert, FNR and ArcA allow for the maximum expression of essential terminal acceptor complex in microaerobic 
environments with $2 \%$ oxygen present. ${ }^{138}$ There is little data on the role of oxygen availability and the effects it has on EHEC virulence factors.

\subsubsection{Biotin}

EHEC gene expression is under the control of an array of specific and global regulators all the while being influenced by environmental stimuli. ${ }^{139}$ Two vastly distinct environments experienced by EHEC as it traverses the GI tract are the small intestine and the large intestine. Researchers have identified some of the factors present in the two dissimilar environments and how they might affect EHEC. ${ }^{139-143}$ For instance, low-pH affects EHEC in that it improves the bacterium adhesion to epithelial cells. ${ }^{144}$ Bile-salt stress and low-pH enhances adherence and/or secretion of LEE-encoded effectors by EPEC, while ammonium represses said bacterial responses. ${ }^{140,141}$ Other research has shown that bile-salt treatment has no effect on EHEC LEE gene expression. ${ }^{145}$ For this reason, further investigation is essential into what factors are linked to site specific colonization.

Biotin is an indispensable molecule for all organisms as it performs the role of an essential cofactor for carboxylases and decarboxylases to function appropriately. ${ }^{146}$ Humans cannot synthesize biotin, thus they are only capable of obtaining biotin exclusively through exogenous sources. ${ }^{146}$ The human intestine can encounter two sources of biotin: one of the two sources is dietary in origin, while the other is synthesized by the commensal microorganisms within the GI tract, particularly in the colon. A large proportion of the dietary biotin absorption occurs in the small intestine. ${ }^{146,147}$ For this reason, it is expected that biotin levels are higher in

the small intestine compared to the large intestine. ${ }^{146,147} \mathrm{E}$. coli is capable of tightly regulating biotin synthesis on the basis of supply and demand through the biotin protein ligase, BirA. The enzyme BirA has a two-fold responsibility: one is to funnel biotin into metabolism, and the other 
is to act as a negative transcriptional regulator of the bio operon (biotin synthetic operon) that contains genes bioA and bioBFCD. ${ }^{146,148}$ When E. coli senses biotin levels to have surpassed the physiological requirement, the biotin operon (bio) transcription is repressed via BirA in complex with biotin binding to the biotin operator $($ bioO $) .{ }^{149}$ Studies have revealed that EHEC O157:H7 possesses a regulatory pathway mediated by BirA in concert with biotin that control LEE gene expression and bacterial adherence through Fur signaling. ${ }^{150}$ More specifically, once BirA binds biotin, the BirA-biotin complex bind to the fur promoter, initializing transcription of Fur. EHEC O157:H7 adherence is negatively correlated with biotin concentrations in both the small and large intestines of mice. ${ }^{150}$ Furthermore, mice fed a high-biotin diet experienced abridged adherence of $\mathrm{O} 157$ to the GI epithelium. The biotin regulatory pathway in EHEC is important for site-specific colonization. As a result, biotin may be useful in the prevention of EHEC infection.

\subsection{Regulation Kinetics of Locus of Enterocyte Effacement}

Regulation of the locus of enterocyte effacement (LEE) pathogenicity island in EHEC is a complicated and poorly understood system. The assembly of the T3SS, the molecular nanomachine used by EHEC to infect its host, is thought to be triggered by direct bacterial contact with the host cell. This topic is still not agreed upon in the scientific community and still happens to be a controversial debate. This would imply that the bacteria would have to make its way through the loose outer mucus and the attached inner mucus (seldom penetrated by bacteria) to the enterocytes before the assembly of this molecular machine begins. The environmental factors modulating the T3SS are countless, and many are not defined.

\subsubsection{Post-Transcriptional Regulation of LEE4}

The LEE PAI organization consists of five major operons, LEE1 to LEE5, within which all the essential virulence factors are encoded, from the T3SS, chaperones, secreted proteins and 
regulators. LEE4 is the operon that encodes the proteins responsible for the translocation of the secreted effectors into the host cell: EspA, EspB, EspD, CesD2 (a chaperone for EspD), effector EspF, L0017 or Orf29 (EspA chaperone) and a T3SS component (EscF). ${ }^{87,151-153}$ The function of EspA is to polymerize with itself to form a long cylindrical filament (Figure 3) extending from the base of the T3SS located at the bacterial surface to the host cell membrane up to $600 \mathrm{~nm}$ in length. ${ }^{154-156}$ According to research done in enteropathogenic Escherichia coli (EPEC) the base of the EspA filament that extends from the outer membrane ring is presumably composed of EscF. ${ }^{157,158} \mathrm{EspD}$ if found on the latter end of the EspA filament, where it interacts with the host cell membrane to from a pore, this pore acts as a trans-kingdom portal that allows the translocation of effector proteins with the help of EspB. ${ }^{159,160} \mathrm{EspB}$ aids in pore formation and interacts with myosins within the host cells with the goal of forming microvillus lesions and preventing phagocytic degradation of EHEC. ${ }^{161-163}$

The first protein encoded within the LEE4 operon is SepL, a non-secreted protein immediately upstream of the $\operatorname{esp} A$ gene, with a conserved sequence identity of $90 \%$ among $\mathrm{A} / \mathrm{E}$ pathogens. A sepL isogenic mutant behaves in a way that prevent the formation of EspA filaments, A/E lesions and contain nearly undetectable levels of secreted translocator proteins yet strangely possesses an amplified secretion of effector proteins. ${ }^{70,164,165} \mathrm{SepL}$ is involved in a molecular switch mechanism with SepD (another T3SS component) where the two are thought to control the secretion of T3SS effectors and translocators. ${ }^{164}$ Research revealed that SepL plays an important role in timing the secretion of the translocated intimin receptor (Tir) by binding to Tir and preventing the secretion of this effector until all the necessary components for translocation have been exported. ${ }^{166}$ The mechanism by which SepL binds to SepD, Tir or any other proteins and regulates secretion kinetics is poorly understood. 
Gene transcription of $\operatorname{esp} A D B$ in EHEC was thought to be associated with the promoter esp found at the end of the $s e p L$ coding region, just before the espA gene origin. ${ }^{165,167,168} \mathrm{SepL}$ protein was thought to be transcribed separately from the esp $A D B$ gene region. This was rationale behind the experiments in this study, as the research was conducted based upon this assumption. The esp promoter was assessed in this study, assuming the promoter regulates activity of espA in EHEC 0157:H7 strain 86-24. Newer research has elucidated the fact that the gene sepL in EPEC is co-transcribed laterally with the remainder of the LEE4 genes. ${ }^{45}$ Furthermore, experiments assessing chromosomal lacZ transcription to test esp promoter activity in various EHEC strains failed to detect activity in the esp promoter. ${ }^{169}$ Northern blot analysis detected a $3 \mathrm{~kb}$ transcript for $\operatorname{esp} A$ and $\operatorname{esp} D$, elucidating that the two genes may be cotranscribed. Collectively, these studies revealed the possibility of an upstream promoter located at the beginning of the sepL coding region that may be responsible for the transcription of the LEE4 operon. In which case espADB mRNA could be generated through post-transcriptional processing in EHEC. Additional studies showed that espADB mRNA is produced through posttranscriptional processing of the LEE4 operon in EHEC. This proved that $\operatorname{sepL}$ is a gene that belongs to the LEE4 operon in EHEC, a previously unknown fact. ${ }^{170}$ Moreover, LEE4 processing occurs in an E. coli $\mathrm{K}-12$ background due to $\mathrm{K}-12$ possessing RNaseE. RNase $\mathrm{E}$ is the molecule central to RNA metabolism. This enzyme plays important roles in degradation, processing and maturation of various RNA molecules and is responsible for the cleavage of LEE4 into the subsequent gene products. ${ }^{170}$ These findings have critical ramifications on the realm of T3SS regulation, sepL and $\operatorname{esp} A D B$ gene expression. 


\subsection{Research Rationale}

Previous research has proven that short chain fatty acid (SCFA) concentrations modulate the expression of flagellar genes implicated in motility. ${ }^{171}$ The trend being that under SCFA mixes typical of the small intestine the flagellar expression is up-regulated when compared to treatment with SCFA mixture typical of the large intestine. ${ }^{130}$ The results suggested that external biochemical conditions are sensed by EHEC and can trigger expression of specific genes, such as motility genes to enhance bacterial motility through the small intestine to the large intestine, the site of infection. Since LEE-encoded virulence factors participate in the formation of A/E lesions during colonization, it is possible that expression of these factors is reciprocally regulated relative to flagella in response to these host intestinal factors. It is expected that the genes of interest espA and ler, both of which participate in establishing successful colonization, should be up-regulated by exposure to conditions that mimic those of the human large intestine. By

contrast, it is expected that expression of these genes should be either unchanged or down regulated by exposure to cognate conditions that mimic the small intestine. 


\subsection{Overall Hypothesis}

EHEC exposure to large intestinal micro environmental conditions up-regulates the expression of LEE-encoded virulence factors that promote the formation of $\mathrm{A} / \mathrm{E}$ lesions at the site of infection.

\subsection{Specific Hypothesis and Objectives}

\subsubsection{Hypothesis I}

Expression of LEE-encoded virulence genes eae, espA, and ler will be increased in response to conditions that mimic in the lumen of the large intestine relative to the small intestine.

\subsubsection{Objective I}

Generate transcriptional/translational GFP reporter fusions for the genes eae, espA and ler in EHEC 86-24

CRISPR-Cas9 was used in wild type EHEC to insert eGFP with flanking regions homologous to the gene of interest right after the first codon of the genes ler, eae, and espA. These GFP engineered EHEC will be exposed to a vast array of varying conditions such as 30 mM SCFA mix, $172 \mathrm{mM}$ SCFA mix, $\mathrm{NO}_{2}, \mathrm{NO}_{3}$, low oxygen etc. The fluorescence as well as the growth of the bacteria will be monitored over a select period of time. This will reveal great insight into the effect of varying conditions on the genes of interest. 


\subsubsection{Objective II}

Assess expression of the GFP reporter in each of the reporter fusions in EHEC 86-24 after treatment with each of the following treatments: $30 \mathrm{mM}$ SCFA, $172 \mathrm{mM} \mathrm{SCFA,} 30 \mathrm{mM}$ $\mathrm{NaCl}, 172 \mathrm{mM} \mathrm{NaCl}, 30 \mathrm{mM}$ SCFA low oxygen, 172mM SCFA low oxygen, $30 \mathrm{mM} \mathrm{NaCl}$ low oxygen, $172 \mathrm{mM} \mathrm{NaCl}$ low oxygen and other relevant conditions that are representative of small molecules found in both the large and small intestine.

\subsubsection{Hypothesis II}

EHEC exposure to conditions that mimic the lumen of the large intestine relative to those of the small intestine will increase the extent of $\mathrm{A} / \mathrm{E}$ lesion formation.

\subsubsection{Objective I}

Assess and compare attachment of EHEC 86-24 under sets of conditions specified in Objective

\subsubsection{Objective II}

The objective of this section is to confirm the changes in expression of the 3 genes observed section 1.8.1.1.1 and confirmed in section 1.8.1.1.2. This will be an evaluation of whether the changes observed at the transcriptional level, and translational level match the functional changes in $\mathrm{A} / \mathrm{E}$ lesion formation particularly for the different SCFA treatments. The CRISPR/Cas9 mutants generated will be used as negative controls in this functional assay. 


\subsection{MATERIALS AND METHODS}

2.1 Strains and Plasmids

All bacterial strains, plasmids, and oligonucleotides used in this study are listed in Table I. All E. coli strains were grown aerobically in Luria-Bertani (LB) broth, M9, DMEM low glucose or DMEM high glucose at $37^{\circ} \mathrm{C}$ unless stated otherwise. When appropriate the media was supplemented with kanamycin $(50 \mu \mathrm{g} / \mathrm{mL})$, spectinomycin $(100 \mu \mathrm{g} / \mathrm{ml})$, and ampicillin (100 $\mu \mathrm{g} / \mathrm{ml})$. Enterohemorrhagic E. coli O157:H7 wild-type strain, 86-24 did not possess any resistance and is referred to as "wild-type" in this study. E. coli DH5 $\alpha$ (New England Biolabs) was used as an intermediate host for all plasmid engineering. The genes of interest were as follows: espA, the gene located on LEE4 operon coding for EspA protein, the structural subunit comprising the translocon of the T3SS, and ler the master regulator of LEE2-LEE5 encoded on $L E E$. The method used to generate the isogenic mutants involved the novel $\underline{\text { Clustered }}$ Regularly Interspaced $\underline{\text { Palindromic }}$ Repeats (CRISPR)-Cas9 method, that was modified for use in EHEC and can be viewed in detail in section 2.3 of the methods section. The pCas containing EHEC 86-24 cells were subjected to induction with $50 \mathrm{mM}$ arabinose to 20 minutes. Once the $\lambda$ red recombination system from the pCas plasmid was induced for 20 minutes at $\mathrm{OD}_{600}$ of 0.6 , the cells were harvested, washed with cold sterile water and $1 \mathrm{mM}$ MOPS, and electroporated with the pTarget $\Delta e s p A$ (or pTarget $\Delta l e r, \mathrm{pTarget} \Delta \operatorname{esp} A \Delta l e r$ ) plasmid and the delete or stop oligo (Table II; Table III) separately. Two cell populations were used to ensure successful mutagenesis in EHEC, one population that received pCas plasmid containing all the recombination machinery, pTarget $\Delta$ gene acting as the CRISPR locus in the cell, and single stranded DNA (ssDNA) that is to be integrated into the chromosome during the recombination process while the 
other population did not receive any ssDNA. The induction of $\mathrm{pCas}$ using arabinose gave rise to necessary protein machinery responsible to $\lambda$ red recombination, as well as translated Cas 9 into its functional enzyme form. The insertion of pTarget $\triangle e s p A$ (via electroporation) into the EHEC 86-24 with activated pCas allowed the Cas9 enzyme acted as a prosthetic CRISPR locus directing Cas9 to the desired target protospacer adjacent motif (PAM) sequence. The ssDNA allowed for successful repair of the double stranded break in the desired gene region. The way in which EHEC mutants were differentiated from unaltered EHEC cells was through the principle that un-repaired double stranded DNA breaks in the bacteria cause cell death. ${ }^{172}$ The cells lacking the ssDNA lacked the necessary component for DNA repair, leading to their death. Therefore, when plating the cells without ssDNA on the double antibiotic plates containing kanamycin $(50 \mu \mathrm{g} / \mathrm{ml})$ to select for pCas and spectinomycin $(100 \mu \mathrm{g} / \mathrm{ml})$ to select for $\mathrm{pT}$ arget $\Delta$ gene lead to no bacterial growth. This was the control to ensure that the presence of $\mathrm{pTarget} \Delta$ gene was accomplishing bacterial killing. The same bacteria that were treated in the same manner but that received ssDNA to be integrated into the chromosomal repair grew colonies. Those colonies were supposed to contain mutants with deletions in the desired gene regions. 
Table I: $E$. coli Strains and Plasmids Used in the Molecular Experiments

\begin{tabular}{|c|c|c|}
\hline \multicolumn{3}{|l|}{ Bacterial Strains } \\
\hline Strain & Description & Reference \\
\hline EHEC 86-24 & $\begin{array}{l}\text { Clinical isolate of O157:H7 serotype of } \\
\text { enterohemorrhagic Escherichia coli }\end{array}$ & \\
\hline DH5 $\alpha$ pTargetF & Carrier of pTargetF plasmid & Addgene \\
\hline DH5 $\alpha$ pCas & Carrier of the pCas plasmid & Addgene \\
\hline EHEC 86-24_esp $A$ & CRISPR/Cas9 Knockout of espA gene & This study \\
\hline EHEC 86-24_esp $A$ pCas & $\begin{array}{l}\text { CRISPR/Cas9 Knockout of esp } A \text { gene } \\
\text { containing pCas for further mutagenesis }\end{array}$ & This study \\
\hline 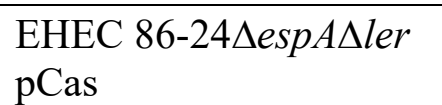 & $\begin{array}{l}\text { CRISPR/Cas9 Knockout of both espA and ler } \\
\text { genes containing pCas for further mutagenesis }\end{array}$ & This study \\
\hline 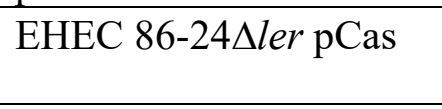 & $\begin{array}{l}\text { CRISPR/Cas9 Knockout of ler gene containing } \\
\text { pCas for further mutagenesis }\end{array}$ & This study \\
\hline EHEC 86-24 pCas & $\begin{array}{l}\text { WT } 86-24 \text { carrier of pCas plasmid for } \\
\text { mutagenesis }\end{array}$ & This study \\
\hline DH5 $\alpha$ pTargetF $\Delta \operatorname{esp} A$ & Carrier of the espA gene targeting guide RNA & This study \\
\hline DH5 $\alpha$ pTargetF $\Delta e a e$ & Carrier of the eae gene targeting guide RNA & This study \\
\hline DH5 $\alpha$ pTargetF $\Delta l e r$ & Carrier of the ler gene targeting guide RNA & This study \\
\hline $\begin{array}{l}\text { EHEC 86-24_basR } \\
\text { pBAD-BasR }\end{array}$ & $\begin{array}{l}\text { Complemented the bas } R \text { gene back into the bas } R \\
\text { isogenic mutant on an arabinose inducible } \\
\text { promoter. }\end{array}$ & This study \\
\hline $\begin{array}{l}\text { EHEC 86-24_basS } \\
\text { pBAD-BasS }\end{array}$ & $\begin{array}{l}\text { Complemented the } b a s S \text { gene back into the bas } S \\
\text { isogenic mutant on an arabinose inducible } \\
\text { promoter. }\end{array}$ & This study \\
\hline $\begin{array}{l}\text { EHEC } \triangle e s p A \text { pCR2.1- } \\
\text { EspA-GFP }\end{array}$ & $\begin{array}{l}\text { The esp promoter controlling the transcription of } \\
\text { espA followed by a GFP gene reporter. }\end{array}$ & This study \\
\hline \multicolumn{3}{|l|}{ Plasmids } \\
\hline Plasmid & Description & Reference \\
\hline pCas & $\begin{array}{l}\text { Cas9 and } \lambda \text {-Red carrying plasmid with kanamycin } \\
\text { resistance }\end{array}$ & Sheng Yang \\
\hline pTargetF & $\begin{array}{l}\text { sgRNA plasmid with spectinomycin resistance to } \\
\text { direct Cas9 with the cadA guide }\end{array}$ & Sheng Yang \\
\hline pCR2.1-TOPO & Cloning vector & Thermo Fisher \\
\hline pTargetF $\Delta$ esp $A$ & $\begin{array}{l}\text { sgRNA plasmid to direct Cas9 with the esp } A \\
\text { guide }\end{array}$ & This study \\
\hline pTargetF $\Delta e a e$ & $\begin{array}{l}\text { sgRNA plasmid to direct Cas9 with the eae } \\
\text { guide }\end{array}$ & This study \\
\hline pTargetF $\Delta l e r$ & sgRNA plasmid to direct Cas9 with the ler guide & This study \\
\hline pCR2.1-EspA-GFP & $\begin{array}{l}\text { The esp promoter controlling the transcription of } \\
\text { esp } A \text { followed by a GFP gene reporter. }\end{array}$ & This study \\
\hline
\end{tabular}


All the bacterial strains were expanded on LB agar plates with appropriate antibiotics, kanamycin and spectinomycin for selection. The mutant clones were all preserved in $25 \%$ glycerol stocks in the $-80^{\circ} \mathrm{C}$ freezer. The pCas containing strains were grown at $30^{\circ} \mathrm{C}$ with kanamycin $(50 \mu \mathrm{g} / \mathrm{mL})$ selection. The pTargetF containing strains were grown at $37^{\circ} \mathrm{C}$ with spectinomycin $(50 \mu \mathrm{g} / \mathrm{mL})$ selection. The mutant strains were recovered on both kanamycin (50 $\mu \mathrm{g} / \mathrm{mL})$ and spectinomycin $(50 \mu \mathrm{g} / \mathrm{mL})$ double selection plates.

\subsection{Isogenic Mutant Construction Using CRISPR-Based Genome Editing}

The objective of this study was to generate isogenic mutants within EHEC O157:H7 strain 86-24 in genes that are associated with the T3SS. The mutants were screened through colony PCR method using size shift primers (Table II), where if the size of the gene product was that of the gene size, the mutagenesis was unsuccessful, while if the size of the gene product was less than the expected gene size, the mutagenesis was successful (Appendix A). Of the 38 clones tested, 5 showed a shift in size from the original esp $A$ gene size. This amplified product was yet again verified using 1\% agarose gel electrophoresis (Appendix A) with a portion of that PCR product being sent in for sequencing. Sequencing data validated that the CRISPR-Cas9 method generated a scar-free deletion in the EHEC 86-24 chromosome of gene espA.

The first round of mutagenesis tested two methods, one method used an 80 base pair ssDNA strand that contained 40 base pairs of sequence immediately before the beginning of the $\operatorname{esp} A$ gene and 40 base pairs immediately after the stop codon of the $\operatorname{esp} A$ gene. This method was meant to render a complete deletion of the esp $A$ gene region without the use of an antibiotic cassette.

The second method used, involved ordering an 80 base pair ssDNA sequence that was identical to the $\operatorname{esp} A$ gene region, that contained a stop codon shortly into the $\operatorname{esp} A$ gene region. 
The two approaches were used to test whether one method is more successful than the other. The result was that both methods were equally successful, due to this fact, the ler and double mutant $\Delta l e r \Delta \operatorname{esp} A$ mutagenesis involved a complete deletion rather than addition of a stop codon within the gene sequence in case a truncated version of the protein would affect the future results.

The method used to generate the isogenic mutants within the EHEC O157:H7 strain 8624 chromosome was the novel $\underline{\text { Clustered }} \underline{\text { Regularly }} \underline{\text { Interspaced }} \underline{\text { Palindromic }}$ Repeats (CRISPR)Cas9 method. This method was used to generate chromosomal deletions in the gene LEE4 encoded gene, espA, and the LEE1 encoded gene, and master regulator of the LEE PAI gene ler. The goal of generating the knock-out mutants within this EHEC strain was to observe the attachment behavior response of EHEC without the genes present. The genes esp $A$ and ler are well characterized as being essential to adherence and attachment to epithelial cells. ${ }^{173}$ The CRISPR-Cas9 mutagenesis was first attempted in one of two ways: (1) a stop codon was to be inserted within the gene sequence at the first available protospacer adjacent motif (PAM) sequence, (2) the gene of interest in its entirety would be removed from the chromosome.

The preliminary gene knock-out was undertaken on the gene esp $A$ where the two methods of mutagenesis were applied. Single stranded DNA (ssDNA) that was 80 base pairs (bp) in length were used to achieve the mutagenesis. One of the ssDNA contained the exact region 40 bp upstream of the PAM sequence and $40 \mathrm{bp}$ downstream of the PAM sequence, with three consecutive stop codons downstream of the PAM sequence. The other ssDNA primer selected a PAM sequence near the end of the esp $A$ gene region and consisted of $40 \mathrm{bp}$ upstream of the espA gene and $40 \mathrm{bp}$ downstream of the PAM sequence at the end of the espA gene. Both methods were tested, and both generated equivalent mutation efficiency. Due to this reason, the deletion method was undertaken as this method would leave a scar free deletion in the chromosome, thus, 
preventing any partial protein from being potentially produced. The same deletion method utilized on the $\operatorname{esp} A$ gene was undertaken on the ler gene, where a successful deletion of ler was completed. Lastly, because the strain EHEC 86-24\espA containing pCas existed from the first mutagenesis experiment, a second round of mutagenesis was conducted on that mutant strain. This entailed transforming the bacteria with the targeting plasmid pTarget $\Delta l e r$ and the ler specific ssDNA to create a double mutant $86-24 \Delta e s p A \Delta l e r$. Double mutations are especially laborious to conceive using the traditional mutagenesis protocols.

CRISPR/Cas9 mediated mutagenesis, gene knockout, and gene insertion in enterohemorrhagic Escherichia coli O157:H7 (EHEC 86-24) was based on the sequenced genome of EHEC EDL933 (AE005174.2). The use of ssDNA as a repair template instead of dsDNA or plasmid was elicited in the protocol. ${ }^{174}$

The materials necessary for this experiment included pCas plasmid (Addgene, Plasmid \#62225) and pTargetF plasmid (Addgene, Plasmid \#62226). Additionally, it was necessary to design 20 base pair sgRNA (site guide RNA) primers to use with the Q5 ${ }^{\circledR}$ Site Directed Mutagenesis Kit in order to replace the pTargetF RNA guide to the desired sequence. The pTargetF plasmid had a placeholder guide RNA sequence for the gene cadA. Primers were designed to replace cadA for the genes of interest to this study; espA, eae, and ler. The antibiotic resistance of pCas was Kanamycin and pTargetF was Spectinomycin.

DH5 $\alpha$ bacteria containing pCas were grown at $30^{\circ} \mathrm{C}$ shaking overnight for plasmid extraction the following day. The plasmid was extracted using the Geneaid High Speed Plasmid Mini Kit. Once the plasmid was extracted from DH5 $\alpha$, the 10,800 bp plasmid was electroporated into EHEC 86-24. 


\subsubsection{EHEC Electroporation}

EHEC 86-24 was inoculated overnight in $5 \mathrm{ml}$ of LB. The overnight was subcultured at a minimum of 1:50 into fresh LB media. This subculture was allowed to grow to $\mathrm{OD}_{600}$ of 0.4 to 0.6 at which point it was placed on ice. The culture was allowed to chill, at which point $20 \mathrm{ml}$ of culture was transferred to a $50 \mathrm{ml}$ Falcon tube and pelleted at 3000xg for 15 minutes. The supernatant was discarded, and the pellet was washed in $1 \mathrm{ml}$ of ice-cold $20 \%$ glycerol - $1 \mathrm{mM}$ MOPS (unbuffered), transferred to a $1.5 \mathrm{ml}$ sterile Eppendorf tube, and spun in a microfuge for 30 seconds (moderate speed). The supernatant was removed; the cells were resuspended in $1 \mathrm{ml}$ of the same buffer and re-centrifuged. Electroporation cuvettes (BioRad/BulldogBio) were cooled in an ice-water bath for at least 10 minutes prior to use. DNA samples contained either $0.1-0.5 \mu \mathrm{g}$ of purified DNA fragments or $0.2-10.0 \mu \mathrm{g}$ of plasmid digests in TE or water. A 50 $\mu \mathrm{l}$ sample of cells was mixed with $1-5 \mu \mathrm{l}$ of DNA, transferred to the electroporation cuvette, and incubated on ice for 1 minute. The cuvette was thoroughly but quickly dried and the cells were shocked as described previously [2]. Following electroporation, the cells were recovered by suspension in $2 \mathrm{ml} \mathrm{LB}$, shaking at $30^{\circ}$ for 1.5 hour, and plated on LB plates containing $50 \mu \mathrm{g} / \mathrm{ml}$ kanamycin.

\subsubsection{Q5 Site Directed Mutagenesis Kit Protocol}

Substitutions were created by designing a mismatch in the center of the mutagenic primer. At least 10 nucleotides were included that are complementary to the pTarget plasmid at the $3^{\prime}$ end of the primer. The $5^{\prime}$ end of the second primer began at the base next to the $5^{\prime}$ end of the first primer and proceeded in the opposite direction on the complementary strand. The second primer contained mismatches. The absence of any overlap ensures that exponential (rather than 
linear) amplification will take place. The maximum size of the substitution is largely dictated by oligo synthesis limitations.

\subsubsection{Exponential Amplification (PCR)}

The following reagents were assembled in a thin-walled PCR tube; 1X Q5 Hot Start High-Fidelity $2 \mathrm{X}, 0.5 \mu \mathrm{M}$ of $10 \mu \mathrm{M}$ forward primer, $0.5 \mu \mathrm{M}$ of $10 \mu \mathrm{M}$ reverse primer, $1-25 \mathrm{ng}$ of template DNA (1-25 ng/ $\mu 1)$, and $9.0 \mu 1$ of nuclease-free water. The reagents were mixed and transferred to a thermocycler with the following conditions: initial denaturation at $98^{\circ} \mathrm{C}$ for 30 seconds, 25 cycles at $98^{\circ} \mathrm{C}$ for 10 seconds followed by $50-72^{\circ} \mathrm{C}$ for $10-30$ seconds, then $72^{\circ} \mathrm{C}$ for 20-30 seconds $/ \mathrm{kb}$, final extension at $72^{\circ} \mathrm{C}$ for 2 minutes and lastly held at $4-12^{\circ} \mathrm{C}$.

\subsubsection{Kinase-Ligase-DpnI (KLD) Reaction}

The KLD reaction required the assembly of $1 \mu 1$ of PCR product with $5 \mu 1$ of $2 \mathrm{X}$ KLD Reaction Buffer, $1 \mu$ l of 10X KLD Enzyme Mix, and $3 \mu 1$ of nuclease-free water. The reaction was incubated for 5 minutes at room temperature after which it was transformed into chemically competent DH5 $\alpha$ cells. This was done by adding 1:5 ratio of KLD reaction products to chemically-competent DH5 $\alpha$. Allowing the cells to incubate on ice for 30 minutes, heatshocking the cells at $42^{\circ} \mathrm{C}$ for 30 seconds, then incubating on ice for 5 minutes. The heat shocked cells received a ratio of $1: 20$ cell to SOC media and allowed to recover at $37^{\circ} \mathrm{C}$ for 1 hour with shaking. The recovered cells were spread plated at lower and higher volumes on spectinomycin $(50 \mu \mathrm{g} / \mathrm{ml})$ and incubated overnight at $37^{\circ} \mathrm{C}$. The colonies were tested the next day for the presence of the pTargetF (Table III). Once the presence of the pTargetF was confirmed, the plasmid was sent for sequencing for the confirmation of the inserted RNA guide to TCAG Facilities at The Centre for Applied Genomics. 


\subsubsection{Gene Replacement Protocol}

Once EHEC 86-24 was confirmed to contain pCas, the single stranded DNA primers were designed as deletions, and pTargetF was confirmed to contain the guide RNA for the genes; espA, eae, and ler the mutagenesis reaction was performed. This reaction was similar to the electroporation reaction where the $86-24$ pCas was grown overnight at $30^{\circ} \mathrm{C}$ and subcultured at minimum 1:50 with kanamycin and allowed to reach $\mathrm{OD}_{600}$ of 0.6 or at a concentration of $\sim 10^{8}$ cells $/ \mathrm{ml}$ at $30^{\circ} \mathrm{C}$. Once the subculture reached $\mathrm{OD}_{600}$ of $0.6\left(\sim 10^{8}\right.$ cells $\left./ \mathrm{ml}\right)$, induction of $\lambda$-Red protein expression was induced with $50 \mathrm{mM}$ final concentration of L-Arabinose for 20 minutes at $30^{\circ} \mathrm{C}$ and 250 r.p.m. The recombinase produced during the $\log$ growth to $\mathrm{OD}_{600}$ of 0.6 was sufficient to ensure recombination. Longer induction time results in cell death from toxicity of the Gam protein. The cells were ready when the $\mathrm{OD}_{600}$ is $0.4-0.6$ yet it was important not to overgrow cells as stationary phase cells are not optimal for recombineering.

Immediately after induction the culture was rapidly chilled in an ice-water slurry for 5 minutes by swirling. From this point the cultures were kept ice cold as keeping the cells at a low temperature until electroporation prevented the breakdown of recombination proteins and maintained a high allelic recombination frequency. All subsequent steps were performed at 0 $2^{\circ} \mathrm{C}$ on wet ice.

The induced culture was made electrocompetent by the method outlined in 2.1.1. Once the electrocompetent cells were prepared, the cells were combined with the plasmid and single stranded (ssDNA) in pre-chilled Eppendorf tubes. This involved combining 50 $\mu 1$ of EHEC 86-24 pCas containing cells with $1 \mu \mathrm{l}$ (100ng) of pTarget-sgRNA and $1 \mathrm{ul}$ (10 $\mu \mathrm{M}$ final) of the ssDNA oligonucleotide (Table II). The control for this experiment was 86-24 pCas with pTarget-sgRNA 
with no ssDNA. This control was expected to generate no colonies as the Cas 9 enzyme was expected to create a double stranded break in the DNA of the bacteria, killing the organism.

The cells, plasmid and ssDNA were transferred to an ice-cold cuvette. Electroporation was performed on a BioRad Gene Pulser to deliver $18 \mathrm{kV} / \mathrm{cm}, 200 \mathrm{ohms}$ and $25 \mu \mathrm{F}$ for a $1 \mathrm{~mm}$ cuvette, with a time constant approximating 5 milliseconds. Cells were immediately recovered in $1 \mathrm{ml}$ final volume of SOC media with $50 \mu \mathrm{g} / \mathrm{mL}$ kanamycin for 2 hours at $30^{\circ} \mathrm{C}$ before plating $20 \mu \mathrm{l}$ and $200 \mu \mathrm{l}$ on plates containing $50 \mu \mathrm{g} / \mathrm{mL}$.

Table II: Primer Sequences for CRIPSR Gene Editing

\begin{tabular}{|l|l|}
\hline Gene Target & Primers \\
\hline espA Q5 sgRNA Guide Forward & AATATGTCGAGTTTTAGAGCTAGAAATAGC \\
\hline espA Q5 sgRNA Guide Reverse & ACCTAAGTCAACTAGTATTATACCTAGGAC \\
\hline espA Delete Oligo 5' & $\begin{array}{l}\text { TTATTTACCAAGGGATATTGCTGAAATAGTTCTATA } \\
\text { CACATTAACAACGGATGTTGCATTTGATGTATCCAT }\end{array}$ \\
\hline ler Q5 sgRNA Guide Forward & TACAAGAGCTGTTTTAGAGCTAGAAATAGC \\
\hline ler Q5 sgRNA Guide Reverse & AGGTTATATTACTAGTATTATACCTAGGAC \\
\hline Ler Delete Oligo 5 & $\begin{array}{l}\text { TTAAATATTTTTCAGCGGTATTATTTCTTCTTCAG } \\
\text { TATTATTTTCCATATTCATAATAAATAATCTCCGCAT }\end{array}$ \\
\hline
\end{tabular}

The 80 bp ssDNA template oligos for recombination with two $5^{\prime}$ thiophosphate bonds (Sigma) while the 50 bp primers were used in the site directed mutagenesis (SDM) of the pTarget-F plasmid.

\subsubsection{Colony PCR Genotyping}

Transformants were genotyped by allele specific PCR. For mutations/insertions: the 3' end of the mutant genotyping primer annealed perfectly to the mutant genotype, while wild-type possessed mismatched DNA at the $3^{\prime}$ end. Hotstart Taq polymerase that does not possess $3^{\prime}$ to $5^{\prime}$ exonuclease activity is used for colony PCR of the putative mutants. Only those cells which had 
incorporated the mutation produced PCR product since the mismatch between the $3^{\prime}$ end of the primer and wild-type genome does not allow for primer extension.

The deletions were screened by using PCR primers up and downstream of the deletion. A shift in PCR product size was observed by DNA gel electrophoresis. Deleted gene PCR products were smaller than that of WT gene.

\subsection{7 pTargetF Plasmid Curing}

The curing of pTarget series was performed on the edited colony harboring both $\mathrm{pCas}$ and pTarget series is inoculated into $2 \mathrm{ml}$ of LB medium containing kanamycin $(50 \mu \mathrm{g} / \mathrm{mL})$ and IPTG (isopropyl-D-thiogalactopyranoside; $0.5 \mathrm{mM}$ final). The culture was incubated at $30^{\circ} \mathrm{C}$ for $8 \mathrm{~h}$ to $16 \mathrm{~h}$. Diluted culture was spread onto LB plates containing only kanamycin (50 $\mu \mathrm{g} / \mathrm{ml})$. The colonies are confirmed as cured by determining their sensitivity to spectinomycin (50 $\mu \mathrm{g} / \mathrm{ml})$. The colonies cured of $\mathrm{p}$ Target series were used in a second round of genome editing or cured of pCas to get the final strain.

\subsection{8 pCas Plasmid Curing}

The pCas plasmind contains a temperature-sensitive replicon to allow for its easy elimination. pCas was cured by growing the colonies overnight at $37^{\circ} \mathrm{C}$, non-selectively. 


\section{Table III: Primers used in CRISPR/Cas9 for $\operatorname{esp} A$ Gene Mutagenesis}

\begin{tabular}{|c|c|c|c|c|c|}
\hline \multicolumn{6}{|l|}{ Primers } \\
\hline Name & Sequence (5'-3') & $\begin{array}{l}\text { Length } \\
\text { (bp) }\end{array}$ & $\% \mathrm{GC}$ & $\mathrm{Tm}$ & $\mathrm{Ta}^{*}$ \\
\hline Q5_SDM_sgRNA_espA_F & aatatgtcgaGTTTTAGAGCTAGAAATAGC & 30 & $33 \%$ & $56^{\circ} \mathrm{C}$ & \multirow[t]{2}{*}{$57^{\circ} \mathrm{C}$} \\
\hline Q5_SDM_sgRNA_espA_R & acctaagtcaACTAGTATTATACCTAGGAC & 30 & $37 \%$ & $56^{\circ} \mathrm{C}$ & \\
\hline espA_deletion_oligo $(+)$ & $\begin{array}{l}\mathrm{t} * \mathrm{t} * \text { atttaccaagggatattgctgaaatagttctatacacattaacaa } \\
\text { cggatgttgcatttgatgtatccat }\end{array}$ & 72 & $31.94 \%$ & $\begin{array}{l}67.21^{\circ} \\
\mathrm{C}\end{array}$ & N/A \\
\hline espA_STOP_oligo $(+)$ & $\begin{array}{l}\text { g*a*aaaacaccgagttcctcaaatagcttaaccacctcatcTC } \\
\text { ATTAcatattacctaagtcatagatcgtcgatgtcgaagaactc }\end{array}$ & 86 & $39.53 \%$ & $69.14 \%$ & N/A \\
\hline $\begin{array}{l}\text { espA_Mut/Del_colony } \\
\text { PCR_F }\end{array}$ & aatagccgecttcactgtttgc & 22 & $50 \%$ & $58.2^{\circ} \mathrm{C}$ & \multirow[t]{2}{*}{$55^{\circ} \mathrm{C}$} \\
\hline $\begin{array}{l}\text { espA_Mut/Del_colony_PC } \\
\mathrm{R} \text { R }\end{array}$ & ctatgacttaggtaatatgtcgaag & 25 & $36 \%$ & $\begin{array}{l}50.65^{\circ} \\
\mathrm{C}\end{array}$ & \\
\hline espA_Delete_Size_Shift_F & aaccccagacgttacagacagg & 22 & $54.55 \%$ & $\begin{array}{l}58.04^{\circ} \\
\mathrm{C}\end{array}$ & \multirow[t]{2}{*}{$55^{\circ} \mathrm{C}$} \\
\hline espA_Delete_Size_Shift_R & agtgagcagagagagaatgcat & 22 & $45.45 \%$ & $\begin{array}{l}55.34^{\circ} \\
\mathrm{C}\end{array}$ & \\
\hline
\end{tabular}

* Ta (recommended annealing temperature)

The primers that were used in the generation of the EHEC 86-24 $\triangle$ esp $A$ were designed to optimally be incorporated into the bacterial genome. The stars for the deletion and stop oligos are the two thiophosphate bonds at the 5 ' region to prevent oligonucleotide degradation by endogenous enzyme. 


\subsection{Western Blot}

Two sets of glass plates as well as two combs (1.5mm thickness glass and combs) were washed and dried. Gel pouring stand was assembled with extra care to make sure the glasses were level to prevent leakage. The reagents for the separating gel were combined in a labelled tube - APS or TEMED were not added until the gel was ready to pour. The reagents for a $10 \%$ gel include $12 \mathrm{~mL} \mathrm{H}_{2} \mathrm{O}, 10 \mathrm{~mL} \mathrm{30 \%}$ acrylamide (in $4 \mathrm{C}$ ), $7.5 \mathrm{~mL}$ resolving buffer (1.5M Tris, $\mathrm{pH}$ 8.8), $300 \mathrm{uL} \mathrm{10 \%} \mathrm{SDS,} \mathrm{100uL} \mathrm{10 \%} \mathrm{APS,} \mathrm{and} 28 \mathrm{uL}$ TEMED. A transfer pipette was used to add resolving gel mixture between plates while leaving enough space to add stacking gels. A layer of $95 \%$ ethanol (or methanol) was added to the top of the resolving gel to remove bubbles and ensure a smooth top to the layer. After the leftover resolving gel has polymerized in the tube, ethanol was poured off and wash 3 times with $\mathrm{ddH}_{2} \mathrm{O}$. Following the solidification of the separating gel, the resolving gel reagents were assembled. The reagents for the resolving gel were combined; $5.7 \mathrm{~mL} \mathrm{H} \mathrm{H}_{2} \mathrm{O}, 1.65 \mathrm{~mL} 30 \%$ acrylamide (in $\left.4^{\circ} \mathrm{C}\right), 2.5 \mathrm{~mL}$ resolving buffer $(1.5 \mathrm{M}$ Tris, pH 8.8), $100 \mathrm{uL} \mathrm{10 \%} \mathrm{SDS,} 60 \mathrm{uL} \mathrm{10 \%} \mathrm{APS,} \mathrm{and} 16 \mathrm{uL}$ TEMED. A new transfer pipette was used to add stacking gel between plates, the gel fill to the top of the plates. The $1.5 \mathrm{~mm}$ comb was placed in between the two plate. Once the stacking gel polymerized in the tube, the gels were removed from the pour stand and used in the experiment or wrapped in damp paper towels and stored in at $4^{\circ} \mathrm{C}$ for no longer than 3 days.

\subsubsection{Bacterial culture preparation}

The bacteria were stressed with either $30 \mathrm{mM}$ or $172 \mathrm{mM}$ short chain fatty acid mixes by sub culturing with LB, MOPS, in a shaking incubator until OD 1.00. Once the desired OD was reached, the samples were spun down at $3500 \mathrm{rpm}$ for 10 minutes to pellet the bacteria. The 
supernatant was discarded into waste container and the pellet was either stored at $-80^{\circ} \mathrm{C}$ or used in the sample preparation step.

\subsubsection{Sample preparation}

Excess LB was removed from pellet and the pellet was washed twice with PBS (1X). The washed pellet was re-suspended in 1X SDS loading buffer (4x: 4mL Glycerol (100\%), 4.8mL 0.5M Tris $\mathrm{HCl}$ (pH 6.8), 0.8g SDS, 4mg Bromophenol blue, 0.5mL B-mercaptoethanol, 0.7mL $\mathrm{H}_{2} \mathrm{O}$ ). The sample was boiled for 10 minutes at $95^{\circ} \mathrm{C}$ and a 27 -gage needle with $1 \mathrm{~mL}$ syringe was used to shear DNA and cellular structures. The samples can be stored in $-20^{\circ} \mathrm{C}$ until ready for use.

\subsubsection{SDS-PAGE}

The cell lysates were pelleted in the microcentrifuge for 10 minutes at $12,000 \mathrm{rpm}$ to spin down debris. Appropriate volumes of each sample were loaded such that all lanes contain the same amount of protein. FroggaBio BLUelf Prestained Protein ladder was used at a volume of $5 \mu l$. The gel was run for 45 minutes at $70 \mathrm{~V}$ (constant voltage) to move the proteins through the stacking gel. It was made sure that all samples go through the stacking gel before increasing the voltage for the resolving gel. The voltage was then changed to $120 \mathrm{~V}$ and the gel was allowed to run for 2 hours 20 minutes until the tracking dye has begun to leak out into the outer chamber.

\subsubsection{Transfer}

Immobilon-P PVDF Membrane by Millipore was used for the transfer of proteins. Two blotting papers were placed outside of each PVDF membrane and gel. The membrane should be slightly larger than the SDS-PAGE gel, and the blotting papers a bit larger than the membrane. The PVDF membrane was activated in methanol, then rinsed with $\mathrm{ddH}_{2} \mathrm{O}$. The transfer cassettes 
were assembled and placed in the tank with cold transfer buffer. An ice pack was also placed in the apparatus. The transfer was run for 2 hours at minimum $100 \mathrm{~V}$ (constant).

After 2 hours, the cassette was disassembled and the PVDF containing protein was placed in blocking buffer (5\% BSA suspended in PBS) for 1 hour. Once blocked, the membrane was incubated in $1^{\circ}$ antibody ( $\alpha$-EspA or $\alpha$-Intimin $+5 \%$ BSA) overnight at $4^{\circ} \mathrm{C}$. After o/n incubation with primary antibodies, the membrane was washed with 1x TBST (3 quick washes followed by 30 min washes, 3 times with shaking). The membrane was transferred to clean square plates then the diluted secondary antibodies was added to the container. The primary antibodies were all anti-mouse and therefore the same secondary antibodies could be used for each of the primary antibodies for: anti-intimin, anti-EspA, and anti-DnaK. The blot was incubated with the secondary antibodies for 1 hour at room temperature. After incubation with secondary antibodies, the membrane was washed with 1x TBST (3 quick washes followed by 30 min washes, 3 times with shaking).

\subsubsection{Detection}

The proteins were detected using the Millipore Luminata ${ }^{\mathrm{TM}}$ Crescendo and Classico Western HRP Substrate. Each PVDF membrane received $1 \mathrm{ml}$ of the HRP Substrate and allowed to incubate. The Western blot was imaged using a BioRad ChemiDoc gel imaging system. 


\subsection{GFP Reporter Assay}

EHEC O157:H7 strain 86-24_esp $A+$ pCR2.1-esp $A$-GFP was grown O/N. The optical density $\left(\mathrm{OD}_{600}\right)$ was normalized to 0.05 in the following manner. The $\mathrm{O} / \mathrm{N}$ culture $(1 \mathrm{~mL})$ was transferred to an Eppendorf tube and washed twice in HEPES buffer (10 mM; pH 7.2). After last re-suspension in HEPES (10 mM; $\mathrm{pH} 7.2)$, a 1:10 dilution was done in order to measure the $\mathrm{OD}_{600}$. The volume of the diluted $\mathrm{O} / \mathrm{N}$ culture needed in each well was calculated using $\mathrm{C}_{1} \mathrm{~V}_{1}=\mathrm{C}_{2} \mathrm{~V}_{2}$ where each well will have $225 \mu \mathrm{l}\left(\mathrm{V}_{2}\right)$ of media.

The plate set up involved the use of Costar Black 96-well plates with clear bottom from Corning (Corning, New York, United States). A volume of $250 \mu \mathrm{L}$ of $\mathrm{H}_{2} \mathrm{O}$ was added to all the outside wells to avoid evaporation of the samples. The interior wells were used for all the strains, technical replicates and controls. Media containing all the treatments and appropriate antibiotics (kanamycin $50 \mu \mathrm{g} / \mathrm{mL}$ ) were added to each set of wells first, using the volumes calculated. One set of wells for each biological replicate possessed just $225 \mu \mathrm{L}$ of media for the control.

The plate reader used was the BioTek Instruments, Inc. (Winooski, Vermont, United States) Synergy $^{\mathrm{TM}}$ HTX Multi-Mode Microplate Reader for fluorescence, absorbance and luminescence. The program set-up was as follows: excitation was set to $480 \mathrm{~nm}$ and emission is $528 \mathrm{~nm}$ (using the Excitation Wheel 1 and Emission Wheel 1 respectively). The temperature was set to $37^{\circ} \mathrm{C}$, and the plate was continuously shaken (orbital) for the duration of the experiment. The kinetic experiment lasted 16.5 hours, with reads of $\mathrm{OD}_{600}$ and fluorescence (taken from the bottom of the wells) every 30 mins, with the lid on the plate to prevent evaporation. Prior to the kinetic run, the plate was shaken for 15 seconds, following this an $\mathrm{OD}_{600}$ and fluorescence reading was taken for the time 0 points. The data was recorded by the Gen5 software, from which the data was exported in an excel file. The plate reader automatically subtracted the media control $\mathrm{OD}_{600}$ and 
fluorescence from all the experimental wells. The corrected fluorescence values were divided by the corrected $\mathrm{OD}_{600}$ values, and the technical and biological replicates were averaged to generate the final expression values for the esp promoter.

\subsection{Attachment Assay}

The HEp-2 cells were seeded into a 12 - well plate containing DMEM $+10 \%$ FBS $+1 \%$ PenStrep (100 U/mL Penicilium and $100 \mu \mathrm{g} / \mathrm{mL}$ Streptomycin) at the concentration of $1.0 \times 10^{5}$ cells per well as determined by a count using the hematocytometer. The HEp-2 cells were allowed to attach overnight at $37^{\circ} \mathrm{C}$ in $5 \% \mathrm{CO}_{2}$. Simultaneously, an overnight of the WT bacterial culture, and various mutant strains $(\triangle \operatorname{esp} A, \Delta l e r, \Delta e s p A \Delta l e r)$ were cultured.

On day two of the experiment, the $\mathrm{O} / \mathrm{N}$ bacterial cultures were subcultured 1:50 and allowed to grow until $\mathrm{OD}_{600}$ was approximately $0.4-0.5$. The bacterial cells were normalized to the same concentration $\left(\mathrm{OD}_{600}\right.$ of 0.4$)$ and plated in dilutions from $10^{-1}$ to $10^{-6}$ to generate an initial count. While the bacterial cells were growing in the subculture, the HEp-2 cells were aspirated of the antibiotic containing media, washed three times in PBS and fresh media free of antibiotics ( DMEM $+10 \% \mathrm{FBS}$ ) and warmed to $37^{\circ} \mathrm{C}$ was added to the wells. The HEp-2 cells were infected with a 100:1 MOI of bacteria to mammalian cells. The HEp-2 cells were infected for 3 hours at $37^{\circ} \mathrm{C}, 5 \% \mathrm{CO}_{2}$. The media was removed, and the cells were washed, and allowed another hour of infection in fresh media to a total of 4 hours of infection.

After the infection the media was removed, and the cells were washed 3 times in warm PBS. The cells were extracted using $0.1 \%$ Triton-X100 in two volumes and the contents of each well were diluted and plated in the various dilutions. Each of the dilutions were counted for the number of colonies grown and the $\mathrm{CFU} / \mathrm{mL}$ was calculated. The WT strain grown in $\mathrm{LB}$ was used as the $100 \%$ attachment strain, while the rest were calculated relative to the WT LB strain. 


\subsection{RESULTS}

\subsection{Isogenic Mutants Showed No Change in Growth Capacity Relative to Wild Type}

To ensure that the isogenic mutants behave the same as the unaffected EHEC 86-24, it was imperative to generate a growth curve. The growth curves were conducted in 96 well plates, with multiple replicates to ensure that the data was reliable. The bacteria were grown in low glucose (LG) DMEM as this is the media to be used in the subsequent experiments. Based on the data, it was evident that the deletion of espA, ler, and both espA and ler did not affect the growth of the bacteria when compared with the wild type (Figure 5). This was good evidence to suggest that the attachment of these bacteria can be tested to observe the differences in attachment that can be attributed to the bacteria lacking genes without their growth patterns being attributed to these changes in attachment. 


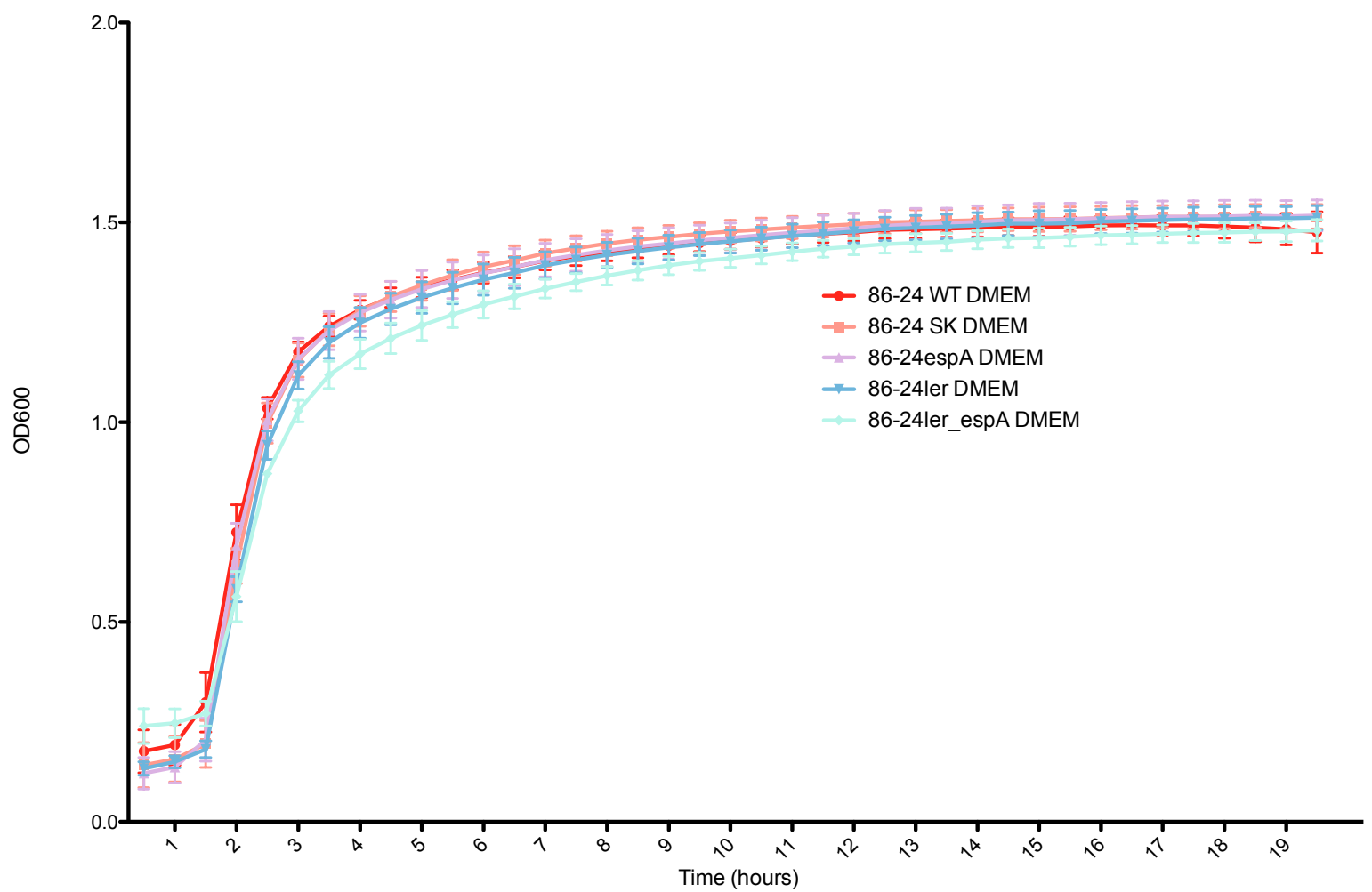

Figure 5. Deletion of $\operatorname{esp} A$, ler, or $\operatorname{esp} A$ and $l e r \operatorname{does}$ not affect EHEC growth

The growth curves of the various strains were conducted over a period of 19 hours. The growth was verified in DMEM as this is the media that will be used to grow the cell lines, and thus, where the infection would occur. The strains tested were EHEC 86-24 WT, 86-

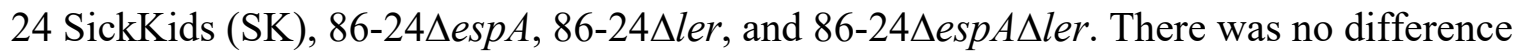
in growth between any of the strains tested. For this reason, it can be concluded, that any changes in attachment can be attributed to the bacterial virulence. 


\subsection{Attachment of EHEC Affected by SCFA and espA Mutation}

EHEC attachment was analyzed on Caco-2 cells as well as HEp-2 cells. The methods can be viewed in the Materials and Methods section. The difference among the various growth conditions and how the various conditions affect attachment are outlined below in Figure 6 and Figure 7.

\section{WT}
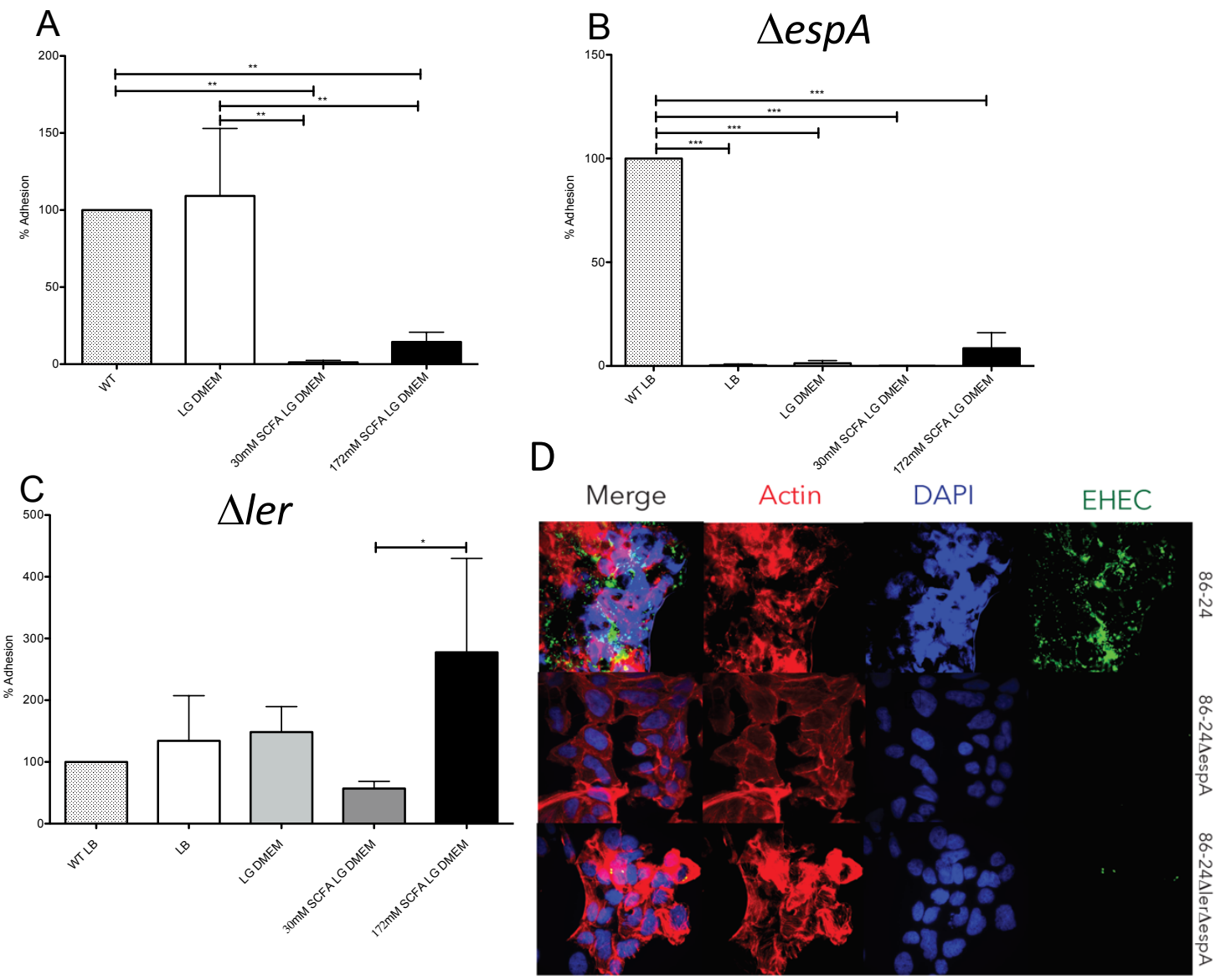

Figure 6. Attachment of EHEC 0157:H7 under various environmental conditions

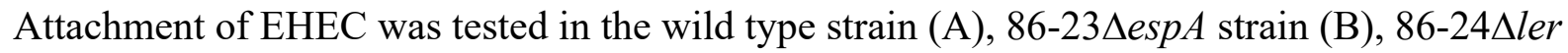
strain (C). Caco-2 cells were infected with WT EHEC and two mutant strains. Microscopy imaging showed a decrease in attachment for the esp $A$ mutant strain. Significance between the environmental conditions was observed in the wild type strain alone. This data is generated from 3 biological replicates and 2 technical replicates. (D) Infection of Caco-2 cells with 86-24 and 
various mutants as visualized via immunofluorescence. Data in panels A, B and C are presented as means \pm s.d.; $n=3 .{ }^{*} P \leq 0.05 ;{ }^{* *} P \leq 0.01 ;{ }^{* * *} P \leq 0.001$. All $P$ values were calculated using Tukey’s Multiple Comparison Test.

The wild type EHEC O157:H7 strain 86-24 attachment was calculated to be $100 \%$ when the bacteria were grown under the Luria Bertani (LB) condition and this strain acted as the control strain for all the subsequent experiments. In the WT strain, the attachment did not change under the low glucose (LG) Dulbecco's Modified Eagle's Medium (DMEM) condition where the $\%$ attachment was $109.2 \%$ (Figure $6-\mathrm{A}$ ). The attachment decreased significantly 83 -fold, to $1.2 \%$ for the $30 \mathrm{mM}$ short chain fatty acid (SCFA) treatment (Figure $6-\mathrm{A}$ ). Under the $172 \mathrm{mM}$ SCFA treatment the attachment decreased 7-fold compared to WT LB control (Figure $6-\mathrm{A}$ ).

The EHEC O157:H7 strain 86-24 2 esp $A$ exhibited a different trend where attachment in LB was very low at $0.35 \%$, significantly lower (286-fold) compared to WT LB control (Figure 6 - B). Growing the bacteria in LG DMEM did not have an effect on the attachment of 86$24 \Delta \operatorname{esp} A$ as it was $1.3 \%$ which was significantly lower (77-fold) than the WT LB control

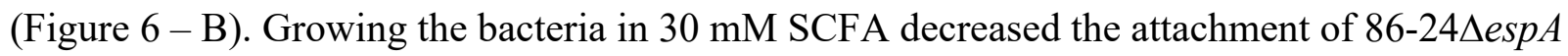
to $0 \%$ (Figure $6-\mathrm{B}$ ). The bacterial attachment in $172 \mathrm{mM} \mathrm{SCFA}$ of $86-24 \Delta \operatorname{esp} A$ was $8.5 \%$, significantly lower (11.8-fold) than the WT LB control (Figure $6-\mathrm{B})$.

The EHEC O157:H7 strain 86-24Aler did not have any significant change compared to the WT LB control. When 86-24Aler was grown in LB the attachment observed was $134.2 \%$, which was not statistically different from the WT control or any of the other conditions (Figure 6 $-\mathrm{C})$. This was a surprising result as the ler gene is highly associated with regulation of the LEE. Growing 86-24Aler in LG DMEM generated at a value of $148.3 \%$ attachment. Treatment of 8624Aler with $30 \mathrm{mM}$ SCFA generated an attachment of $56.9 \%$ which was significantly lower (5- 
fold) compared to the $172 \mathrm{mM}$ SCFA condition which yielded an attachment value of $277.7 \%$

(Figure 6-C).
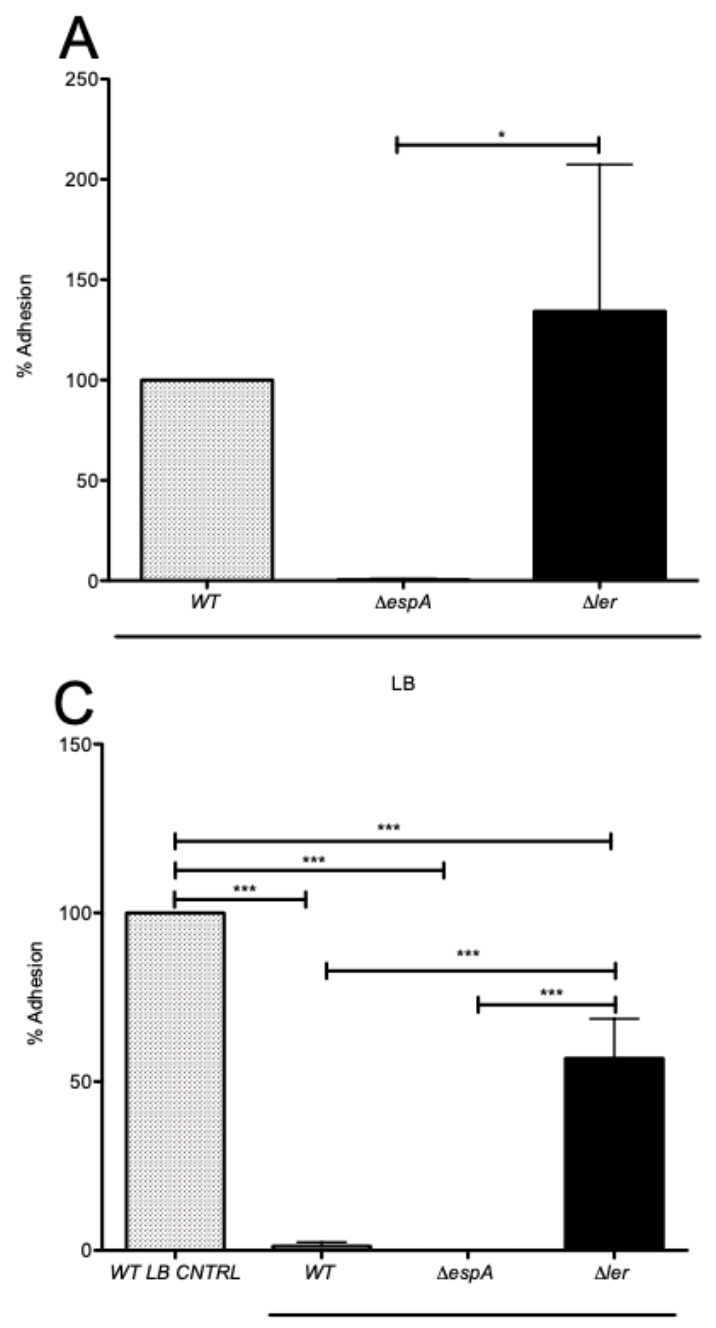

$30 \mathrm{mM}$ SCFA
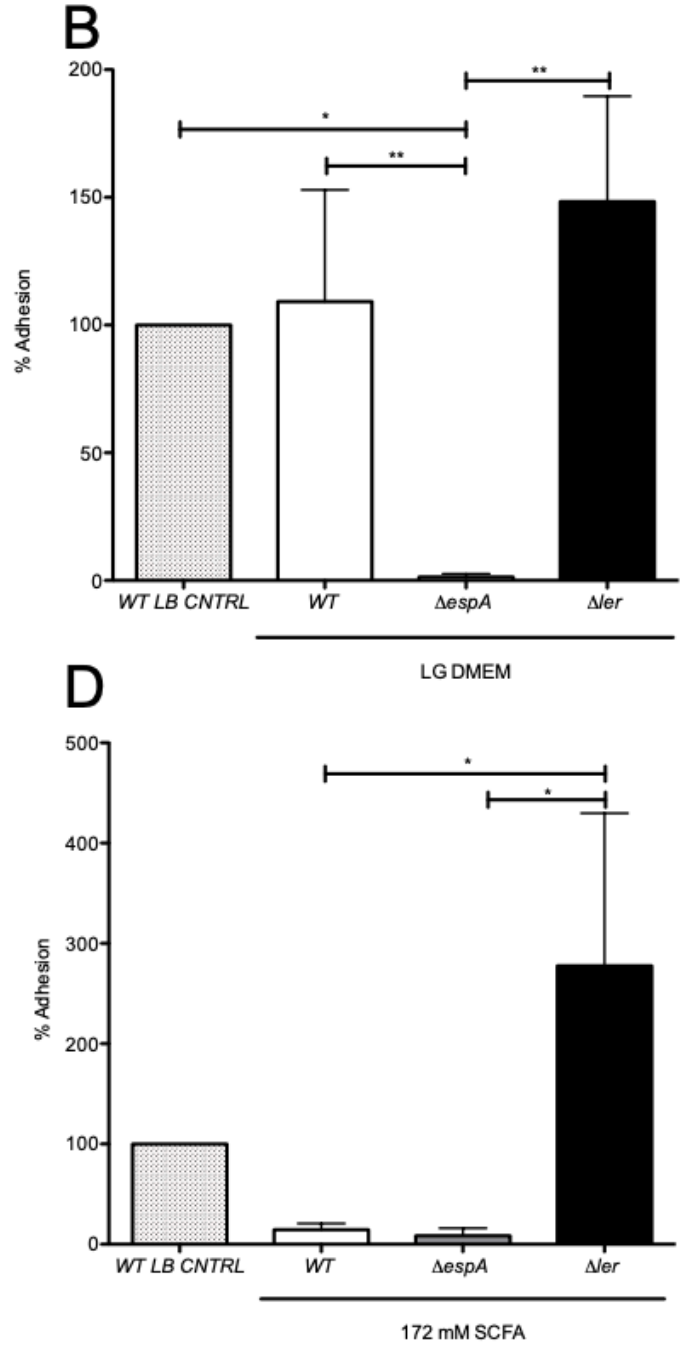

Figure 7. Attachment of various EHEC mutants compared

Attachment of EHEH O157:H7 was tested on HEp-2 cell line. The various conditions tested were as follows: LB (A), low glucose (LG) DMEM (B), LG DMEM with 30 mM SCFA (C), ${ }_{\text {tr }}$ LG DMEM with $172 \mathrm{mM}$ SCFA (D). Within each of the conditions, three different strains of EHEC 0157-H7 strain 86-24 were tested, wild type strain, espA knock-out mutant, and ler $\left(\mathrm{I}_{\mathrm{knock}-o u t}\right.$ mutant. This data is generated from 3 biological replicates and 2 technical wreplicates. Data are presented as means \pm s.d.; $n=3 .{ }^{*} P \leq 0.05 ;{ }^{* *} P \leq 0.01 ;{ }^{* * *} P \leq 0.001$. All $P$ (I values were calculated using Tukey's Multiple Comparison Test. 


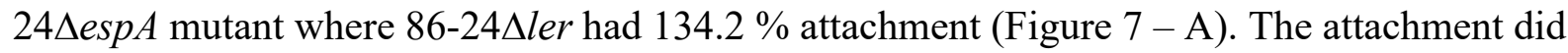
not differ from the wild type strain that $100 \%$ attachment compared to the $134.2 \%$ of $86-24 \Delta l e r$ (Figure $7-\mathrm{A}$ ).

The wild type strain attachment was at $109.1 \%$ in LG DMEM, the increase was not significant compared to the WT LB control. The WT LG DMEM and WT LB attachment were

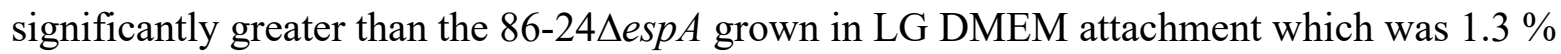

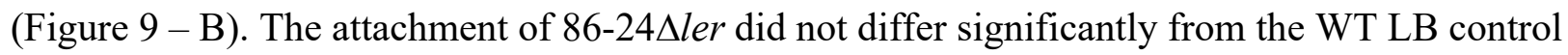
nor WT LG DMEM, it was however significantly higher than the $86-24 \Delta \operatorname{esp} A$ at $148.2 \%$ (Figure $7-\mathrm{B})$.

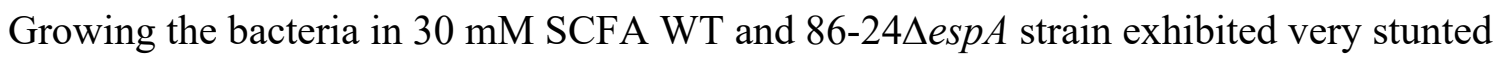

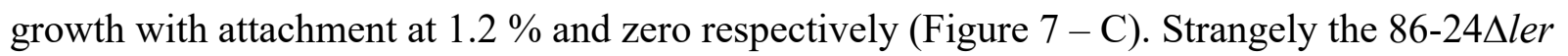
strain experienced robust growth with attachment values at $56.9 \%$, significantly (1.75-fold) lower than the WT LB control.

Growth of the various strains in $172 \mathrm{mM}$ SCFA generated a similar trend to the $30 \mathrm{mM}$

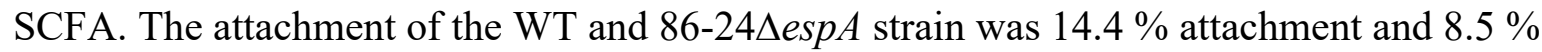

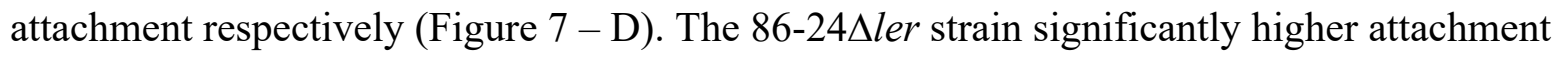

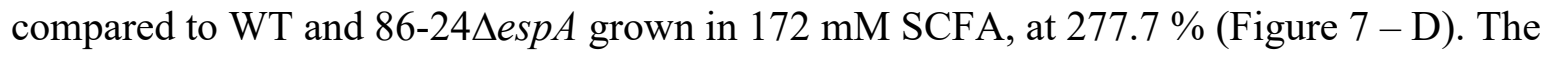
difference was not significant between 86-24Aler and the WT LB control (Figure 7 - D).

In summary, the attachment data resulted in some interesting findings with regard to the

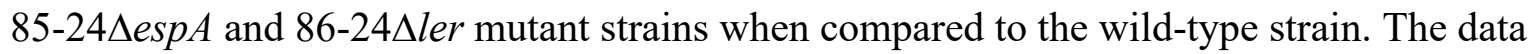
showed that removing the esp $A$ gene rendered the bacteria virtually incapable of attaching to HEp-2 epithelial cells, as well as Caco-2 cells. The inability for the EHEC to attach to epithelial cells was irrespective of treatment. Interestingly, the ler isogenic mutant showed similar 
attachment to EHEC wild type strain in all conditions except the SCFA treatment conditions. Remarkably, the ler mutant attachment did not suffer when it was grown in SCFA. In contrast, when the wild-type strain was grown in SCFA, the attachment was significantly diminished compared to LG DMEM and LB. This could suggest that that ler is exerting negative control on expression of adhesins elevated concentrations of SCFAs 


\subsection{Western Blot Analysis of T3SS Proteins}

The expression of EspA was analyzed in EHEC O157:H7 strain 86-24. This was done through western blotting with a 1:3000 dilution of anti-EspA and compared with the DnaK loading control that was used at a 1:10,000 dilution of anti-EspA. The raw data yielded by the Western Blotting experiment can be viewed in their raw form in the Appendix B. According to the data generated, it is evident that there are no statistically significant changes in EspA expression between the various treatment groups (Figure 8). In LG DMEM shaking, the ratio of EspA:DnaK relative to the LB control was 1.9. In the $30 \mathrm{mM}$ SCFA shaking condition, the ratio of EspA:DnaK relative to the $30 \mathrm{mM} \mathrm{NaCl}$ condition was 0.45 . In the $172 \mathrm{mM}$ SCFA shaking condition, the ratio of EspA:DnaK relative to $172 \mathrm{mM} \mathrm{NaCl}$ was 0.52 . In the $30 \mathrm{mM}$ SCFA to $172 \mathrm{mM}$ SCFA shaking condition, the ratio of EspA:DnaK relative to $30 \mathrm{mM} \mathrm{NaCL}$ to $172 \mathrm{mM}$ $\mathrm{NaCl}$ was 0.75 . Lastly, in the $30 \mathrm{mM}$ SCFA to $172 \mathrm{mM}$ SCFA static with mineral oil overlay, the ratio of EspA:DnaK relative to $30 \mathrm{mM} \mathrm{NaCl}$ to $172 \mathrm{mM} \mathrm{NaCl}$ static with mineral oil was 3.3. There was no significant difference between any of the treatment groups according to a OneWay ANOVA with Tukey's Multiple Comparison Test.

Together, this data suggests that the quantity of EspA protein remains unaffected regardless of the presence of SCFA or various media. This lack of significance, however, may be due to the integrity of the primary antibody used to prime for the presence of EspA protein. Therefore, further research should be done into exploring the abundance of EspA protein under the various conditions tested. This is important as the genetic data shows that the various treatments affect the activity of the esp promoter, that precedes the EspA gene. This means that there must be a change in the production of this protein. 

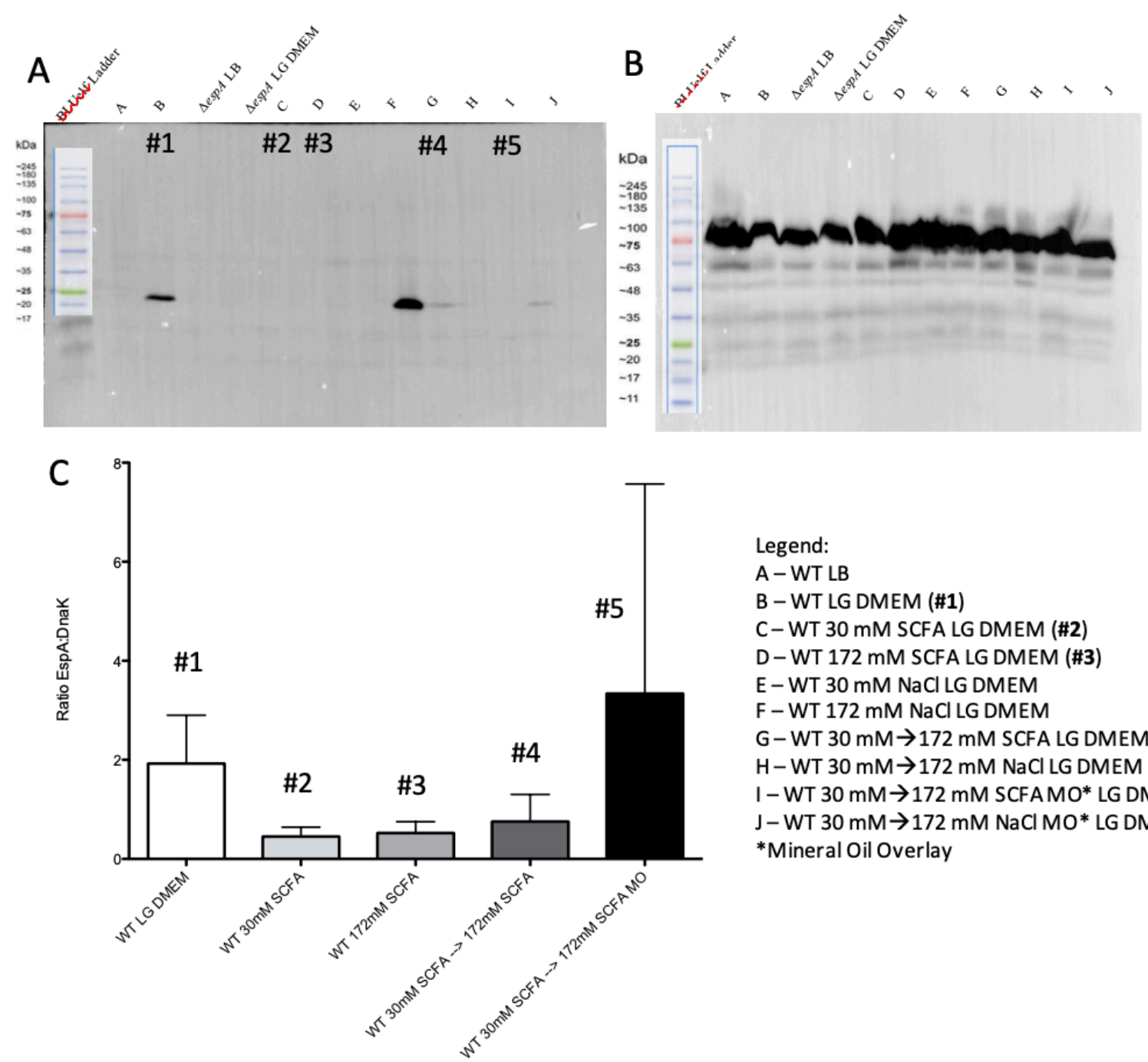

Legend

A - WT LB

B - WT LG DMEM (\#1)

C - WT 30 mM SCFA LG DMEM (\#2)

D - WT 172 mM SCFA LG DMEM (\#3)

E - WT $30 \mathrm{mM} \mathrm{NaCl} \mathrm{LG} \mathrm{DMEM}$

F - WT 172 mM NaCl LG DMEM

$\mathrm{G}-\mathrm{WT} 30 \mathrm{mM} \rightarrow 172 \mathrm{mM}$ SCFA LG DMEM (\#4)

$\mathrm{H}-\mathrm{WT} 30 \mathrm{mM} \rightarrow 172 \mathrm{mM}$ NaCl LG DMEM

I - WT $30 \mathrm{mM} \rightarrow 172 \mathrm{mM}$ SCFAMO* LG DMEM (\#5)

$\mathrm{J}-\mathrm{WT} 30 \mathrm{mM} \rightarrow 172 \mathrm{mM}$ NaCl MO* LG DMEM

*Mineral Oil Overlay

\section{Figure 8. Expression of EspA protein through western blotting}

This data was monitoring EspA protein expression using a monoclonal EspA antibody. A) The raw data generated for the EspA protein detection. B) Raw data for DnaK protein that was used as a loading control. The data was generated by normalizing the experiments to the respective $\mathrm{NaCl}$ controls. In the case of LG DMEM (\#1), the control was LB, while in the case of the SCFA conditions, the control was the corresponding $\mathrm{NaCl}$ concentrations. This data is generated from 3 biological replicates. Data are presented as means \pm s.d.; $n=3 .{ }^{*} P \leq 0.05 ;{ }^{* *} P \leq 0.01 ;{ }^{* * *} P \leq$ 0.001. All $P$ values were calculated using Tukey’s Multiple Comparison Test. 


\subsection{GFP Reporter Assay of esp Promoter Activity}

A translational fusion of the esp promoter that is located immediately anterior to the espA gene within the LEE4 genetic region was engineered. The gene region was ligated ahead of GFP, such that whenever the promoter is active, GFP will be transcribed, instead of espA. This construct was cloned in a high copy plasmid pCR2.1-TOPO and transferred into 86-24 $\triangle \operatorname{esp} A$ isogenic mutant such that the bacteria does not produce any form of EspA protein, only the GFP product as activated by the $e s p$ promoter within the LEE4 genetic region. The final construct was confirmed via sequencing data ( Figure $33 \&$ Figure 34 ).

\subsubsection{Activity of esp Promoter Increases at $172 \mathrm{mM}$ SCFA in Low Glucose DMEM High Oxygen}

The first condition tested after the completion of the GFP engineered strains was the low glucose (LG) Dulbecco's Modified Eagle's Medium (DMEM) under the high oxygen condition. A media with low glucose was chosen as the gastrointestinal tract is a low glucose and low nutrient environment. ${ }^{175,176}$ The activity of the esp promoter was monitored during exponential phase and stationary phase. The sample of activity representing exponential phase was taken at 3 hours while the stationary phase snapshot was taken at 9 hours. The entire length of the experiment spanned 17 hours. The data generated is altered from the original raw data in that the control wells that contained media alone were subtracted from all the experimental wells, corrected relative fluorescence units (RFU) were divided by the corrected optical density (OD) reads which generated the graphs in the figures below (Figure 9-Figure 16)

There was a significant increase in esp promoter activity in the exponential phase of the bacteria that was grown with $172 \mathrm{mM}$ short chain fatty acid, compared to that grown in LG DMEM alone and the bacteria grown with $30 \mathrm{mM} \mathrm{SCFA}$ alone (Figure $9-\mathrm{B}$ ). The promoter 
activity under the LG DMEM condition alone was $8.1 \times 10^{4} \mathrm{RFU} / \mathrm{OD}_{600}$. The esp promoter activity with the presence of $30 \mathrm{mM}$ SCFA in LG DMEM was $1.1 \times 10^{5} \mathrm{RFU} / \mathrm{OD}_{600}$. Lastly the esp promoter activity under the $172 \mathrm{mM}$ SCFA treatment was $2.6 \times 10^{5} \mathrm{RFU} / \mathrm{OD}_{600}$ more than double the expression of the $30 \mathrm{mM}$ SCFA condition, and just over 3 times larger than the LG DMEM condition. There was no change in the esp promoter activity between the various treatments in exponential phase (Figure $9-\mathrm{A} \& \mathrm{~B}$ ). It is important to note that the growth rates of the three conditions differed when observing the $\mathrm{OD}_{600}$ growth (Figure $9-\mathrm{C}$ ). During exponential phase, the growth of EHEC in LG DMEM was higher than that in $30 \mathrm{mM}$ SCFA, and the growth of EHEC in $30 \mathrm{mM}$ SCFA was higher than that in $172 \mathrm{mM} \mathrm{SCFA.} \mathrm{In} \mathrm{stationary}$ phase, however, the growth of the bacteria in $172 \mathrm{mM} \mathrm{SCFA}$ was higher than both LG DMEM alone and $30 \mathrm{mM}$ SCFA.

Collectively, this data shows that when EHEC 86-24_espA is exposed to concentrations of SCFA most resembling the large intestine, the activity of the esp promoter is the highest. This promoter activity is significantly higher than that observed under the concentration of SCFA most resembling the small intestine, as well as the low glucose (LG) DMEM media alone. This data is important and novel as it identifies SCFA to be an environmental cue that affects the transcription of T3SS associated genes, particularly EspA. 


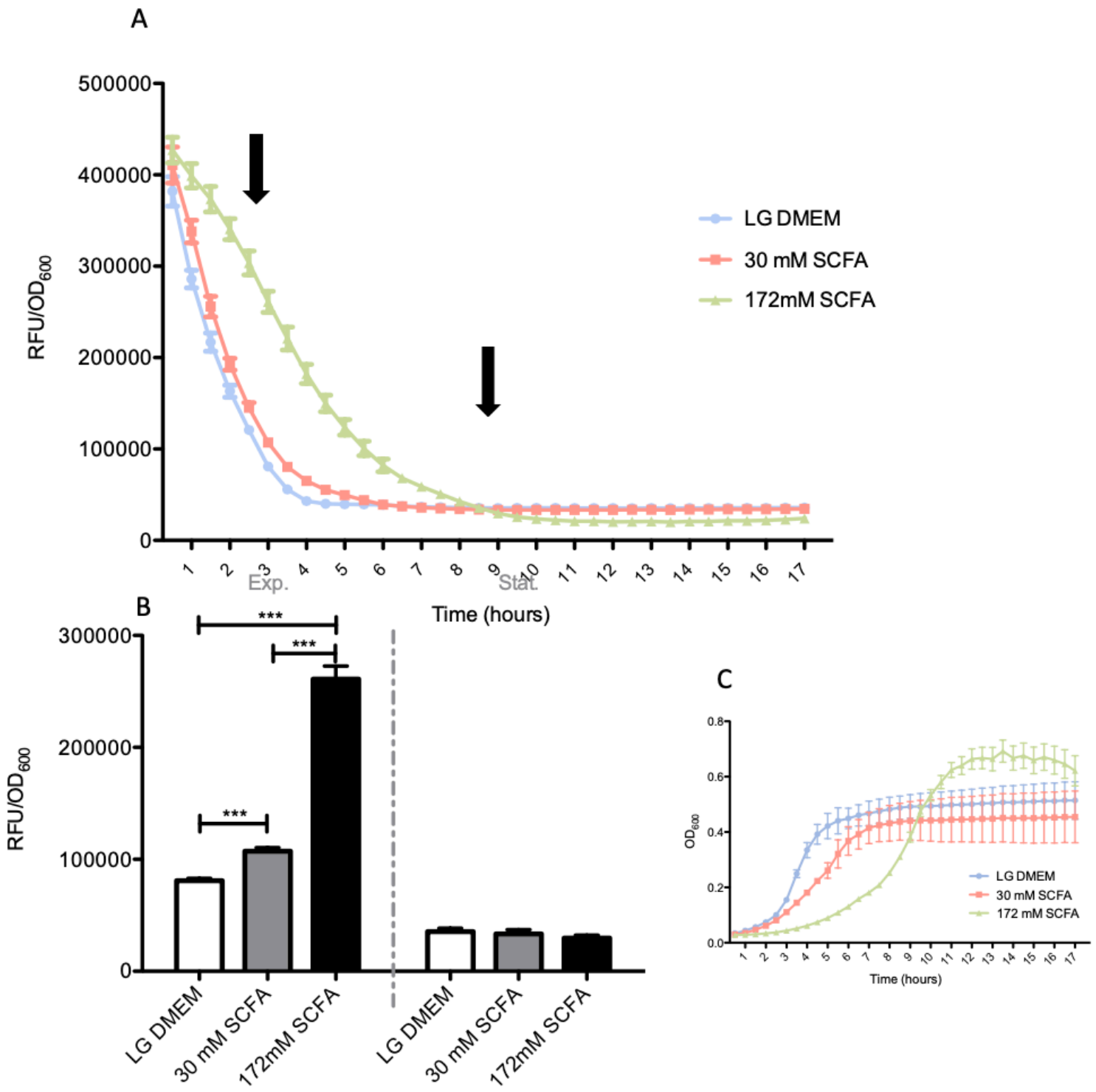

Figure 9. Activity of the esp promoter is greater under large intestinal SCFA condition

EspA expression was monitored using the GFP reporter strain. A) The activity of the esp promoter is is expressed as an average over the $17 \mathrm{hr}$ experiment. B) The average activity of the esp promoter at exponential phase $(3 \mathrm{hr})$, and stationary phase $(9 \mathrm{hr})$. C) The growth of the bacterial strain under the 3 conditions. This was done over the period of 16 hours in low glucose DMEM supplemented with 50 $\mu \mathrm{g} / \mathrm{ml}$ kanamycin to maintain the selection for the plasmid pCR2.1-espA-GFP. This data is generated from 6 biological replicates and 3 technical replicates. Data are presented as means \pm s.d.; $n=6 .{ }^{*} P \leq$ $0.05 ;{ }^{* *} P \leq 0.01 ;{ }^{* * *} P \leq 0.001$. All $P$ values were calculated using Tukey's Multiple Comparison Test. 


\subsubsection{Activity of esp Promoter Increases at 172 mM SCFA in Low Glucose DMEM Low Oxygen}

Expression of the esp promoter was analyzed under the LG DMEM condition with low oxygen. The low oxygen condition was generated through the use of $25 \mu \mathrm{l}$ of MO overlay, this created a film over the well of the 96 well plate. Since $E$. coli is a facultative anaerobe, the culture would use up most of the available oxygen within the media, at which point the bacteria would switch over to either anaerobic or microaerobic metabolism. ${ }^{177}$ The snapshot of activity during exponential phase was taken at 3 hours while the stationary phase snapshot was taken at 9 hours. The entire length of the experiment spanned 17 hours (Figure $10-\mathrm{A}$ ).

The esp expression observed under LG DMEM with MO overlay was $1.1 \times 10^{5}$ RFU/OD 600 . The value for the LG DMEM with MO was significantly higher than the expression of esp under the $30 \mathrm{mM}$ SCFA LG DMEM MO condition where the value was $1.3 \times 10^{5}$ RFU/OD 600 . While both the LG DMEM with MO and LG DMEM with 30 mM SCFA and MO were significantly lower than the esp expression observed at LG DMEM with $172 \mathrm{mM} \mathrm{SCFA}$ and $\mathrm{MO}$ which was $2.95 \times 10^{5} \mathrm{RFU} / \mathrm{OD}_{600}$ (Figure $10-\mathrm{B}$ ). The values during stationary phase did not differ among the various treatment groups. It is important to note that the growth under the mineral oil condition during exponential phase was very comparable among the 3 different treatments (Figure $10-\mathrm{C}$ ). The growth of the bacteria was significantly lower during stationary phase in the bacteria grown with $172 \mathrm{mM}$ SCFA however (Figure $10-\mathrm{C}$ ).

Taken together, the data shows that the highest activity of the esp promoter is under the large intestinal SCFA concentration of $172 \mathrm{mM}$. Furthermore, the addition of mineral oil to reduce oxygen in the low glucose environment appears to have little effect on the activity of the esp promoter. 

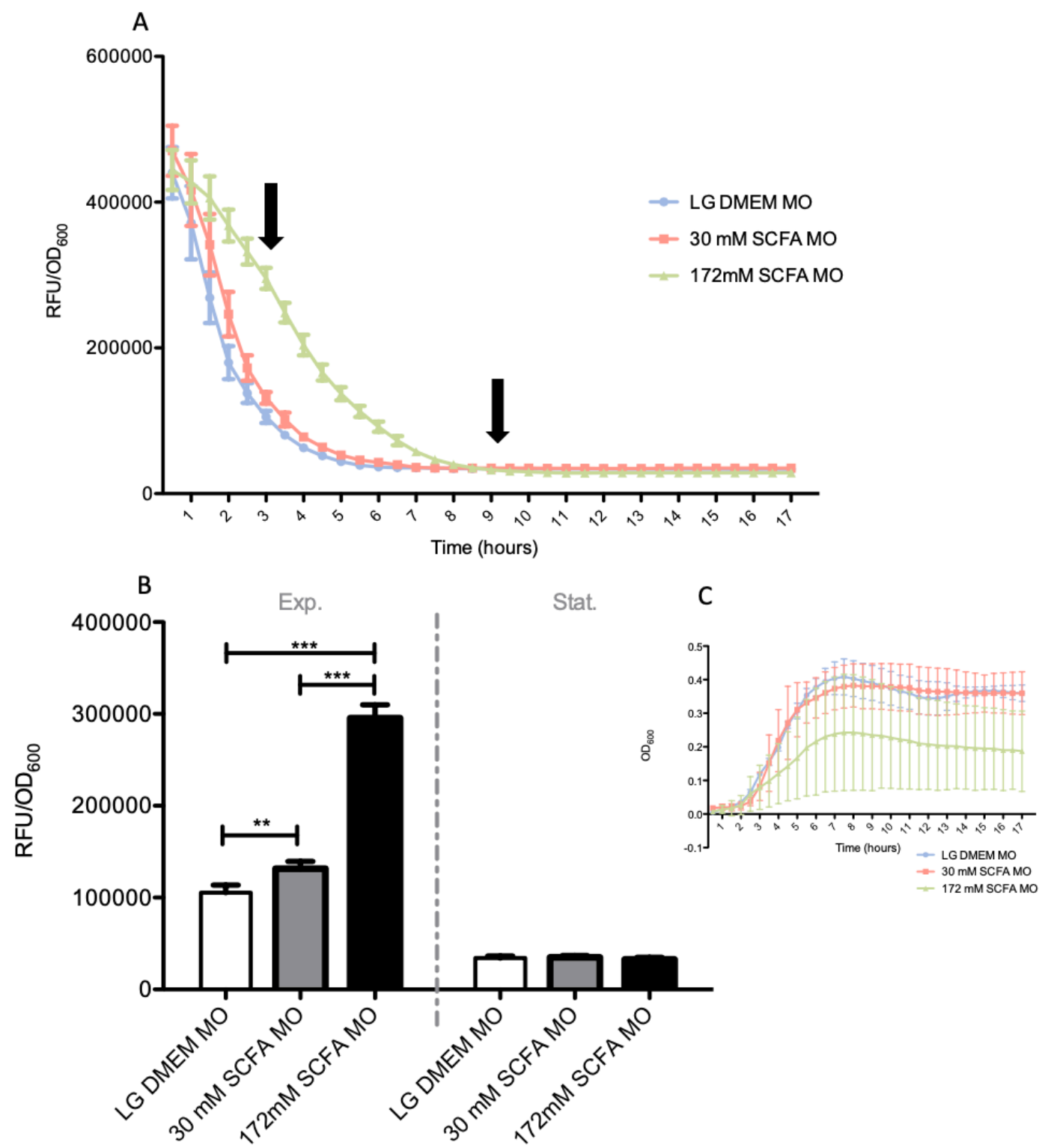

Figure 10. The esp promoter is most active under the large intestinal SCFA condition despite low oxygen

EspA expression is increased in the low oxygen condition when exposed to the large intestinal short chain fatty acid mix, compared to the small intestinal short chain fatty acid mix and no short chain fatty acids at all. A) The activity of the esp promoter is is expressed as an average over the $17 \mathrm{hr}$ experiment. B) The average activity of the esp promoter at exponential phase (3 $\mathrm{hr}$ ), and stationary phase ( $9 \mathrm{hr})$. C) The growth of the bacterial strain under the 3 conditions. This data is generated from 6 biological replicates and 3 technical replicates. Data are presented as means \pm s.d.; $n=6 .{ }^{*} P \leq 0.05 ;{ }^{* *} P \leq 0.01 ;{ }^{* * *} P \leq 0.001$. All $P$ values were calculated using Tukey's Multiple Comparison Test. 


\subsubsection{Activity of esp Promoter Increases at 172 mM SCFA in High Glucose DMEM High Oxygen}

The esp promoter activity was monitored under the condition of high glucose (HG)

DMEM and high oxygen. This experiment allowed the culture to be exposed to oxygen as the 96 well plate was agitated continuously for the entire experiment, which was 17 hours in length.

Despite the difference in condition where there was a greater concentration of glucose in the media, the trend among the SCFA conditions compared to media alone follows the previous experiments conducted in a low glucose environment with lack of oxygen. During the exponential phase, the activity of the esp promoter in HG DMEM alone was $1.1 \times 10^{5}$ RFU/OD 600 (Figure $11-A \& B$ ). This value was significantly lower than the activity of the same promoter, when grown under the same condition but in the presence of $30 \mathrm{mM}$ SCFA where the value was $1.4 \times 10^{5} \mathrm{RFU} / \mathrm{OD}_{600}$ (Figure $11-\mathrm{A} \& \mathrm{~B}$ ). Lastly, the expression of the esp promoter was the highest when the cells were grown in the presence of $172 \mathrm{mM} \mathrm{SCFA}$, the concentration of SCFA most closely resembling that of the large intestine (Figure 11 - A \& B). It is important to note that the growth of the bacteria in the three different conditions should not affect the outcome of these values as the growth is very similar at the 3 hour time point as well as the 9 hour time point (Figure $11-\mathrm{C}$ ).

The activity of the esp promoter was much lower during the stationary phase, where in HG DMEM alone the activity was $3.5 \times 10^{4} \mathrm{RFU} / \mathrm{OD}_{600}$, while in HG DMEM with $30 \mathrm{mM}$ SCFA activity was at $3.1 \times 10^{4} \mathrm{RFU} / \mathrm{OD}_{600}$, and lastly in HG DMEM with $172 \mathrm{mM}$ SCFA the activity was at $2.8 \times 10^{4} \mathrm{RFU} / \mathrm{OD}_{600}$ (Figure $11-\mathrm{B}$ ). No significant difference was observed between the three different conditions. That being said, the expression in all 3 conditions during stationary phase lower than are significantly lower than the expression of the esp promoter during exponential phase. 
It is evident by the data presented that $172 \mathrm{mM} \mathrm{SCFA}$ has an activating effect on the esp promoter. It is also evident that glucose concentration does not play a decisive role in the activity of the esp promoter, or perhaps assembly of the T3SS machinery. 

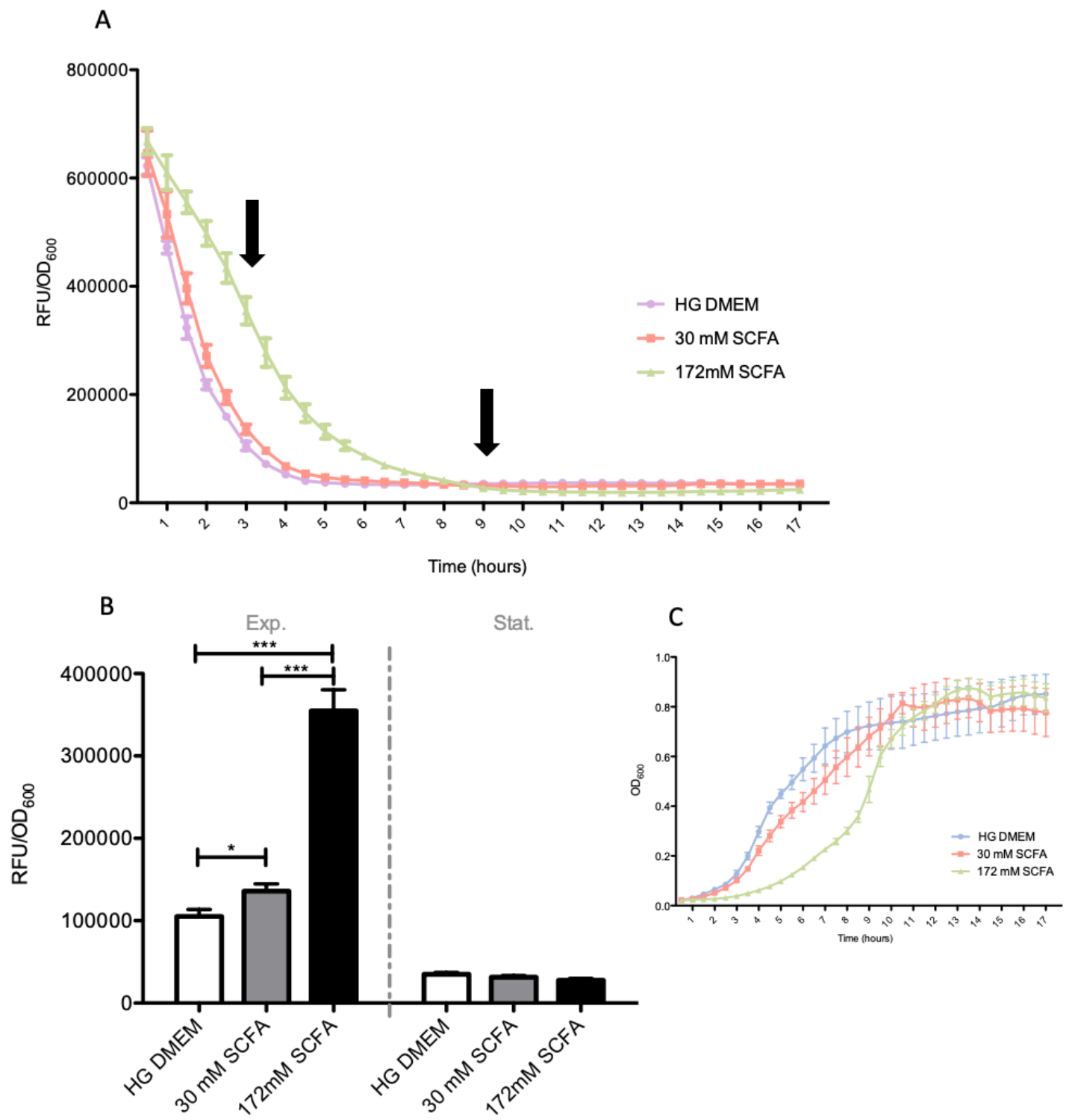

Figure 11. Activity of esp promoter is greatest under the large intestinal condition with high glucose

The graph above is a quantification of EspA expression by GFP abundance of EHEC 86-24 $\triangle \operatorname{esp} A$ in high glucose DMEM and various SCFA. A) The activity of the esp promoter is is expressed as an average over the $17 \mathrm{hr}$ experiment. B) The average activity of the esp promoter at exponential phase $(3 \mathrm{hr})$, and stationary phase $(9 \mathrm{hr})$. C) The growth of the bacterial strain under the 3 conditions. This data is generated from 6 biological replicates and 3 technical replicates. Data are presented as means \pm s.d.; $n=6 .{ }^{*} P \leq 0.05 ;{ }^{* *} P \leq 0.01 ;{ }^{* * *} P \leq 0.001$. All $P$ values were calculated using Tukey's Multiple Comparison Test. 


\subsubsection{Activity of esp Promoter Increases at $172 \mathrm{mM}$ SCFA in High Glucose DMEM Low Oxygen}

The esp promoter was further tested under the high glucose condition in the absence of oxygen. In the exponential phase, under the HG DMEM condition alone the activity of esp was $1.1 \times 10^{5} \mathrm{RFU} / \mathrm{OD}_{600}$. The activity in HG DMEM alone was not significantly different from HG DMEM under $30 \mathrm{mM}$ SCFA, yet significantly different from the $172 \mathrm{mM} \mathrm{SCFA}$ treatment. The activity of esp was $1.2 \times 10^{5} \mathrm{RFU} / \mathrm{OD}_{600}$ which was significantly lower than the activity in 172 mM SCFA in HG DMEM. The activity of esp in $172 \mathrm{mM} \mathrm{SCFA}$ in HG DMEM was $3.3 \times 10^{5}$ RFU/OD 600 , nearly three times greater than both HG DMEM and 30 mM SCFA HG DMEM (Figure $12-\mathrm{A} \& \mathrm{~B}$ ).

In stationary phase, the activity of the esp promoter was significantly lower than that of all the conditions in exponential phase. In HG DMEM alone the expression was $3.7 \times 10^{4}$ RFU/OD 600 . In the HG DMEM with $30 \mathrm{mM}$ SCFA condition, the expression of esp was $3.4 \mathrm{x}$ $10^{4} \mathrm{RFU} / \mathrm{OD}_{600}$. Lastly, in HG DMEM with $172 \mathrm{mM}$ SCFA the expression of esp was $3.2 \times 10^{4}$ RFU/OD 600 . Within the stationary phase, there was no significant difference among the three different conditions (Figure $12-\mathrm{A} \& \mathrm{~B}$ ).

The growth of the bacteria under the three various treatment groups slightly varied particularly during the exponential phase. The growth in the $172 \mathrm{mM} \mathrm{SCFA} \mathrm{treatment} \mathrm{group}$ grew slower than the HG DMEM and HG DMEM 30 mM SCFA treatments. The growth was more similar during the stationary phase.

Based on the data presented, large intestinal concentrations of SCFA have a stimulating effect on the esp promoter. This effect is irrespective of glucose concentration, nor oxygen concentration in the case of the data presented. 

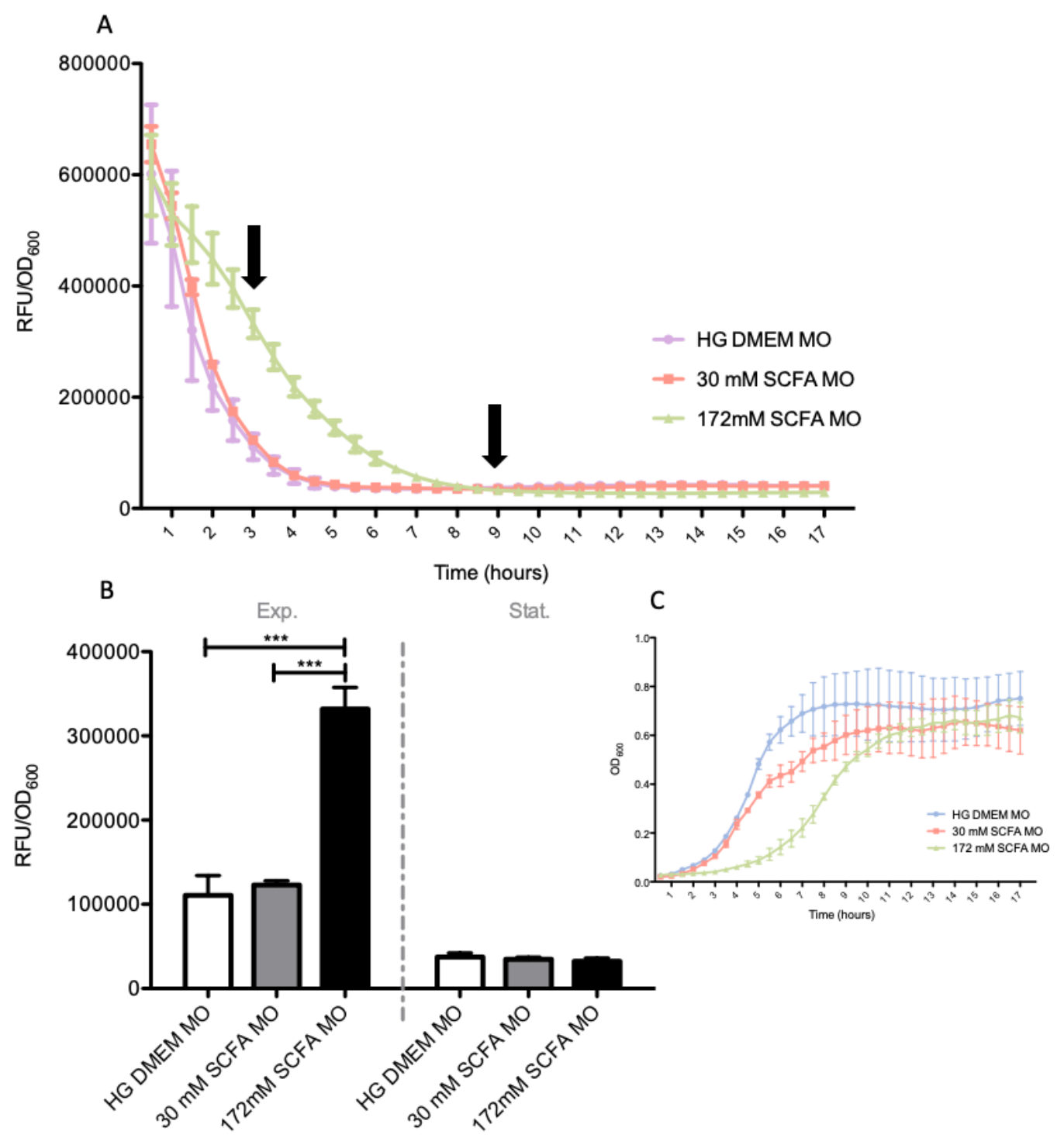

Figure 12. Activity of esp promoter in high glucose DMEM, low oxygen and various SCFA

Expression of EspA is unchanged under various short chain fatty acid conditions in the high glucose and low oxygen environment. A) The activity of the esp promoter is is expressed as an average over the $17 \mathrm{hr}$ experiment. B) The average activity of the esp promoter at exponential phase $(3 \mathrm{hr})$, and stationary phase $(9 \mathrm{hr})$. C) The growth of the bacterial strain under the 3 conditions. This data is generated from 6 biological replicates and 3 technical replicates. Data are presented as means \pm s.d.; $n=6 .{ }^{*} P \leq 0.05 ;{ }^{* *} P \leq 0.01 ;{ }^{* * *} P \leq 0.001$. All $P$ values were calculated using Tukey's Multiple Comparison Test. 


\subsubsection{Activity of esp Promoter Increases at 172 mM SCFA in M9 Media with Low Magnesium}

The activity of the esp promoter is generally lower in EHEC O157:H7 cells grown in M9 media with low magnesium when compared to either LG or HG DMEM. During exponential phase the activity in M9 Low $\mathrm{Mg}^{2+}$ is $3.7 \times 10^{3} \mathrm{RFU} / \mathrm{OD}_{600}$. The activity of esp during exponential phase in bacteria grown in M9 Low $\mathrm{Mg}^{2+}$ with $30 \mathrm{mM}$ SCFA is $3.5 \times 10^{3}$ RFU/OD 600 , which is similar to that of M9 Low $\mathrm{Mg}^{2+}$ alone. Bacteria grown in M9 Low $\mathrm{Mg}^{2+}$ with $172 \mathrm{mM}$ SCFA possess esp activity significantly higher than both M9 Low $\mathrm{Mg}^{2+}$ alone and M9 Low $\mathrm{Mg}^{2+}$ with $30 \mathrm{mM}$ SCFA. The value for bacteria grown in M9 Low $\mathrm{Mg}^{2+}$ with $172 \mathrm{mM}$ SCFA is $6.3 \times 10^{3} \mathrm{RFU} / \mathrm{OD}_{600}$ (Figure $13-\mathrm{A} \& \mathrm{~B}$ ). Yet again the $172 \mathrm{mM} \mathrm{SCFA}$ treatment displayed the highest expression within the EHEC O157:H7 strain 86-24 bacterial cells.

The stationary phase activity was reversed from the trend in the exponential phase, where a higher activity was observed in the M9 Low $\mathrm{Mg}^{2+}$ alone and M9 Low $\mathrm{Mg}^{2+}$ with $30 \mathrm{mM}$ SCFA, with significantly lower activity in the M9 Low $\mathrm{Mg}^{2+}$ with $172 \mathrm{mM}$ SCFA. The activity in M9 Low $\mathrm{Mg}^{2+}$ alone during stationary phase was $9.6 \times 10^{3} \mathrm{RFU} / \mathrm{OD}_{600}$, significantly higher than M9 Low $\mathrm{Mg}^{2+}$ in exponential phase and higher than M9 Low $\mathrm{Mg}^{2+}$ with $172 \mathrm{mM}$ SCFA during stationary phase. The activity in M9 Low $\mathrm{Mg}^{2+}$ with $30 \mathrm{mM}$ SCFA was $1.1 \times 10^{4}$ RFU/OD 600 , which was significantly higher than M9 Low $\mathrm{Mg}^{2+}$ with $30 \mathrm{mM}$ SCFA in exponential phase, and higher than $4.7 \times 10^{3} \mathrm{RFU} / \mathrm{OD}_{600}$, the value of expression in M9 Low $\mathrm{Mg}^{2+}$ with $172 \mathrm{mM}$ SCFA during stationary phase (Figure 13 - A \& B).

It is important to note the variability in growth rates among the various treatments (Figure $13-\mathrm{C})$. The condition that involves M9 Low $\mathrm{Mg}^{2+}$ with $172 \mathrm{mM}$ SCFA had slightly slower growth compared to the M9 Low $\mathrm{Mg}^{2+}$ alone, and M9 Low $\mathrm{Mg}^{2+}$ with $30 \mathrm{mM}$ SCFA. 
Conversely, the growth was greater in M9 Low $\mathrm{Mg}^{2+}$ with $172 \mathrm{mM}$ SCFA condition during exponential phase (Figure $13-\mathrm{C}$ ).

Altogether, it is evident that $172 \mathrm{mM} \mathrm{SCFA} \mathrm{concentration} \mathrm{likely} \mathrm{found} \mathrm{in} \mathrm{the} \mathrm{large}$ intestine has an activating effect on the esp promoter in the low $\mathrm{Mg}^{2+}$ condition during exponential phase. Interestingly, this effect is reversed during stationary phase, where activity of the esp promoter is lowest under the $172 \mathrm{mM}$ SCFA concentration. 

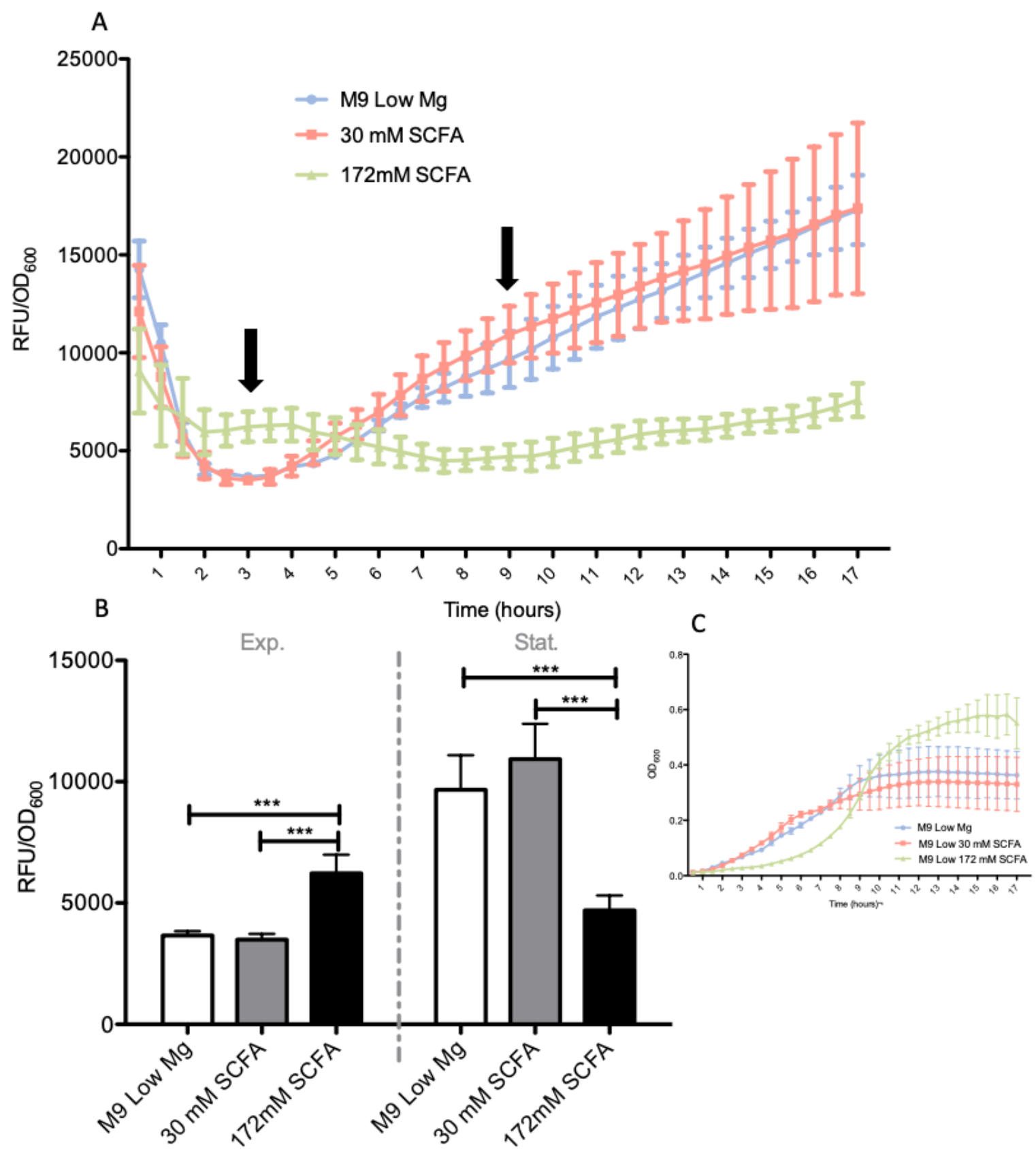

Figure 13. esp promoter activity under the influence of low magnesium and SCFA

Expression of EspA increases with increasing concentration of SCFA that mimic the large intestine in the low magnesium environment. A) The activity of the esp promoter is is expressed as an average over the $17 \mathrm{hr}$ experiment. B) The average activity of the esp promoter at exponential phase ( $3 \mathrm{hr})$, and stationary phase $(9 \mathrm{hr})$. C) The growth of the bacterial strain under the 3 conditions. This data is generated from 6 biological replicates and 3 technical replicates. Data are presented as means \pm s.d.; $n=6 .{ }^{*} P \leq 0.05 ;{ }^{* *} P \leq 0.01 ;{ }^{* * *} P \leq$ 0.001. All $P$ values were calculated using Tukey’s Multiple Comparison Test. 


\subsubsection{Activity of esp Promoter Increases at $172 \mathrm{mM}$ SCFA in M9 Media with High Magnesium}

The activity of the esp promoter that precedes the espA gene region within the LEE4 operon was assessed in M9 media containing high magnesium. The overall expression was lower than the expression of this promoter under both low glucose and high glucose DMEM (Figure 14 $-B)$.

During exponential phase, the data indicated very low expression within all the treatment groups. In the M9 $\mathrm{High} \mathrm{Mg}^{2+}$ alone, the expression of the esp promoter was $2.1 \times 10^{3}$ RFU/OD 600 which was significantly lower than activity in M9 High $\mathrm{Mg}^{2+}$ with $172 \mathrm{mM}$ SCFA. The activity in M9 High $\mathrm{Mg}^{2+}$ with $30 \mathrm{mM}$ SCFA was $2.3 \times 10^{3} \mathrm{RFU} / \mathrm{OD}_{600}$ which was also significantly lower than that of M9 High $\mathrm{Mg}^{2+}$ with $172 \mathrm{mM}$ SCFA condition. The M9 High $\mathrm{Mg}^{2+}$ with $172 \mathrm{mM}$ SCFA condition harbored an activity of $4.1 \times 10^{3} \mathrm{RFU} / \mathrm{OD}_{600}$, nearly twice that of both M9 High $\mathrm{Mg}^{2+}$ alone and M9 High $\mathrm{Mg}^{2+}$ with 30 mM SCFA (Figure $14-\mathrm{A} \& \mathrm{~B}$ ).

During stationary phase, the trend was reversed as in the low magnesium condition. The activity of the esp promoter under M9 High $\mathrm{Mg}^{2+}$ alone and M9 $\mathrm{High}_{\mathrm{Mg}^{2+}}$ with $30 \mathrm{mM}$ SCFA was significantly higher than that of the M9 $\mathrm{High} \mathrm{Mg}^{2+}$ with $172 \mathrm{mM} \mathrm{SCFA}$ (Figure 14 - A \& B). The exact value for the esp promoter activity in $\mathrm{M} 9 \mathrm{High} \mathrm{Mg}^{2+}$ alone during stationary phase was $1.4 \times 10^{4} \mathrm{RFU} / \mathrm{OD}_{600}$. The value during stationary phase in the $\mathrm{M} 9 \mathrm{High} \mathrm{Mg}^{2+}$ with $30 \mathrm{mM}$ SCFA condition was also $1.4 \times 10^{4} \mathrm{RFU} / \mathrm{OD}_{600}$. While the value for the activity of esp in the M9 High $\mathrm{Mg}^{2+}$ with $172 \mathrm{mM}$ SCFA condition was almost half at $7.3 \times 10^{3} \mathrm{RFU}^{-\mathrm{OD}_{600}}$ (Figure $14-\mathrm{A}$ $\& \mathrm{~B})$.

The growth rates of the strain 86-24 under the various conditions were accounted and slightly varied among the 3 conditions (Figure $14-\mathrm{C}$ ). The growth was slightly stunted by the 
presence of the $172 \mathrm{mM}$ SCFA during exponential phase. In stationary phase the growth for the $172 \mathrm{mM}$ SCFA condition was higher than M9 High $\mathrm{Mg}^{2+}$ and M9 High $\mathrm{Mg}^{2+}$ with $30 \mathrm{mM}$ SCFA.

All things considered, the effect that $172 \mathrm{mM}$ SCFA has on the activity of the esp promoter is significantly greater than that exerted by $30 \mathrm{mM}$ SCFA concentration or M9 high $\mathrm{Mg}^{2+}$ media alone. The same effect as in the low $\mathrm{Mg}^{2+}$ media is seen during stationary phase, where the activity of the promoter is diminished in the $172 \mathrm{mM} \mathrm{SCFA}$ and increased in M9 media alone and $30 \mathrm{mM}$ SCFA. The fact that the expression profile in low and high $\mathrm{Mg}^{2+}$ is constant, is indicative of the fact that PhoP-PhoQ two component regulatory system is not involved in the regulation of the T3SS gene, EspA. 

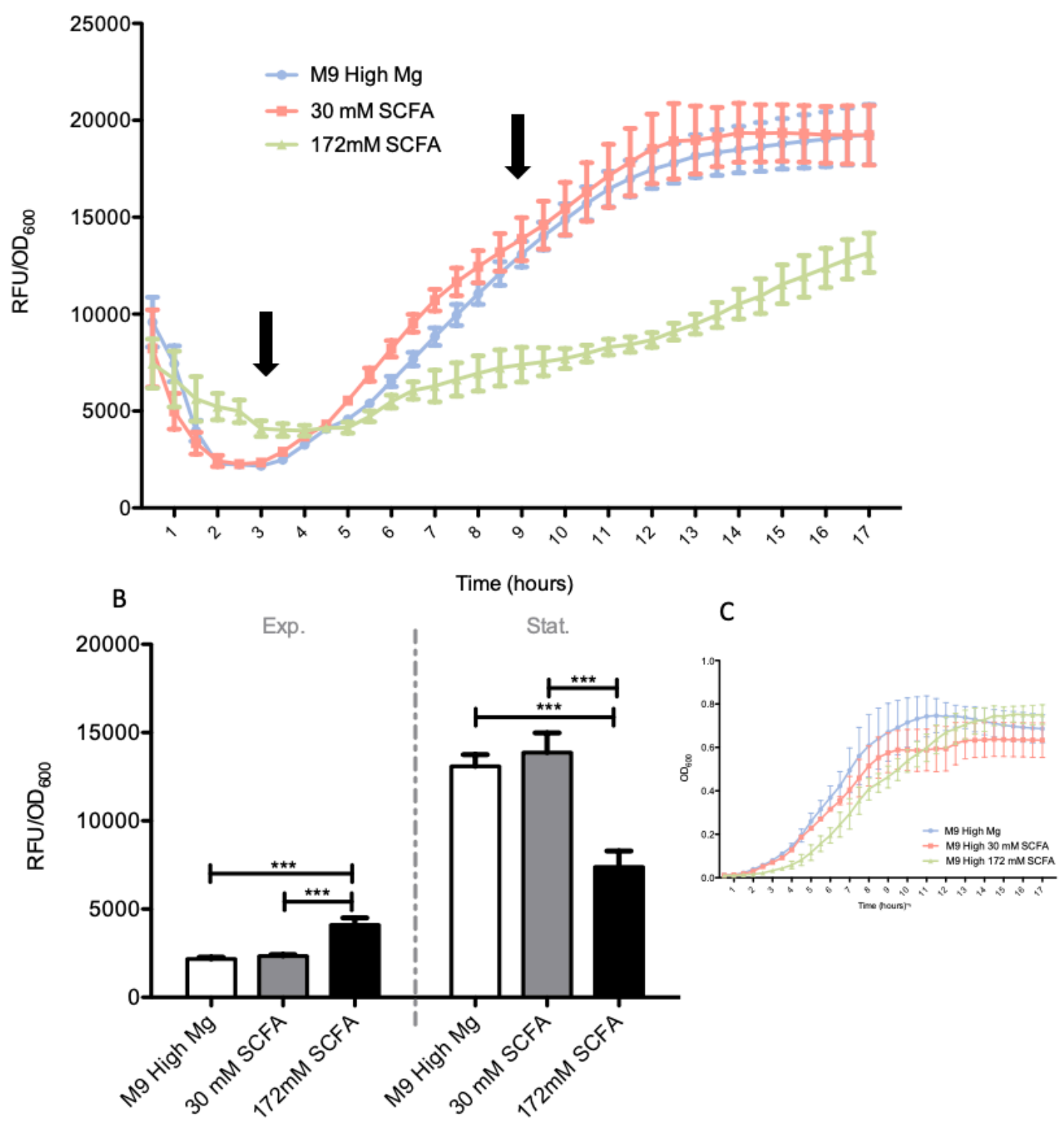

Figure 14. esp promoter activity under the influence of high magnesium and SCFA

Expression of EspA increases with increasing concentration of SCFA that mimic the large intestine in the high magnesium environment. A) The activity of the esp promoter is is expressed as an average over the $17 \mathrm{hr}$ experiment. B) The average activity of the esp promoter at exponential phase $(3 \mathrm{hr})$, and stationary phase $(9 \mathrm{hr})$. C) The growth of the bacterial strain under the 3 conditions. This data is generated from 6 biological replicates and 3 technical replicates. Data are presented as means \pm s.d.; $n=6 .{ }^{*} P \leq 0.05 ;{ }^{* *} P \leq 0.01 ;{ }^{* * *} P \leq 0.001$. All $P$ values were calculated using Tukey's Multiple Comparison Test. 


\subsubsection{Activity of esp Promoter Increases due to Higher Glucose in $\mathrm{H}_{2} \mathrm{O}_{2}$}

Reactive oxygen species (ROS) are important players during an inflammation, for this reason the effect of hydrogen peroxide $\left(\mathrm{H}_{2} \mathrm{O}_{2}\right)$ was tested on EHEC O157:H7 strain 86-24_espA containing GFP labelled esp promoter. Since this promoter is associated with virulence gene $\operatorname{esp} A$, this is valuable data to understand how this virulence gene would react under exposure to (ROS).

Exponential phase expression of esp was highest in HG DMEM, while being lowest in LB (Figure $15-\mathrm{A} \& \mathrm{~B}$ ). The exact value for esp promoter activity in LB was $1.1 \times 10^{5}$ $\mathrm{RFU} / \mathrm{OD}_{600}$, which was significantly lower than that of LG DMEM as well as HG DMEM. The value of $e s p$ promoter activity in LG DMEM was $3.3 \times 10^{5} \mathrm{RFU} / \mathrm{OD}_{600}$. The HG DMEM condition harbored an expression of $4.6 \times 10^{5} \mathrm{RFU} / \mathrm{OD}_{600}$, expression over 4 times greater than that of the LB condition (Figure 15 - A \& B). Generally speaking, the activity of the promoter was significantly higher when exposed to ROS than when the bacteria was grown in M9 minimal media.

Stationary phase expression was much lower. There was no significant difference among the three treatment conditions (Figure 15 - A \& B). The value for stationary phase expression in LB was $1.9 \times 10^{4} \mathrm{RFU} / \mathrm{OD}_{600}$. For the LG DMEM condition the value was $9.2 \times 10^{4} \mathrm{RFU} / \mathrm{OD}_{600}$. While for the HG DMEM condition the value was $8.5 \times 10^{4} \mathrm{RFU} / \mathrm{OD}_{600}$.

The growth of the bacteria under these various conditions differed, particularly for the LG DMEM condition (Figure $15-C$ ). The bacteria grew the best in LB, with a decreased growth observed in the HG DMEM and an even further decrease in the LG DMEM (Figure 15 C). 
All in all, the highest activity of the esp promoter was seen in the high glucose condition in combination with ROS during exponential phase. 

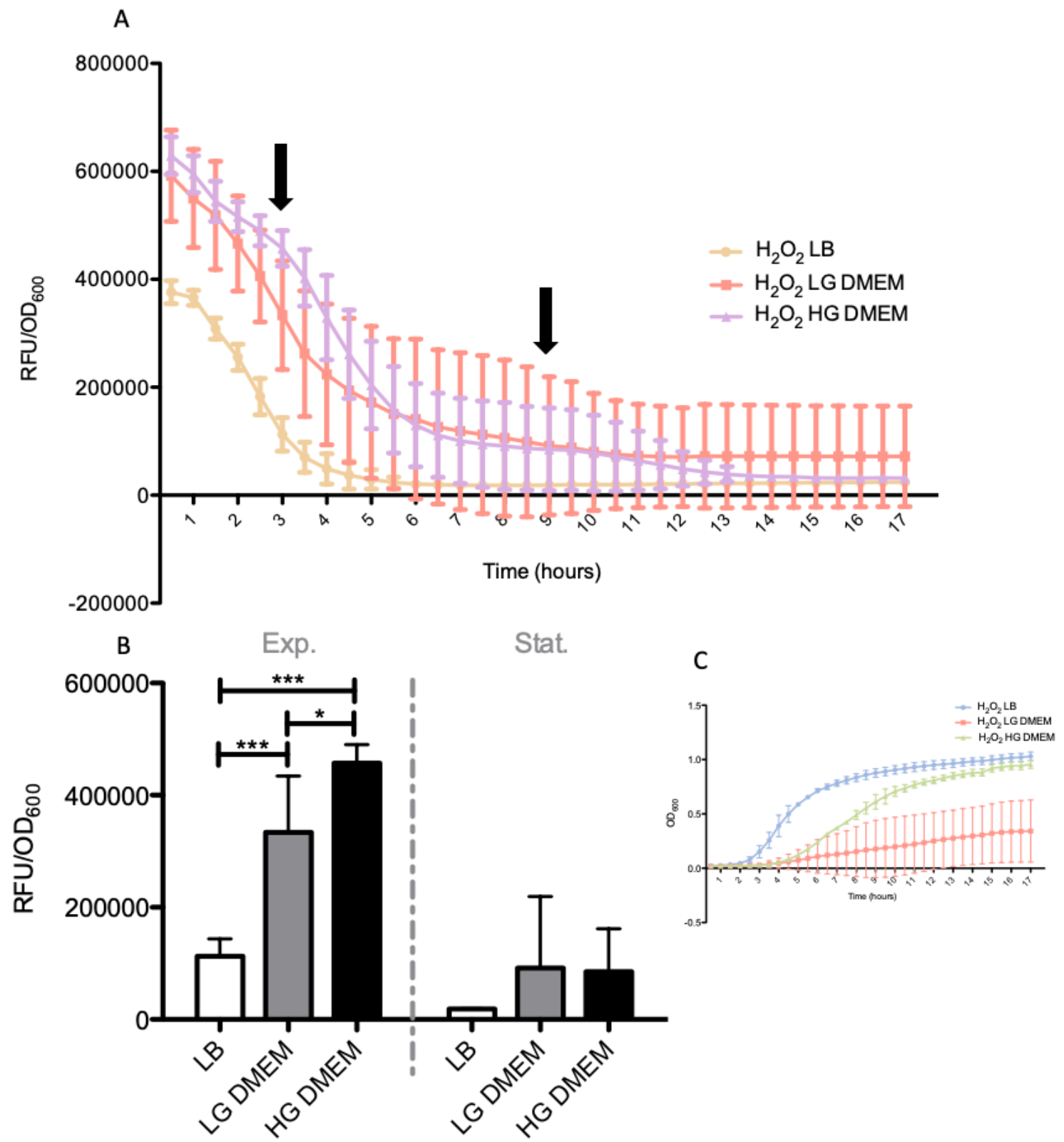

Figure 15. Effect of reactive oxygen species on esp promoter activity

Expression of EspA is down regulated in LB, low glucose DMEM and high glucose DMEM when exposed to $0.2 \%$ hydrogen peroxide. A) The activity of the esp promoter is is expressed as an average over the $17 \mathrm{hr}$ experiment. B) The average activity of the esp promoter at exponential phase ( $3 \mathrm{hr})$, and stationary phase $(9 \mathrm{hr})$. C) The growth of the bacterial strain under the 3 conditions. This data is generated from 6 biological replicates and 3 technical replicates. Data are presented as means \pm s.d.; $n=6 .{ }^{*} P \leq 0.05$; $^{* *} P \leq 0.01$; ${ }^{* * *} P \leq 0.001$. All $P$ values were calculated using Tukey's Multiple Comparison Test. 


\subsubsection{Activity of esp Promoter Increases in Low Biotin and Restricted $\mathrm{O}_{2}$ Environment}

The effect of biotin was tested on the expression of the esp promoter. Biotin is known to have inhibitory effects on the LEE encoded genes in high concentrations $(>100 \mathrm{nM})^{178}$ that mimic those found in the small intestine. For this reason, the concentration of $30 \mathrm{nM}$ biotin was tested as research suggests this concentration is close to what would be bioavailable to the bacteria in the large intestine.

During the exponential phase of growth, exposure to $30 \mathrm{nM}$ of biotin in LG DMEM generated an expression of $1.0 \times 10^{4} \mathrm{RFU} / \mathrm{OD}_{600}$. This value was significantly lower than that observed under low oxygen conditions (Figure 16-A \& B). The value observed when the bacteria were incubated with $30 \mathrm{nM}$ biotin and MO overlay was $4.9 \times 10^{4} \mathrm{RFU} / \mathrm{OD}_{600}$. This value is almost 5-fold greater than that of the experiment under full oxygen condition.

The stationary phase of growth showed a similar trend, where promoter activity was significantly greater in both biotin conditions compared to their corresponding LG DMEM alone. The value of esp promoter activity when grown in LG DMEM and exposed to $30 \mathrm{nM}$ of biotin was $1.0 \times 10^{4} \mathrm{RFU} / \mathrm{OD}_{600}$. This value was significantly lower than that of the esp promoter in LG DMEM, exposed to $30 \mathrm{nM}$ of biotin in low oxygen, which harbored a value of $1.5 \times 10^{4}$ RFU/OD 600 (Figure $16-\mathrm{A} \& \mathrm{~B})$.

The growth of EHEC O157:H7 strain 86-24 $\operatorname{esp} A+$ pCR2.1-espA-GFP was monitored over the course of the 17-hour experiment (Figure 16-C). There was no observable difference in the growth of the bacteria neither at exponential phase nor at stationary phase. The bacteria had very similar growth trends under either condition.

Collectively, this data shows that low biotin concentrations are a powerful environmental cue, particularly in combination with restrictive oxygen concentrations. 

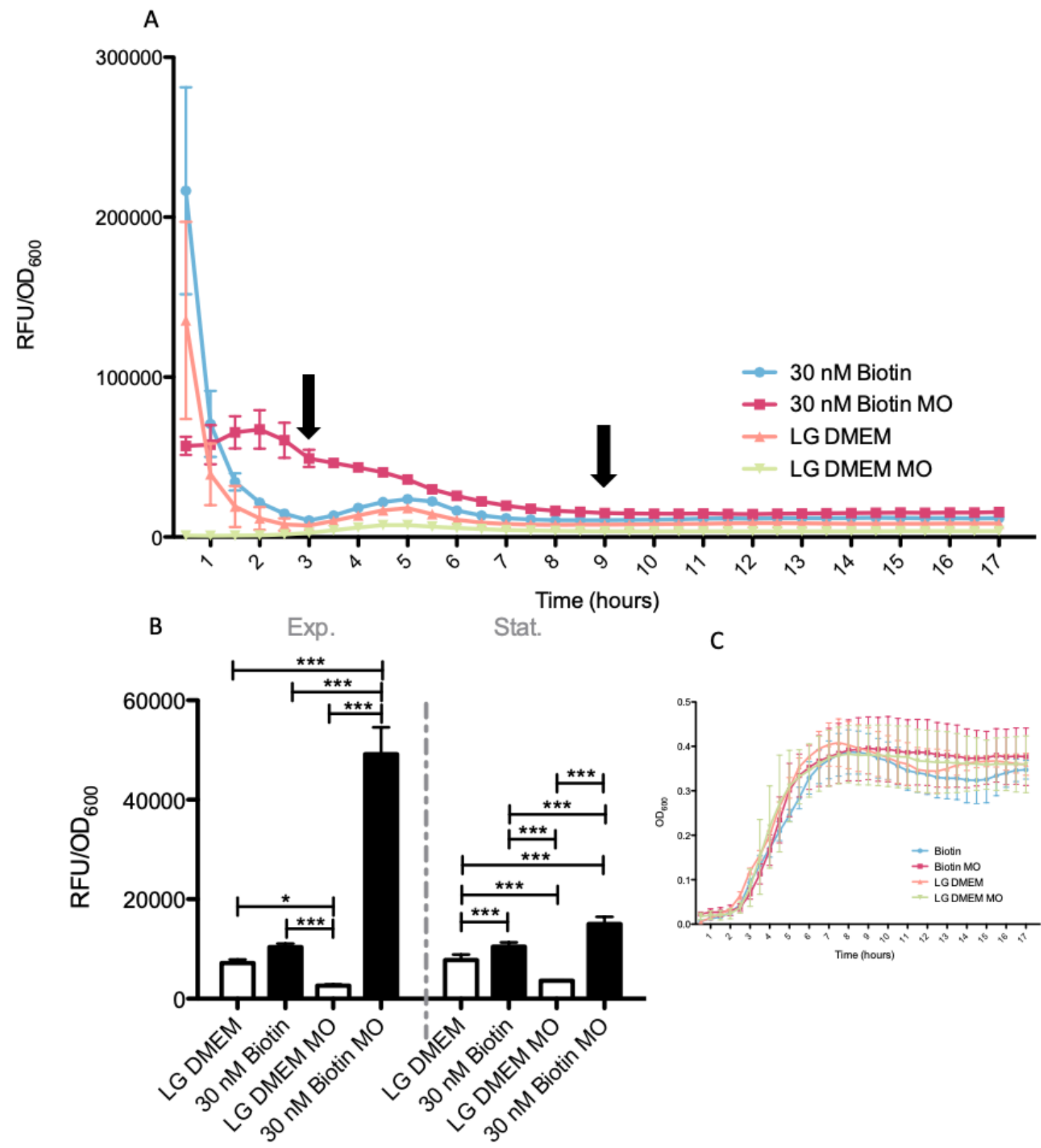

\section{Figure 16. Effect of biotin on esp promoter activity}

The data shows a significant increase in esp promoter activity under the low oxygen condition in combination with biotin. This trend continues into the stationary phase A) The activity of the esp promoter is is expressed as an average over the $17 \mathrm{hr}$ experiment. B) The average activity of the esp promoter at exponential phase $(3 \mathrm{hr})$, and stationary phase $(9 \mathrm{hr})$. C) The growth of the bacterial strain under the 3 conditions. This data is generated from 6 biological replicates and 3 technical replicates. Data are presented as means \pm s.d.; $n=6 .{ }^{*} P \leq 0.05 ;{ }^{* *} P \leq 0.01 ;{ }^{* * *} P$ $\leq 0.001$. All $P$ values were calculated using Tukey's Multiple Comparison Test. 
The data generated in Results Section 3.5 elegantly demonstrated the importance of short chain fatty acid (SCFA) concentration, biotin concentration and oxygen concentration on the activity of the esp promoter. The activity of the esp promoter is greatest when EHEC O157:H7 is exposed to large intestinal concentrations of SCFA (172 mM) (Figure 17). Furthermore, under low biotin levels typical of the large intestine, the promoter activity of esp increased. This increase is most prominent under the low oxygen condition in low glucose (LG) DMEM with 30 $\mathrm{nM}$ biotin, conditions which are typical of the large intestine (Figure 17).

\begin{tabular}{|c|c|c|c|c|c|c|}
\hline \multirow{2}{*}{ Treatment } & Low Glucose & Low Glucose & High Glucose & High Glucose & M9 Media & M9 Media \\
\hline & $\mathrm{High} \mathrm{O}_{2}$ & Low $\mathrm{O}_{2}$ & $\mathrm{High} \mathrm{O}_{2}$ & Low $\mathrm{O}_{2}$ & $\mathrm{High} \mathrm{Mg}^{2+}$ & Low $\mathrm{Mg}^{2+}$ \\
\hline No stress & 80919.1 & 105312.6 & 105261.1 & 110743.2 & 2170.3 & 3666.6 \\
\hline 30 mM SCFA & 107171.3 & 131433.8 & 135855.5 & 123018.5 & 2340.3 & 3489.0 \\
\hline 172 mM SCFA & 261010.9 & 295401.5 & 354871.3 & 331999.5 & 4099.3 & 6224.5 \\
\hline
\end{tabular}

\section{High}

Low Activity

Activity

Figure 17. Heat map of GFP reporter data of esp promoter activity in EHEC 0157:H7

The values represented within the heat map are averages of 3 technical replicates, and 6 biological replicates for each of the conditions tested for the strains tested in the no stress, ileal stress, and colonic stress. The values are averages expressed in units of RFU/OD 600 . The reactive oxygen species (ROS) is separate from the rest of the conditions tested as it was tested under 3 various media rather than 3 concentrations of SCFA. The biotin data was not compared with SCFA treatment therefore could not be incorporated in this figure. 


\subsection{DISCUSSION}

EHEC has evolved a complex regulatory network of genetic expression, particularly when it comes to LEE regulation. Several levels of hierarchical signal transduction pathways are involved in altering genetic expression in EHEC. These hierarchical pathways are further convoluted by their involvement with intracellular regulatory proteins. Motility is not a virulence factor independently, yet the regulation of motility appears to play an essential role in preserving energy in order for EHEC to be capable of successful infection once this pathogen has made its way to the site of colonization. ${ }^{130}$ The hypothesis being that once EHEC reached the colon, the pathogen up-regulates virulence genes such as phage encoded Stx, and T3SS genes located in the LEE PAI. An additional benefit to control of flagellum is that flagellation permits the pathogen to swim in close juxtaposition to the colonic epithelium. Once close to the colonic epithelium, EHEC can potentially use environmental cues to express LEE PAI T3SS genes which allow for the formation of intimate attachment to colonic epithelium via A/E lesion formation. ${ }^{179,180}$ Once successful attachment, and $\mathrm{A} / \mathrm{E}$ lesion is formed, eventually Stx production is triggered within the pathogen where Stx is released through the process of bacterial autolysis. ${ }^{181,182}$ This infection process leads to host morbidity and mortality through the establishment of hemorrhagic colitis and HUS.

The coordination of this infection cycle is orchestrated through EHEC's ability to sense both biotic cues the likes of host epinephrine, norepinephrine, catecholamines, cationic antimicrobial peptides (CAMPs), as well as abiotic cues, such as oxygen, biotin, magnesium, short chain fatty acids (SCFA) to name a few, through the use of two-component sensor kinases. ${ }^{183,184}$ EHEC is also capable of sensing signaling molecules produced by other bacterial 
cells which are referred to as autoinducers (AI). These AI molecules allow EHEC to sense the bacterial population density and diversity in the surrounding environment. ${ }^{184,185}$ EHEC correspondingly regulates it's genetic arsenal by virtue of the recognition and assimilation of aforementioned and many uncharted regulatory pathways and networks.

The objective of this research was to enhance the knowledge of virulence regulation in EHEC by investigating the nature of the T3SS translocon subunit protein EspA (encoded by the esp $A$ gene), and bacterial LEE encoded regulator Ler (encoded by the ler gene) under the influence of various abiotic stimuli, specifically short chain fatty acids (SCFAs). This project is important for several notable reasons: (1) Motility proteins such as FliC have been previously shown to be affected by changing SCFA concentrations from the small intestine to the large intestine $^{130}$ (2) EspA expression has been previously shown to be affected by abiotic cues such as oxygen and biotin in EHEC ${ }^{150,186}$, (3) T3SS are under very complex regulation from many converging regulatory networks. Thus, understating the regulatory pathways is important in understanding of EHEC virulence.

The results generated in this study reveal that the diverse microenvironments along the longitudinal axis of the gut may influence the gene expression of T3SS genes. The results show that the activity of the esp promoter preceding the espA gene, which encodes the EspA protein, is contingent upon the diverse biogeography of the gut. Further, the data suggests that molecules that may be found in the microenvironment where EHEC typically colonizes, act as activators of T3SS genes that are associated with colonization. It is conceivable that the changes noted in gene expression were triggered by a sensor on the EHEC outer membrane. 


\subsection{EHEC Attachment Assays Reveal Significance of EspA Protein for Attachment}

The intention of this investigation was to assess how the attachment of the EHEC O157:H7 mutant strains would vary when exposed to SCFA treatments representative of the small intestine (30 mM SCFA), and the large intestine (172 mM SCFA). Previous research in EHEC has established some facts regarding the role that espA and ler play in attachment. When it comes to the role of $\operatorname{esp} A$, is well established in literature that this gene is important for

attachment. ${ }^{187,188}$ Although transcriptional analysis suggest that Ler plays a central role at regulating the LEE PAI, there are no experiments specifically investigating ler isogenic EHEC mutants to observe how the lack of ler may affect attachment. The novelty of this project would be looking at the effects of SCFA in the absence of the two virulence genes. ${ }^{179}$

EHEC O157:H7 strain 86-24 wild type elicited greater attachment under the LB and LG DMEM conditions compared to the $30 \mathrm{mM}$ SCFA and $172 \mathrm{mM}$ SCFA in LG DMEM. Research has shown using qRT-PCR that mRNA levels of espA decrease in high glucose (HG) DMEM relative to low glucose (LG) DMEM in WT EHEC. The concentration of glucose in LG DMEM is $1 \mathrm{~g} / \mathrm{L}(5.5 \mathrm{mM})$ compared to $4.5 \mathrm{~g} / \mathrm{L}(25 \mathrm{mM})$ in HG DMEM. LB medium does not contain glucose, therefore, it makes sense to see similar attachment as in LG DMEM. The attachment decreased 83 -fold with the addition of the $30 \mathrm{mM}$ SCFA treatment to $1.2 \%$ compared with the 100\% control. When EHEC O157:H7 was grown in 172 mM SCFA, the attachment decreased 7fold to $14.4 \%$ compared to the WT LB control. This trend makes sense as numerous studies have shown that SCFAs can have a toxic effect on bacteria due to entry of nonionized acids into the bacterial cytoplasm. ${ }^{173,189}$ The common result of SCFA-induced toxicity is growth inhibition owing to pleiotropic errors in the cellular processes. ${ }^{190}$ The calculations of $\%$ attachment took 
into account the growth disparities between the treatments. Another reason for lowered attachment under the SCFA conditions could be due to the fact that the bacteria were exposed to the SCFA prior to the infection of HEp-2 cells, not during. This treatment is dissimilar from the bacterial treatment in the GFP reporter assay, since the esp promoter activity was monitored while the bacterial cells were continuously exposed to the SCFA throughout the experiment. This could be a reason why attachment does not increase under the $172 \mathrm{mM}$ SCFA condition, yet the activity of the esp promoter is higher under the $172 \mathrm{mM}$ SCFA condition.

The EHEC O157:H7 strain 86-24 $\Delta$ esp $A$ suffered a precipitous decline in attachment under all conditions tested. Attachment was $0.35 \%$ when $86-24 \Delta e s p A$ strain was grown in LB, 286-fold lower than the WT LB control. The LG DMEM condition had 77-fold lower attachment than WT LB. Attachment was entirely abolished in the $30 \mathrm{mM}$ SCFA condition. Lastly, attachment was almost 12-fold lower in the $172 \mathrm{mM}$ SCFA condition. This data suggests that the EspA protein is absolutely necessary for EHEC O157:H7 strain 86-24 to successfully attach to HEp-2 epithelial cells. There is some data in the literature defining the role that EspA plays in EHEC O157:H7 attachment. ${ }^{54,169}$ Much more data is available on the role of EspA in EPEC attachment. ${ }^{54,65,68,74,151,156,160,191}$ In EPEC EspA was shown to play an important role in attachment, where by deleting the esp $A$ gene, EPEC failed to attach intimately and cause localized effacement on Caco-2 cells. ${ }^{188}$ Researchers in another study raised polyclonal sera against E. coli $\mathrm{O} 157 \mathrm{EspA}$, where they observed inhibition of $\mathrm{A} / \mathrm{E}$ lesions through fluorescence actin staining (FAS) measurements. ${ }^{187}$ The same polyclonal antibodies, however, did not affect adherence of the E. coli $\mathrm{O} 157$ cells to host epithelium. ${ }^{187}$ Other studies in EHEC observed the behaviours of EspA mutants that were missing additional genes and how that affected the bacterial ability to form biofilms. ${ }^{192}$ Researchers concluded that EspA is not necessary for 
biofilm formation, yet CsgA is. ${ }^{192}$ The research publications that specifically look at the absence of espA, and how this might affect attachment of bacteria to host cells, support the data provided in this manuscript. Another publication generated data that showed abolished attachment during infection of HeLa cells when removing the espA gene from EHEC O157:H7 strain EDL933. ${ }^{167}$ Our data supports this finding by demonstrating that when the entirety of the EspA protein coding region is removed from the bacteria, attachment was virtually eliminated.

Remarkably, EHEC O157:H7 strain 86-24Aler attachment was affected and in some

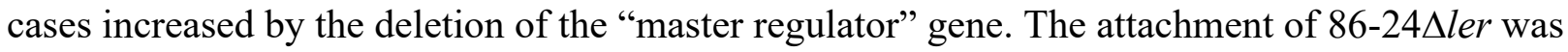
significantly elevated when grown in $172 \mathrm{mM}$ SCFA relative to the $30 \mathrm{mM}$ SCFA treatment. This is an interesting result, since the wild type strain experienced diminished attachment in the SCFA treatments. This data was unprecedented since there is a rather comprehensive body of literature on the regulatory elements of Ler on the LEE PAI. ${ }^{42,47,65,66,141,195,199,50,51,53,54,58,59,61,63} \mathrm{In}$ one research study, the ler gene was deketed from a rabbit EPEC (rEPEC) strain, and evaluated the behavior of this mutant in vivo. ${ }^{203}$ The results demonstrated a decrease of secreted LEEencoded proteins, and abolishment of rEPEC $\Delta l e r$ to cause disease in rabbits. ${ }^{203}$ The data presented in this manuscript suggests that Ler may not be the only regulator of the LEE2-LEE5 operons. Further investigation in alternative LEE regulators is necessary to understand this data.

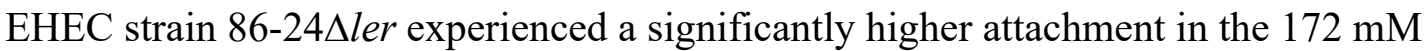
SCFA treatment compared to the $30 \mathrm{mM}$ SCFA treatment. This could be indicative of ler exerting negative control on expression of adhesins under higher concentrations of SCFAs. The

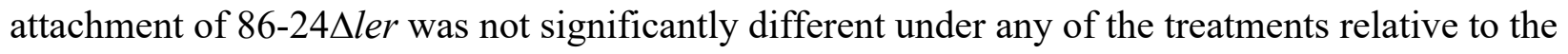
WT LB control. This trend is inconsistent with the observations in the GFP reporter assays, discussed in the section below. A reason for this could be that data in the GFP assay was 
generated with the 86-24_espA mutant strain and was looking at the esp promoter activity, not attachment. Previous research suggests that transcription of the LEE PAI is "largely" orchestrated by Ler. ${ }^{45,173,202,194}$ Yet the data generated in this study does not align with the literature data on Ler is the sole regulator of the LEE. A possible explanation for this phenomenon could be that an unknown alternative pathway may have been activated by the bacteria to replace function of Ler in the extreme case that the bacterium is missing the ler coding region. This alternative pathway can thus activate LEE encoded virulence genes necessary for attachment. It is conceivable for this mysterious cascade of events to generate side effects on the bacteria, such as providing EHEC with a protective effect from SCFA-induced toxicity. Although research has established that Ler is required for the activation of LEE1$5,{ }^{173,168}$ it was also shown that at high concentrations Ler is considered to be repressive of $L E E 1$ in EPEC. ${ }^{204,205}$ One study demonstrated that LEE1 expression can be stimulated by a prophageassociated protein RgdR in the absence of ler. ${ }^{206}$ This phage-associated protein RgdR could be the reason that attachment is not diminished in the ler mutant strain and specifically under the SCFA treatments. Transcriptional regulation of ler is exceptionally sophisticated. ${ }^{207}$ This fact is demonstrated simply by the unusually long upstream leader sequence of $L E E 1$ which is $\sim 170$ bases. ${ }^{201}$ What's more is that it is plausible that the strain of EHEC O157:H7 is continually evolving, perhaps a horizontally acquired gene may be the culprit to this behavior. Lastly, it is known that gene deletions in bacteria can potential alter the morphology of the cells. ${ }^{208}$ The attachment profile observed in $86-24 \Delta l e r$ can be attributed to the fact that the individual bacterial cells were larger compared to the wild type, particularly when it comes to the SCFA treatment conditions. More research in this realm is necessary. 


\subsection{EspA Protein Levels Remain Constant Across SCFA Treatments}

Western blot analysis of EspA protein production by EHEC O157:H7 strain 86-24 revealed no significant variability across treatment groups. The reason there was no statistically significant difference between treatments could be due to the EspA antibody used. There was a significant amount of troubleshooting involved in generating useful blots. The monoclonal EspA antibody was reconstituted in $25-50 \%$ glycerol or ethylene glycol, stored at minus $20^{\circ} \mathrm{C}$ and produced in 2009. According to a publication on antibody shelf life, an antibody stored in glycerol at minus 20, that has endured freeze thaw cycles has a typical shelf life of 1 year. ${ }^{209}$ That being said, the antibody generated bands for the LG DMEM condition as well as the 172 $\mathrm{mM} \mathrm{NaCl}$ condition. This is good evidence to suggest that protein level changes in EspA may not be influenced by SCFA treatment. There is no previously published data on the effect of SCFA on EspA protein levels. That being said, no conclusions can be drawn from this data.

\subsection{Modulation of esp Promoter Activity by Selected Culture Conditions}

EHEC gene espADB transcription was suggested to be associated with the promoter esp found at the extremity of the $\operatorname{sep} L$ coding region, just before the esp $A$ gene origin, while $\operatorname{sep} L$ was thought to be transcribed independently. ${ }^{165,167,168}$ This was the research upon which the experiments in this study, testing the promoter activity of esp $A$ in EHEC O157:H7 strain 86-24, were based on. The esp promoter was tested for its activity under a variety of conditions, and the results can be viewed in the results section of this manuscript. Chromosomal lacZ transcriptional fusion experiments assessing esp promoter activity in various EHEC strains failed to detect activity in the esp promoter. ${ }^{169}$ Within the same study, Northern blot analysis detected a $3 \mathrm{~kb}$ 
transcript for espA and $\operatorname{esp} D$. The authors who made this discovery proposed the possibility of an upstream promoter located at the beginning of the $\operatorname{sep} L$ coding region that may be responsible for the transcription of the $L E E 4$ operon, in which case espADB mRNA would be generated through post-transcriptional processing in EHEC. Further research into this matter has proven through the use of RT-PCR, Northern blot, Ribonuclease Protection Assay (RPA), and 5' and 3' Rapid Amplification of $\underline{\mathrm{c} D N A}$ Ends (RACE) that espADB mRNA is generated through posttranscriptional processing of the LEE4 operon in EHEC, all the while proving that $s e p L$ is a gene that belongs to the LEE4 operon. ${ }^{170}$ These researchers have shown that LEE4 processing occurs in an E. coli K-12 background. RNase E, the molecule fundamental to RNA metabolism with roles in degradation, processing and maturation of various RNA molecules is responsible for the cleavage of LEE4 into the subsequent gene products. ${ }^{170}$ These findings have critical ramifications on the realm of T3SS regulation, SepL and EspADB gene expression. The consequences of the findings mean that $\operatorname{sep} L$ promoter activity is the true measure of how much $\operatorname{esp} A$ gene is being transcribed, whereas the esp promoter activity is much lower.

\subsubsection{Impact of Low Glucose DMEM Culture on esp Promoter Activity}

The esp promoter, that precedes the espA gene was tested under the Low Glucose (LG)

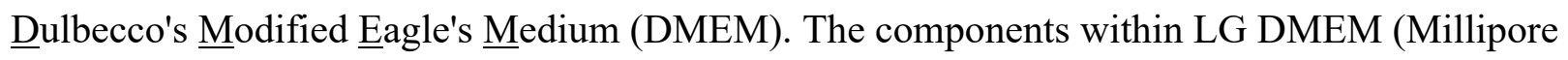
Sigma D6046) include a variety of inorganic salts including sodium bicarbonate, 15 amino acids in different concentrations, 9 vitamins, D-glucose at a concentration of $1 \mathrm{~g} / \mathrm{L}$, phenol red, and pyruvic acid. Any or all of these components may have an effect on the activity of the esp promoter. The addition of low concentration (30 mM) SCFA mix to the LG DMEM was intended to mimic the SCFA concentration of the small intestine while the higher concentration 
(172 mM) SCFA mix was intended to mimic the SCFA concentration of the colon. The colon is the site at which EHEC O157:H7 establishes infection.

EHEC O157:H7 strain 86-24_espA expression of GFP labelled esp promoter on pCR2.1TOPO was assessed under LG DMEM alone, as well as in combination with $30 \mathrm{mM}$ SCFA and $172 \mathrm{mM}$ SCFA. Research in this realm has shown that EspA protein production, and attachment of EHEC is significantly diminished under oxygenated conditions relative to microaerobic conditions. ${ }^{210}$ The data in this study shows that the activity of the esp promoter is not affected by oxygen availability other than in combination with biotin. It is possible that the regulation of EspA production is occurring at the translational level. Especially when considering that LEE4 is post-transcriptionally processed into the corresponding proteins. Furthermore, there were no changes during the stationary phase amid the various treatment groups based on GFP fluorescence. Not only were the treatment groups the same, the expression of esp was significantly diminished compared to the exponential phase expression. The diminished activity of this promoter during exponential phase can be due to one of two reasons. The first reason being due to the bacterial growth having reached or surpassed a milestone in growth. The milestone being a turning point where rather than the bacteria continuing to divide and make new proteins, the bacteria begin to die off due to the overwhelming amount of waste products in the media. When the bacteria are not actively dividing, they would not be actively producing proteins, especially proteins that are associated with virulence such as EspA. The second reason would have to assume the bacteria are continuing to divide and in a healthy state. The reason esp activity would diminish in such a state would be due to the fact that alternative regulatory pathways are activated during exponential phase, that would not be active during stationary 
phase. Those regulatory pathways may potentially act to repress the LEE PAI, or at least repress the LEE4 operon.

During the exponential phase of growth, there was a significant increase in the activity of $e s p$ with the addition of $30 \mathrm{mM}$ SCFA compared to LG DMEM alone. The addition of $172 \mathrm{mM}$ SCFA to LG DMEM elicited approximately a three-fold increase in esp activity compared to the LG DMEM alone, and 1.3-fold increase compared to the $30 \mathrm{mM}$ SCFA condition. Despite the growth having been reduced by the presence of the $172 \mathrm{mM} \mathrm{SCFA}$, the increase in expression is still significant. This data suggests that SCFA are important signalling molecules that may play an important role in signaling location along the GI tract. The high $172 \mathrm{mM}$ SCFA concentration is formulated to mimic that of the colon, the site at which EHEC O157:H7 colonizes the human gut. $^{211}$

Removal of oxygen from the culture media by the addition of mineral oil (MO) overlay had a similar effect to the LG DMEM in full oxygen. The increase between LG DMEM and the two SCFA conditions mirror perfectly the fold increase observed in the full oxygen condition. For this reason, it appears as though esp promoter activity is not dependent on oxygen availability. Evidence of an up-regulation of a promoter (esp) that precedes an important virulence gene $(\operatorname{esp} A)$ known to be necessary for successful infection proves that short chain fatty acids are important players in mediating EHEC infection response.

\subsubsection{Impact of High Glucose DMEM Culture on esp Promoter Activity}

EHEC O157:H7 86-24_espA promoter activity of the esp promoter was tested under high glucose (HG) DMEM condition with high oxygen and low oxygen. The expression of esp in the high oxygen condition during stationary phase showed highest expression when the bacteria were exposed to $172 \mathrm{mM}$ SCFA, the expression was nearly 3.4-fold compared to HG DMEM 
alone. Lower expression was observed at $30 \mathrm{mM}$ SCFA compared to $172 \mathrm{mM}$ SCFA, while it was still 1.3-fold higher than HG DMEM alone. Therefore, large intestinal concentration of SCFA appears to be an important environmental cue for esp promoter activity. The activity appears to be independent of glucose concentration since the increase in activity mirrors that observed in LG DMEM. Further, the stationary phase displays no change in activity and overall much lower activity, just as in the LG DMEM condition.

When oxygen was restricted within the media through the use of $\mathrm{MO}$, the trend was similar as in the high oxygen condition. The $30 \mathrm{mM}$ SCFA was not significantly different from the HG DMEM alone, while 172mM SCFA condition showed a significantly elevated activity. The expression of esp in stationary phase was significantly lower than exponential phase in both the low oxygen and high oxygen condition, furthermore there was no change detected among the various treatments in either high oxygen or low oxygen condition. There are a handful of publications assessing the activity of the esp promoter using the $\beta$-galactosidase assay. ${ }^{165,167,168}$ These publications did not analyze any of the conditions tested within this study.

\subsubsection{Effect of Magnesium Concentration on esp Promoter Activity}

EHEC O157:H7 esp promoter activity was assessed under the influence of M9 minimal media in the low magnesium $\left(\mathrm{Mg}^{2+}\right)$ condition and high $\mathrm{Mg}^{2+}$ condition. Magnesium is an important cofactor for many enzymes to function properly within the human body. Humans must obtain magnesium from the environment through their diet. ${ }^{212}$ The intestinal absorption of $\mathrm{Mg}^{2+}$ occurs predominantly in the small intestine - particularly the distal jejunum and ileum. ${ }^{213}$ For this reason, it is believed that the greater $\mathrm{Mg}^{2+}$ concentration would be found in the small intestine. That being said, $\mathrm{Mg}^{2+}$ status is highly dependent on the body's need for $\mathrm{Mg}^{2+}$. There are a variety of endogenous and exogenous factors that affect the absorption of magnesium in the 
GI tract. For one, if the human body contains sufficient $\mathrm{Mg}^{2+}$, absorption would not occur, leading to higher $\mathrm{Mg}^{2+}$ concentration along the length of the GI tract. ${ }^{212}$ The low $\mathrm{Mg}^{2+}$ condition would be representative of the condition under which the PhoP-PhoQ two-component regulatory sensor kinase would be activated in EHEC, whereas the high $\mathrm{Mg}^{2+}$ condition would not activate said two-component regulatory system.

Research in Edwardsiella tarda (E. tarda) showed that the native PhoP-PhoQ system is capable of sensing temperature and $\mathrm{Mg}^{2+}$ and regulating T3SS genes through esr $B$ activation. ${ }^{214,215}$ Due to this evidence, this study sought to test whether EHEC PhoP-PhoQ sensor kinase happens to regulate downstream T3SS genes. If the PhoP-PhoQ two component system was involved in T3SS gene regulation, it is expected that the esp promoter activity data would differ between the low $\mathrm{Mg}^{2+}$ and the high $\mathrm{Mg}^{2+}$ experiments. This was in fact not the case, the data between the low $\mathrm{Mg}^{2+}$ and high $\mathrm{Mg}^{2+}$ were essentially identical. The differences among treatment groups for both low $\mathrm{Mg}^{2+}$ and high $\mathrm{Mg}^{2+}$ were interesting. During exponential phase, the highest expression was yet again observed under the $172 \mathrm{mM}$ SCFA condition in both low and high $\mathrm{Mg}^{2+}$. Interestingly the trend flipped in the stationary phase, where the expression of esp promoter was higher in M9 Low/High $\mathrm{Mg}^{2+}$ alone and in the M9 Low/High $\mathrm{Mg}^{2+}$ with 30 mM SCFA. The expression was significantly higher than the expression observed in the exponential phase by the $172 \mathrm{mM}$ SCFA treatment. This is interesting data as the previous data showed no expression in stationary phase, let alone a flip in expression such as this one. That being said, overall expression of this promoter is much lower than the previous experiments done in LG DMEM and HG DMEM. This flip in expression from exponential to stationary phase is likely owing to the fact that the bacteria grow and divide much slower in M9 minimal media due to the nutrient restriction. ${ }^{216}$ 


\subsubsection{Reactive Oxygen Species Influence on esp Promoter Activity}

EHEC O157:H7 esp promoter activity was assessed in the presence of hydrogen peroxide $\left(\mathrm{H}_{2} \mathrm{O}_{2}\right)$. The activity of the esp promoter was highest in exponential phase when the bacteria were grown in HG DMEM. The activity of esp in HG DMEM was significantly higher compared to the bacteria grown in LG DMEM. Furthermore, the activity was significantly lower in LB than it was in LG DMEM and HG DMEM. Stationary phase brought on very low activity in the esp promoter with no significant differences between the various culture media. It appears as though the presence of ROS such as $\mathrm{H}_{2} \mathrm{O}_{2}$ have an up-regulatory effect on the esp promoter in the presence of high glucose. There is no data in the literature that has studied the effect of this combination of molecules on EHEC.

\subsubsection{Biotin Status Modulates esp Promoter in Low Oxygen}

The influence of biotin was tested to assess the activity of esp under biotin exposure. Research has shown that high biotin concentrations $(>50 \mathrm{nM})$ have a repressive effect on T3SS genes. ${ }^{146-148,150}$ This is thought to be due to the fact that high biotin concentrations would be found in the small intestine (where EHEC does not colonize), while lower biotin concentrations would be found in the large intestine (the site of EHEC of colonization). ${ }^{146-148,150}$ This research concluded that biotin, much like SCFA, is an important molecular cue that would allow EHEC to be spatially aware of its whereabouts along the GI tract. The activity of the esp promoter as influenced by the presence of $30 \mathrm{nM}$ biotin in LG DMEM was significantly lower in the high oxygen condition compared to the low oxygen condition during exponential phase. Furthermore, that activity was significantly higher than the activity of the esp promoter in LG DMEM alone, and LG DMEM MO. This makes sense as the lower oxygen concentrations would be found in the colon, where the oxygen concentrations in the lumen can be as low as 0.1 to $1 \mathrm{~mm} \mathrm{Hg.}{ }^{217} \mathrm{In}$ 
the biotin experiments, the activity of the esp promoter was higher in the low oxygen condition compared to the high oxygen condition in stationary phase as well. This data suggests that oxygen plays an important role in the activity of the esp promoter, particularly in combination with biotin. Biotin appears to be both a positive and negative modulator of the LEE as in high concentrations, biotin leads to LEE repression ${ }^{150}$, yet in low concentrations in combination with low oxygen, biotin leads to esp promoter activation.

\subsection{Conclusions}

Based on the evidence provided in this study, it is conceivable to ascertain that short chain fatty acids (SCFAs) play a meaningful function in EHEC location sensing. The promoter of an important virulence gene was demonstrated to become active when exposed to SCFA concentrations that mimic that of the large intestine. Moreover, the data from this study has revealed the importance of oxygen and biotin concentration in the activity of the esp promoter.

The overall hypothesis of this research stated as follows: EHEC exposure to large intestinal micro environmental conditions up-regulates the expression of LEE-encoded virulence factors that promote the formation of $\mathrm{A} / \mathrm{E}$ lesions at the site of infection. The experiments conducted throughout this study supported the hypothesis by showing that LEE-encoded promoter esp has higher activity in the environment that mimics its site of infection, the colon.

The first objective was to generate transcriptional/translational GFP reporter fusions for the genes eae, espA and ler in EHEC 86-24. This objective was met through employing the new method of CRISPR-Cas9 mutagenesis.

The second objective was to assess expression of the GFP reporter in each of the reporter fusions in EHEC 86-24 after treatment with each of the following treatments: 30mM SCFA, 172mM SCFA, 30mM NaCl, 172mM NaCl, 30mM SCFA low oxygen, 172mM SCFA low 
oxygen, $30 \mathrm{mM} \mathrm{NaCl}$ low oxygen, $172 \mathrm{mM} \mathrm{NaCl}$ low oxygen and other relevant conditions that are representative of small molecules found in both the large and small intestine. These experiments elucidated the importance of SCFA in the regulation of LEE encoded genes. The third objective set out to assess and compare attachment of EHEC 86-24 under large intestinal conditions. The objective was met and the data generated showed that espA is a critical gene for EHEC to attach to epithelial cells.

EHEC O157:H7 is an important pathogen that is currently at the center of an outbreak associated with romaine lettuce. ${ }^{218}$ This outbreak, as with many previous incidents of illness underscore the importance of further building our understanding of this bacterial pathogen's virulence profile. Through exposing the pathways, regulatory proteins, and genes implicated in the bacterial arsenal for establishing infection, researchers could begin to test drugs and other compounds that may potentially inhibit these targets. By attacking the bacterial virulence proteins, rather than vital division pathways, bacterial survival is not threatened. If the bacterial survival is not under threat, the organism possesses less agency to evolve mechanisms to overcome these hurdles leading to a reduction in drug resistance. The hope is that this, and future studies of this nature, will encourage and inform the development of more adequate antimicrobials focused on disrupting the regulation of virulence genes, and ultimately EHEC pathogenesis.

\subsection{Future Directions}

The next logical steps to pursue, with regard to environmental cues that affect LEE virulence genes, would be to examine the two-component systems (TCSs) that regulate the expression of LEE. In particular, it would be interesting to identify the TCS by which EHEC is able to sense SCFAs from the environment. It would be fascinating to discover the 
conformational change that might occur in this unknown TCS when EHEC is in a higher concentration of SCFA that leads to an activation of T3SS genes, if any. Several TCSs have been identified as affecting LEE regulation. For instance, QseCB and QseEF activate LEE by sensing AI-3, adrenaline and noradrenaline. The challenge would be to associate SCFA detection to either an existing LEE affiliated TCS, or discover an entirely new TCS through which EHEC is capable of sensing SCFA concentrations in the environment.

When it comes to biotin, researchers have shown that biotin binds to BirA, which activates the fur promoter, leading to translation of the Fur protein, where Fur through an unknown mediator represses LEE expression. ${ }^{147}$ One direction would be the undertaking the discovery of an unidentified LEE regulator that represses LEE expression directly due to biotin concentration. 


\section{APPENDICES}

Appendix A-CRIPSR/Cas9 Mutagenesis of EHEC O157:H7

\subsection{CRISPR/Cas9 Mutagenesis of EHEC O157:H7}

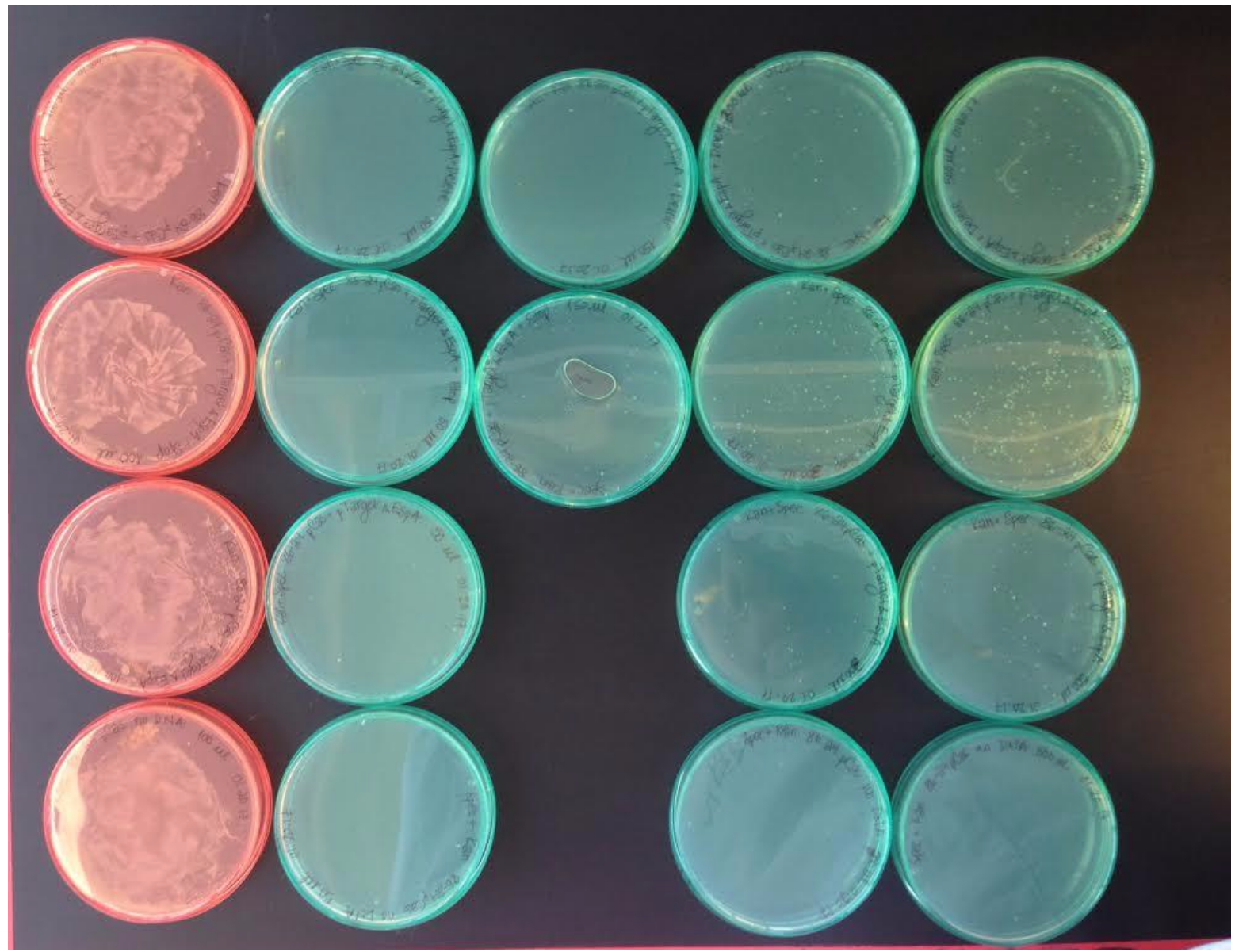

\section{Figure 18. EHEC 86-24 $\Delta \operatorname{esp} A$ mutants}

$*$ Red plates $=$ Kanamycin $(50 \mu \mathrm{g} / \mathrm{ml})$

$*$ Green plates $=$ Kanamycin $(50 \mu \mathrm{g} / \mathrm{ml})+$ Spectinomycin $(50 \mu \mathrm{g} / \mathrm{ml})$

The red plates are a control to see the rate of bacteria killed from the electroporation event. None

of the plates displayed low numbers suggesting that electroporation did not damage the

population viability. Various dilutions were plated on double selection kanamycin/spectinomycin plates displayed highest growth for the stop oligomer insertion with decreasing numbers for the deletion oligomer insertion. There was no growth on the plates that did not receive any pTarget $\triangle \operatorname{esp} A$ or oligomers. 


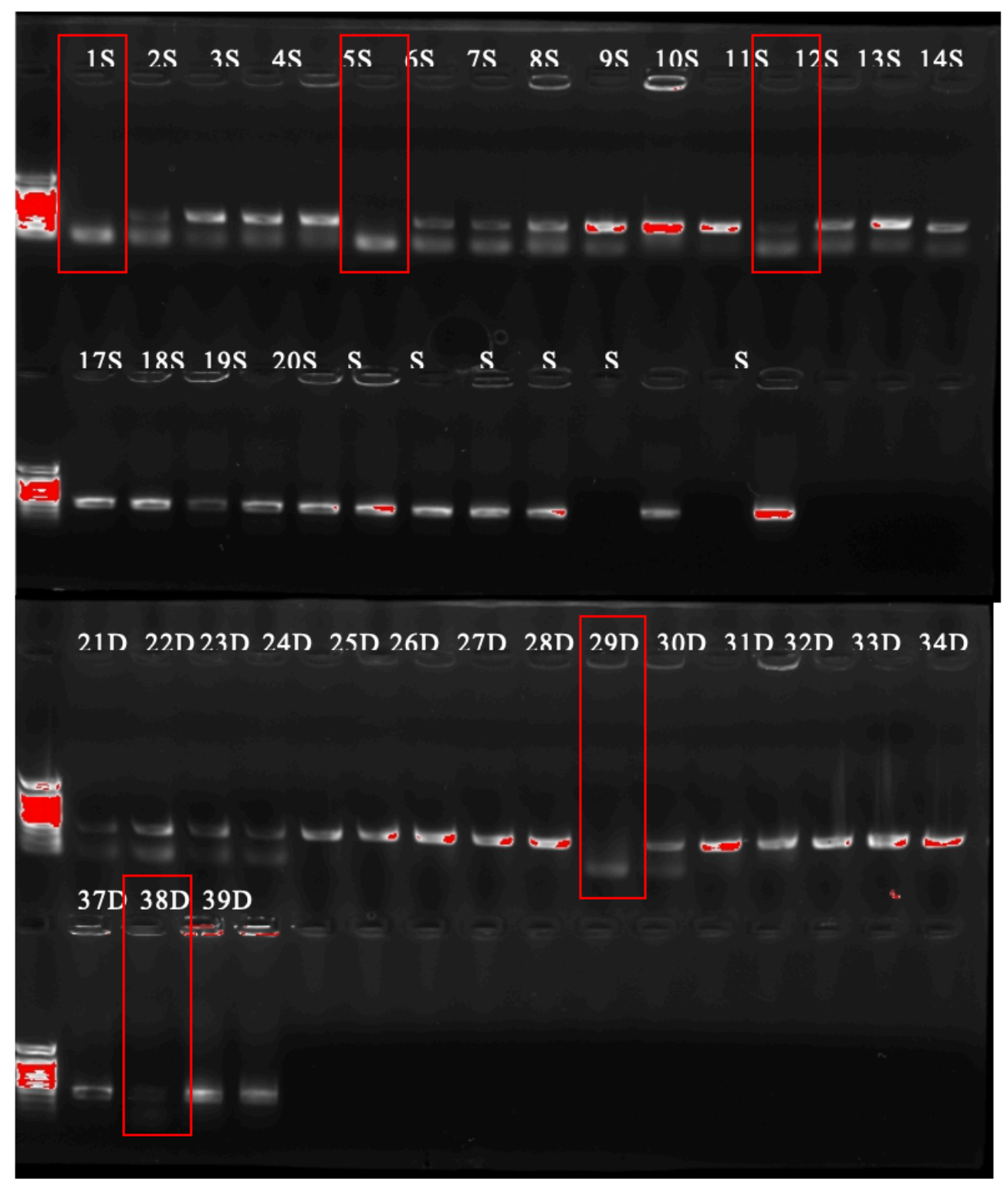

\section{Figure 19. Colony PCR of Stop and Delete espA Mutants}

GeneRuler ${ }^{\mathrm{TM}} 1 \mathrm{kB}$ Plus was used in the left lanes and $5 \mu \mathrm{l}$ of the products from the colony PCR were loaded into the wells of the gel. Several colonies (Red box) were selected for size shift analysis by PCR using the size shift primers (Table 2). All the selected colonies were confirmed to be stop mutants and delete mutants respectively. 


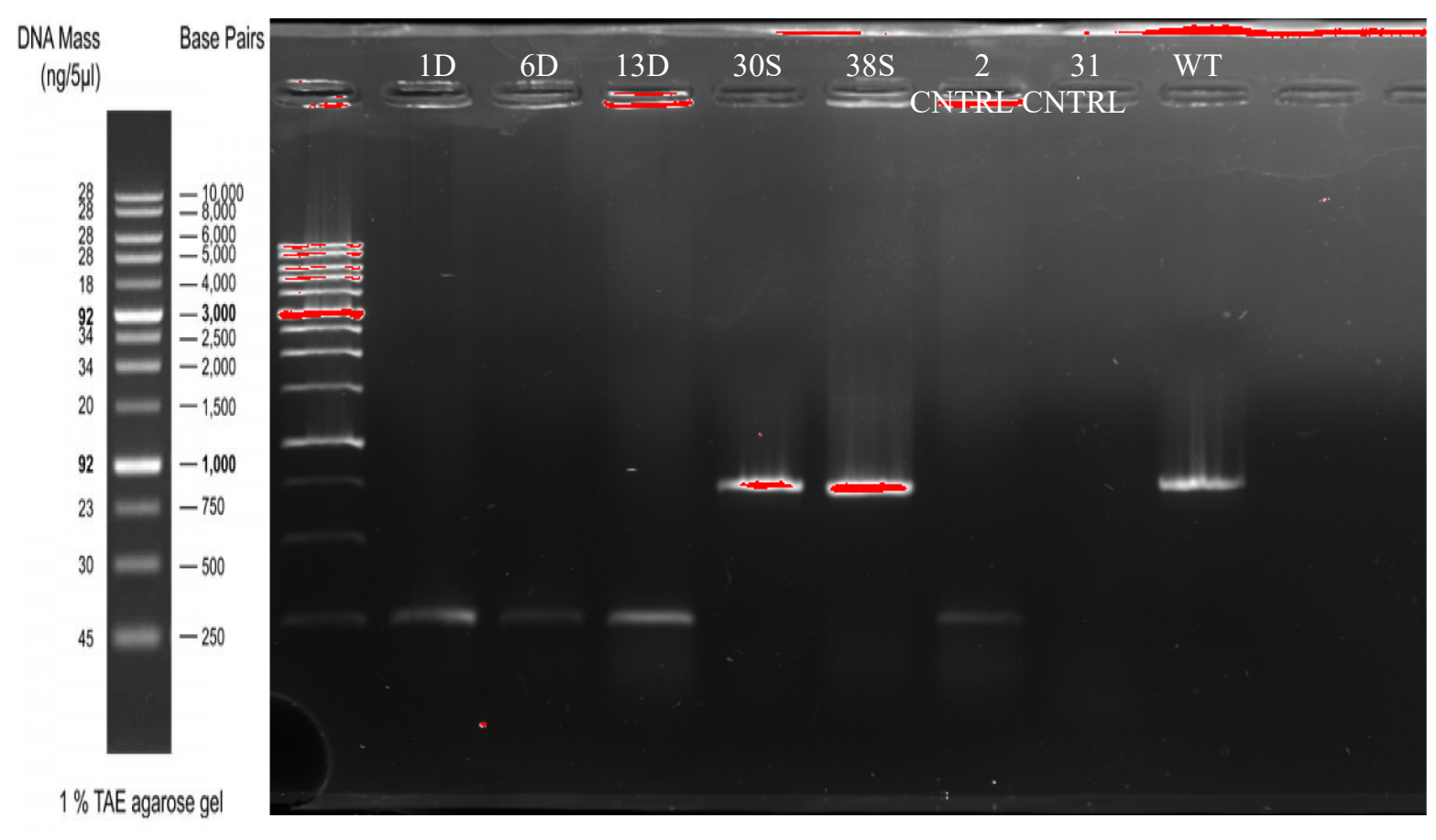

Figure 20. Mutant Screening using size shift oligomers

Using the size shift oligomers from table 2, it was possible to see that the stop codon mutants contained the same esp $A$ gene size as wild type 86-24 while the delete mutants contained a smaller gene product. The expected gene product for the delete was 256 base pairs, considering it is appearing slightly above the 250 base pair band of the $1 \mathrm{~kb}$ FroggaBio DNA ladder, it was convincing that the deletion mutation took place. 


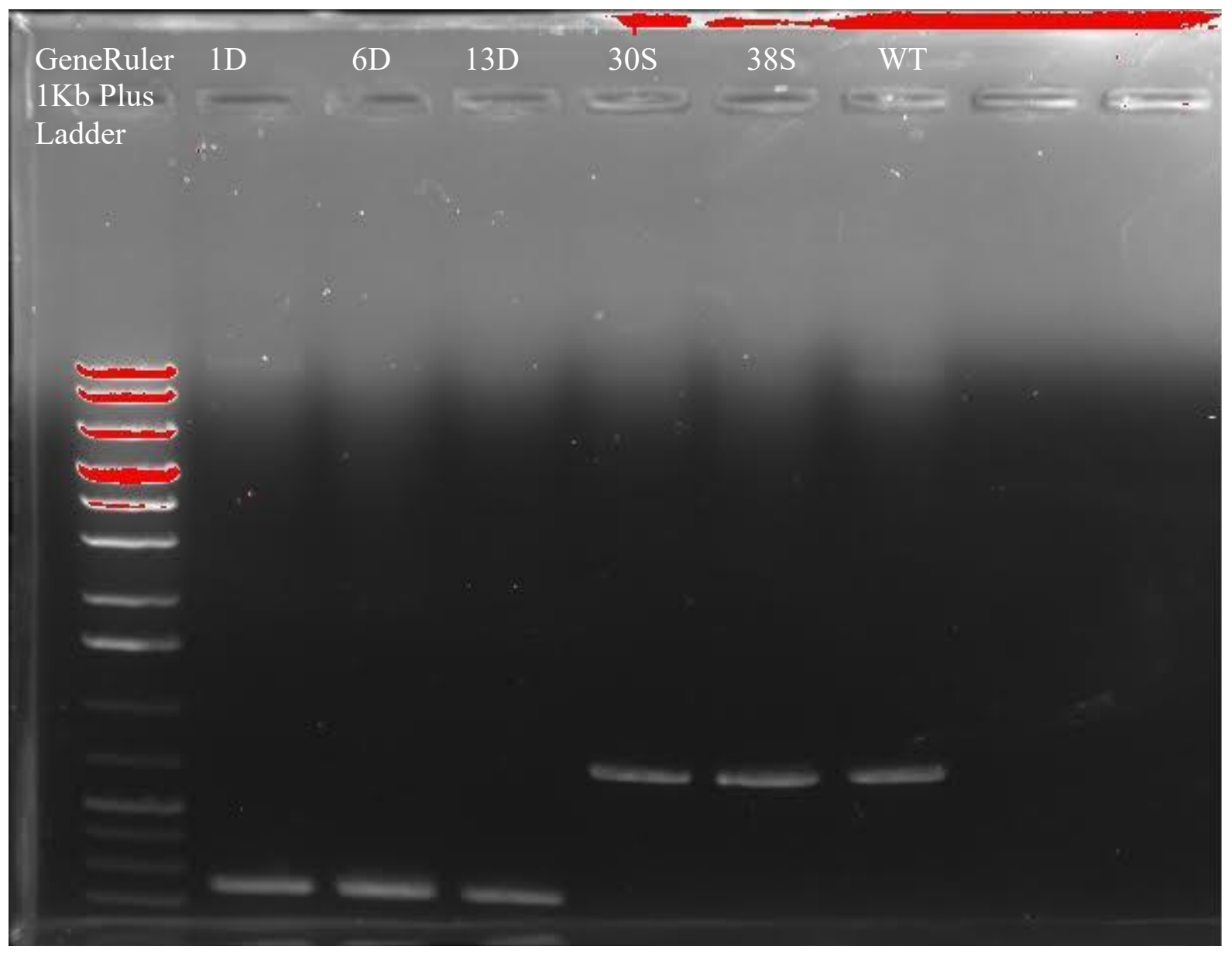

\section{Figure 21. Mutant Screening using size shift oligomers}

Using the size shift oligomers from table 2, it was possible to see that the stop codon mutants contained the same esp $A$ gene size as wild type 86-24 while the delete mutants contained a smaller gene product. The expected gene product for the delete was 256 base pairs, considering it is appearing slightly above the 250 base pair band of the $1 \mathrm{~kb}$ FroggaBio DNA ladder, it was convincing that the deletion mutation took place. 


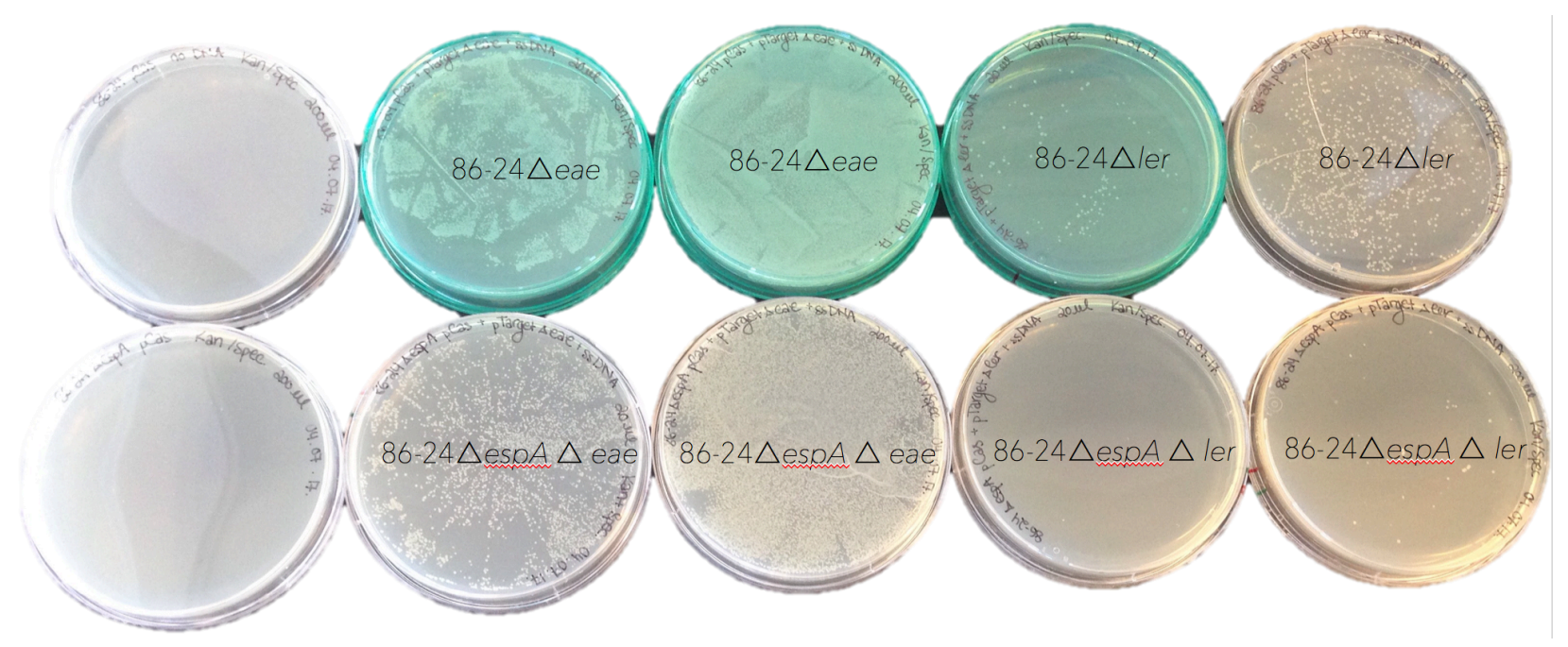

Figure 22. Potential deletion mutant colonies on selective plates in genes eae and ler Arabinose was used to induce the $\lambda$-Red system in pCas containing EHEC 86-24 WT cells and

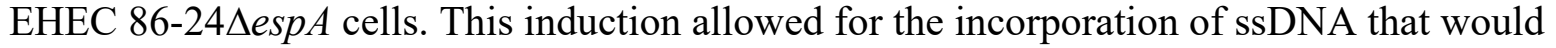
delete significant portions of the genes eae and ler in WT EHEC and EHECAespA. The colonies that appear on the double selection plates $(50 \mu \mathrm{g} / \mathrm{ml}$ kanamycin \& $50 \mu \mathrm{g} / \mathrm{ml}$ spectinomycin) above

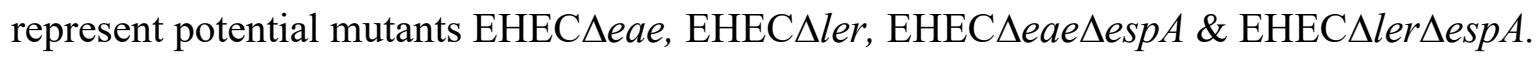




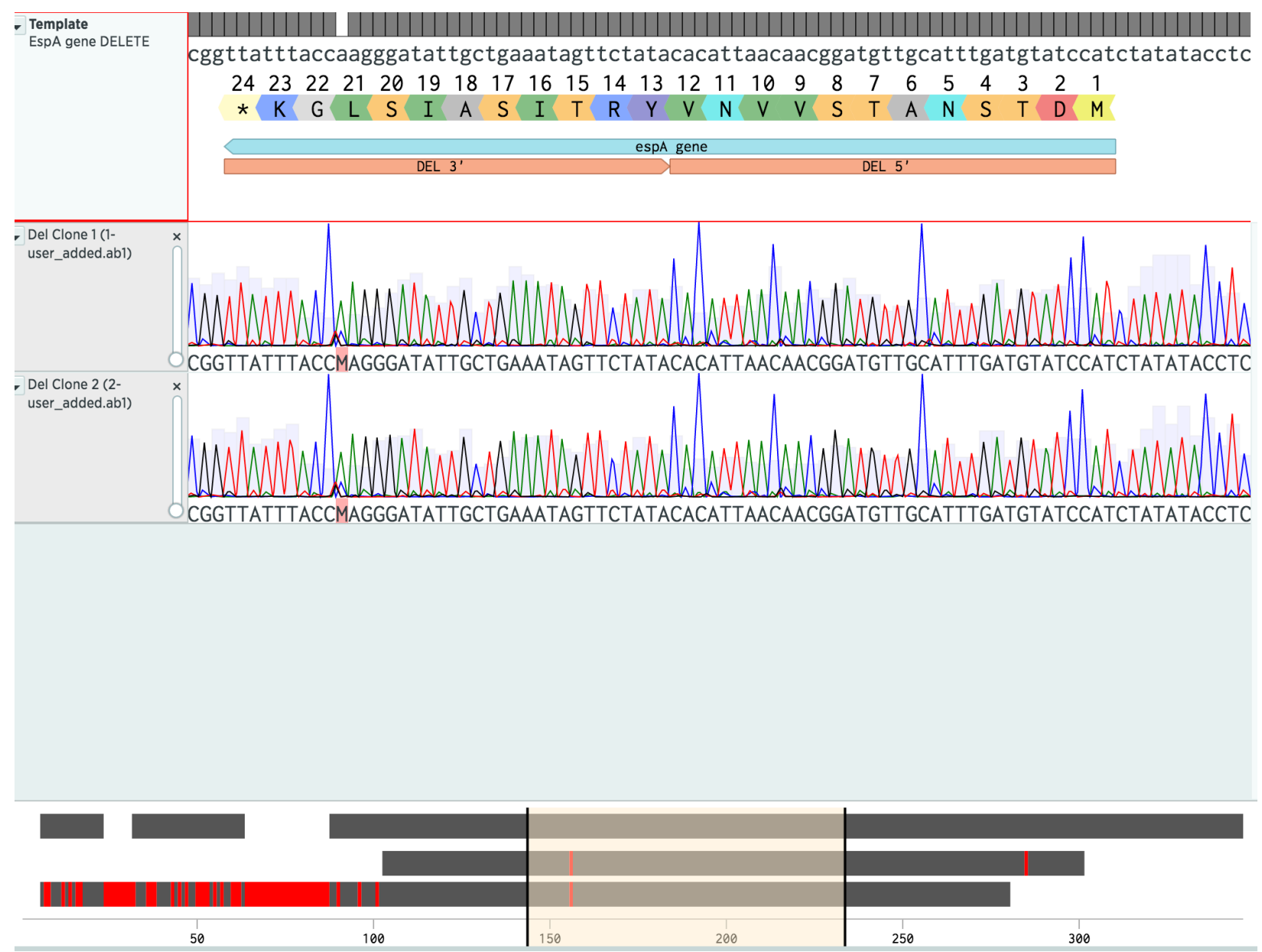

Figure 23. Sequencing Data of 86-24 $\Delta \operatorname{esp} A$ Deletion Mutant

The sequencing data of the forward and reverse strands of the purified PCR product using iProof High Fidelity polymerase including the size shift in the PCR product compared the wild type EHEC 86-24 clearly shows that the strain underwent a deletion in the esp $A$ gene region. This is conclusive data that the CRISPR/Cas9 mutagenesis protocol has been optimized and can be used to introduce other mutations within the pCas containing cells. The deletion will lead to the transcription of a truncated, dysfunctional version of the EspA protein subunit that makes up the needle portion of the T3SS, and subsequently lack of $\mathrm{A} / \mathrm{E}$ lesion formation or reduced formation of $\mathrm{A} / \mathrm{E}$ lesions. This mutant along with others will be used in infection assays that asses the ability for this bacterium to form $\mathrm{A} / \mathrm{E}$ lesions on Caco-2 intestinal cell lines. 


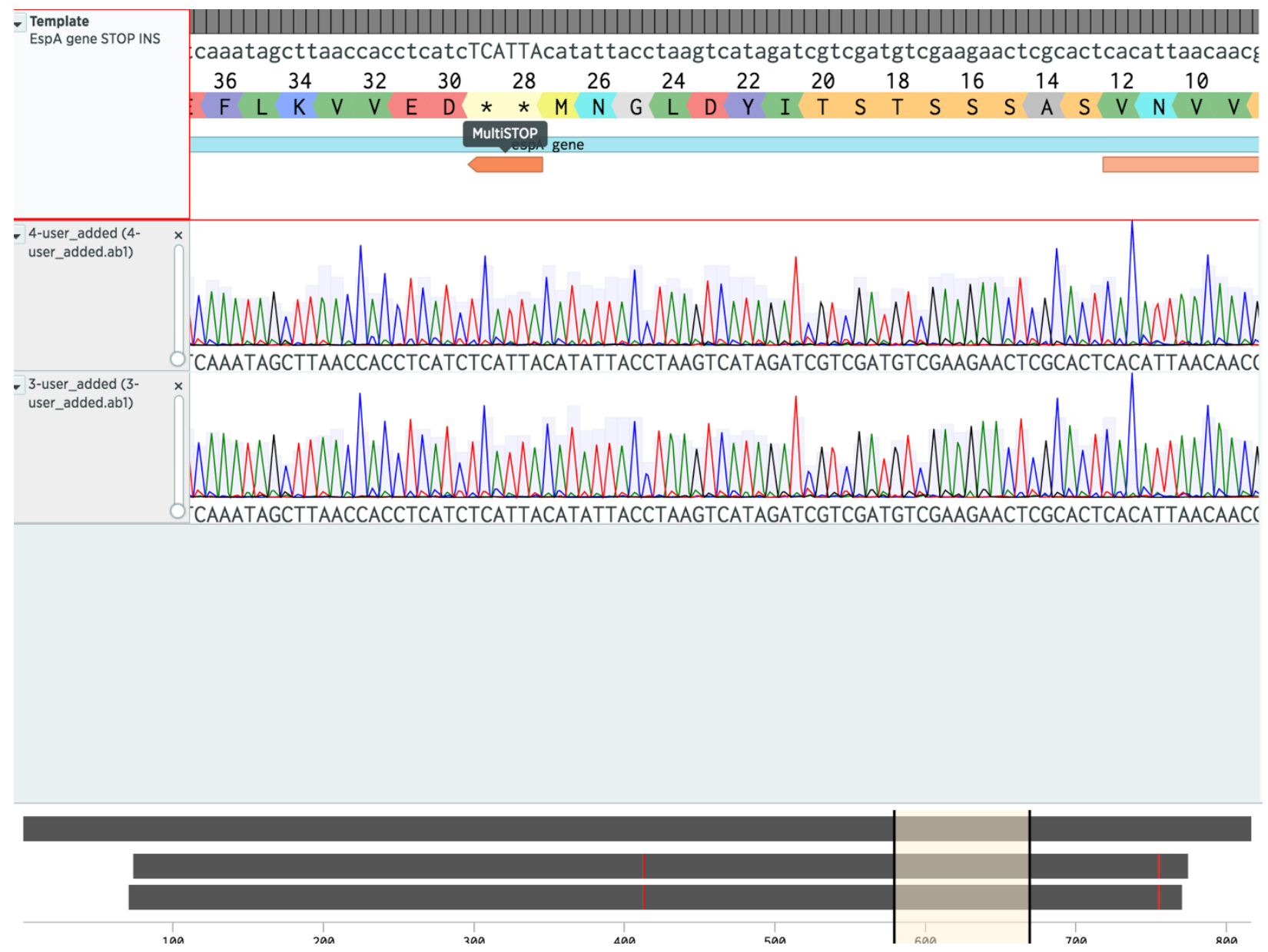

Figure 24.Sequencing Data for the EHEC 86-24 $\Delta e s p A$ stop codon insertion mutant

The sequencing data of the forward and reverse strands of the purified PCR product using iProof High Fidelity polymerase shows that the two stop codons were inserted in the exact position that the oligonucleotides were designed to insert this new DNA. This is conclusive data that the CRISPR/Cas9 mutagenesis protocol has been optimized and can be used to introduce other mutations within the pCas containing cells. This bacterium when attempting to transcribe the gene espA that encodes for the EspA protein subunit that comprises the needle portion of the T3SS will experience the ribosome to fall off the chromosomal DNA and will only be able to produce a truncated version of the EspA protein. This will lead to a lack of the needle in the $\mathrm{T} 3 \mathrm{SS}$, and potentially lack of $\mathrm{A} / \mathrm{E}$ lesion formation. 


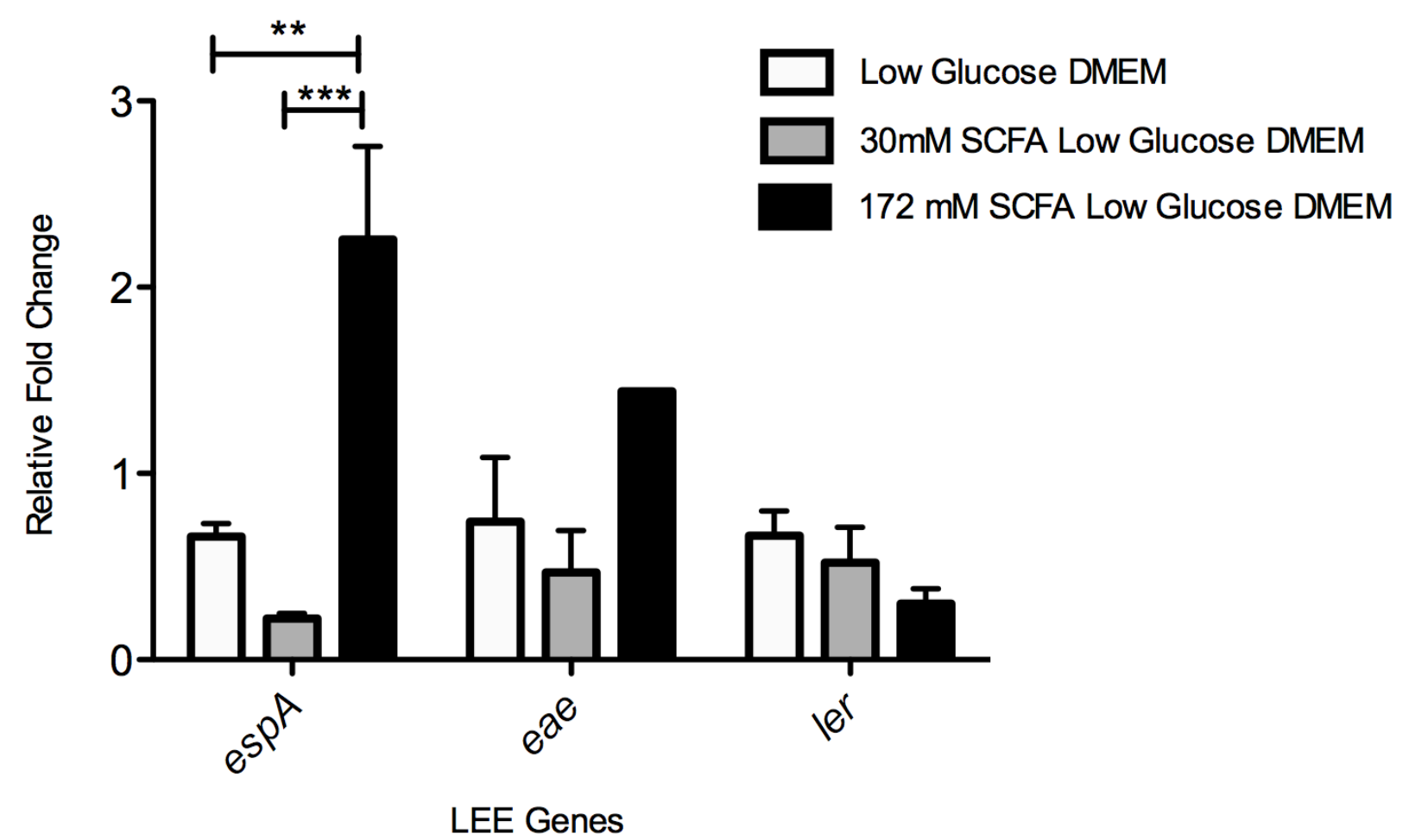

Figure 25. RT-qPCR Data Using 16SrRNA as the Housekeeping Gene

Compiled data from the assessed cDNA using 16SrRNA as the house keeping gene. The biological replicates are incomplete as the Cq yielded for the cDNA were large, in the high 20s, to low 30 s. 

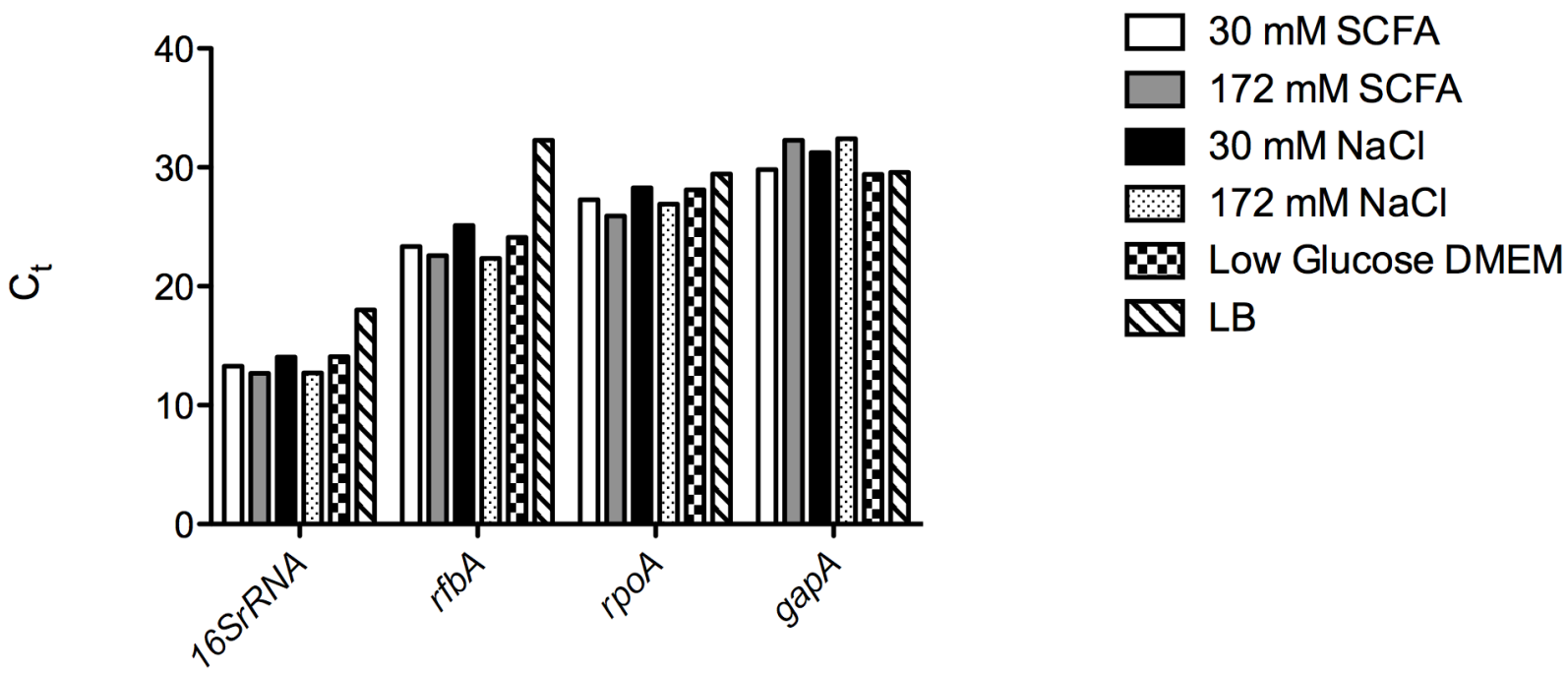

Housekeeping Genes

Figure 26. Raw $\mathrm{Ct}$ values testing the primers for various housekeeping genes in cDNA from EHEC 86-24 grown under varying conditions

Primers for the commonly used Escherichia coli housekeeping genes $16 \operatorname{SrRNA}, r f b A$, rpoA, and gapA were tested for efficiency and variation purposes on cDNA generated from purified RNA. The bacteria were grown under the varying conditions listed in the legend all in low glucose DMEM; 30mM SCFA, 172mM SCFA, 30mM NaCl, 172mM NaCl, low glucose DMEM, and LB. The primer set that showed lowest $\mathrm{Ct}$ values with little variation in expression across the various conditions was $16 \operatorname{Sr} R N A$, although this primer set showed a spike in expression under the LB condition, as $\operatorname{did} r f b A$. The primer set for $r p o A$ showed least variation, but the $\mathrm{Ct}$ values were rather high for a housekeeping gene, hovering at just below $30 \mathrm{Ct}$. Lastly, gapA showed some stability along the changing conditions but had the highest $\mathrm{Ct}$ values of all the primer set, hovering at just above and below $30 \mathrm{Ct}$, this is not ideal for a housekeeping gene. 


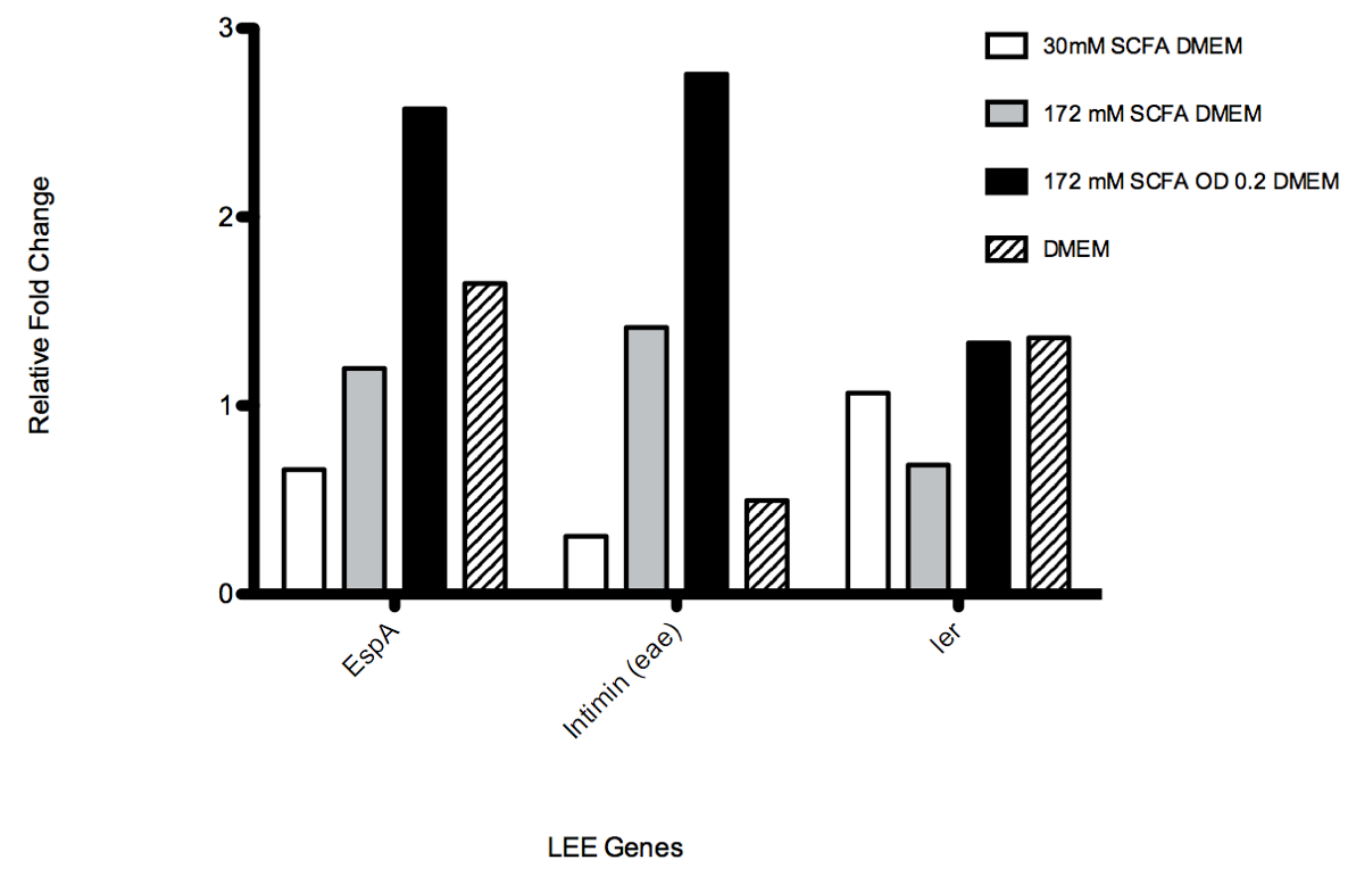

Figure 27. qRT-PCR of LEE gene expression under varying conditions

Reverse transcription quantitative polymerase chain reaction was used to determine expression of various LEE encoded genes. 


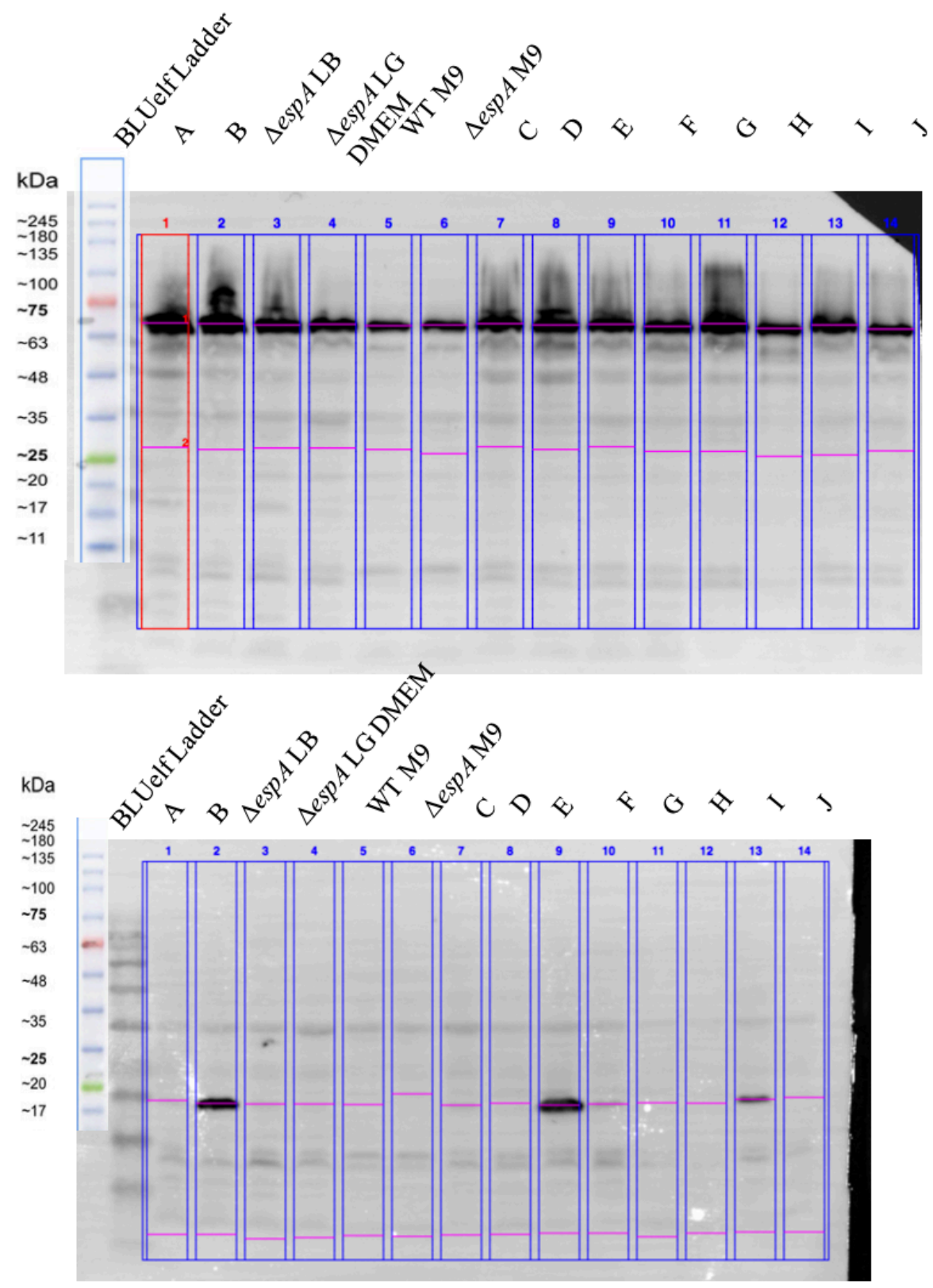

Figure 28. First biological replicate of EspA (25 kDa) expression under various conditions

The blot above is an anti-dnaK loading control for the blot below run on a discontinuous buffering system SDS-PAGE gel at $70 \mathrm{~V}$ for 30 minutes and $120 \mathrm{~V}$ for 1 hour and 30 minutes, or until the dye front reaches the bottom of the plate. The $\alpha$-EspA antibody was used at a concentration of 1:3000 while the $\alpha$-DnaK was used at a dilution of 1:10,000. 


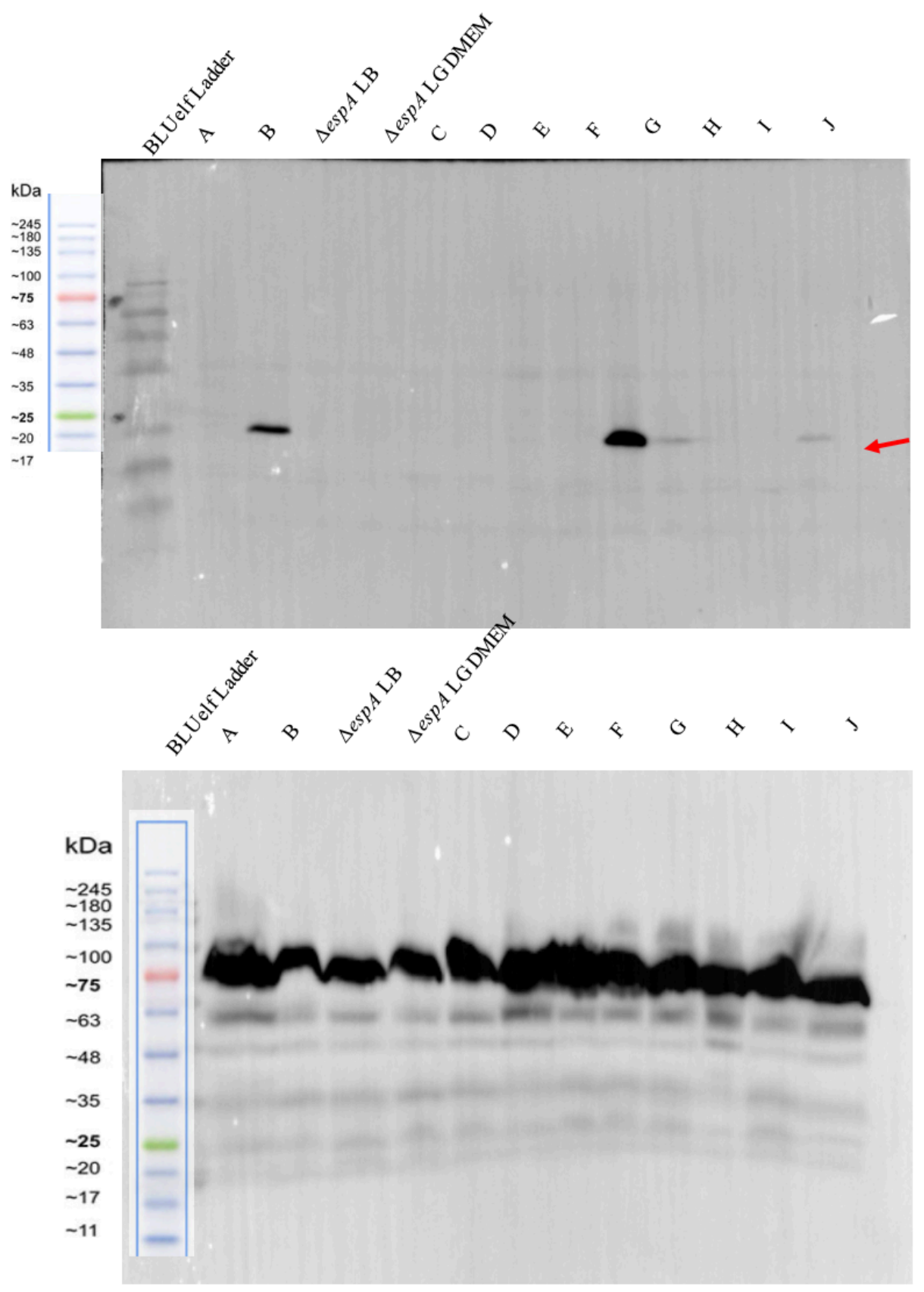

Figure 29. Second Biological replicate of EspA (25 kDa) expression under various conditions.

The blot above is an anti-dnaK loading control for the blot below run on a discontinuous buffering system SDS-PAGE gel at $70 \mathrm{~V}$ for 30 minutes and $120 \mathrm{~V}$ for 1 hour and 30 minutes, or until the dye front reaches the bottom of the plate. The $\alpha$-EspA antibody was used at a concentration of 1:3000 while the $\alpha$-DnaK was used at dilution of 1:10,000. 

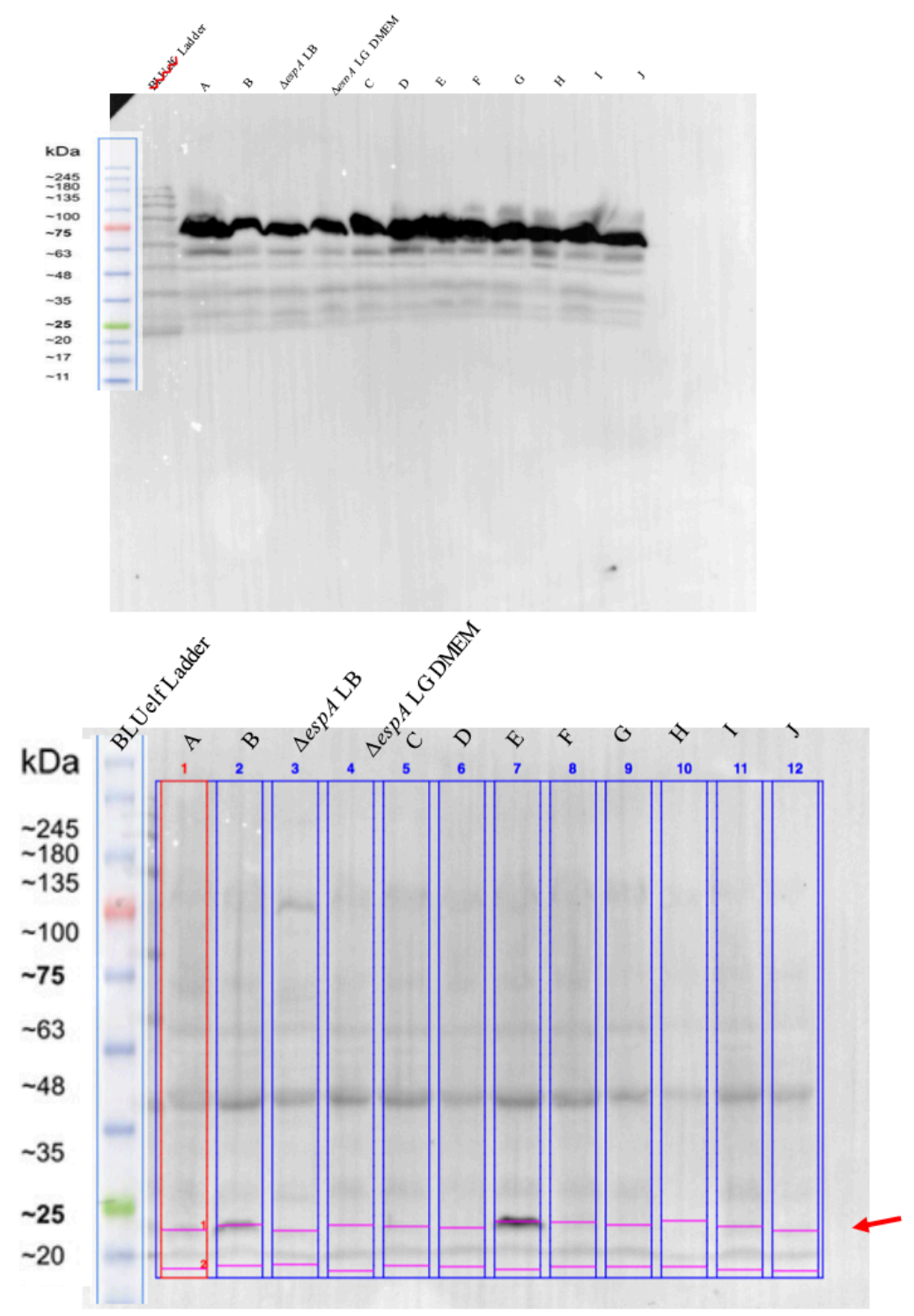

Figure 30. Third biological replicate of EspA ( $25 \mathrm{kDa})$ abundance in cell lysates under various conditions

The blot above is an anti-dnaK loading control for the blot below run on a discontinuous buffering system SDS-PAGE gel at $70 \mathrm{~V}$ for 30 minutes and $120 \mathrm{~V}$ for 1 hour and 30 minutes, or until the dye front reaches the bottom of the plate. The $\alpha$-EspA antibody was used at a concentration of 1:3000 while the $\alpha$-DnaK was used at a dilution of 1:10,000. 


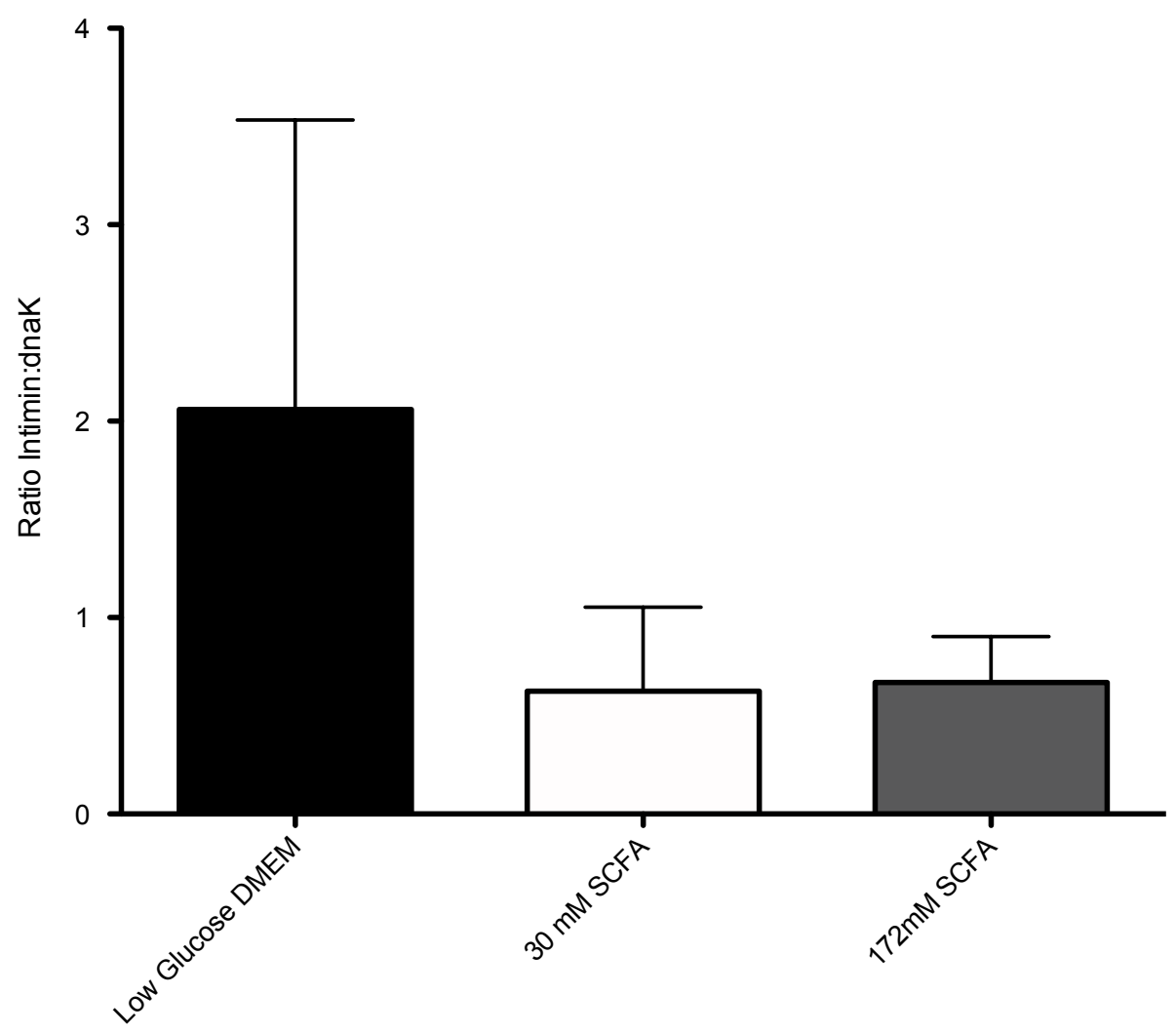

Figure 31. Intimin protein expression as analyzed through Western Blotting

The expression of transmembrane protein Intimin was analyzed with polyclonal anti-Intimin antibodies at a concentration of 1:5000. The data was normalized to the respective controls, in the case of LG DMEM, the data was normalized to LB, while in the case of the SCFA condition, the data was normalized to the corresponding concentrations of $\mathrm{NaCl}$. This data is generated from 3 biological replicates. Data are presented as means \pm s.d.; $n=3$. ${ }^{*} P \leq 0.05 ;{ }^{* *} P \leq 0.01 ;{ }^{* * *} P$ $\leq 0.001$. All $P$ values were calculated using Tukey's Multiple Comparison Test. 


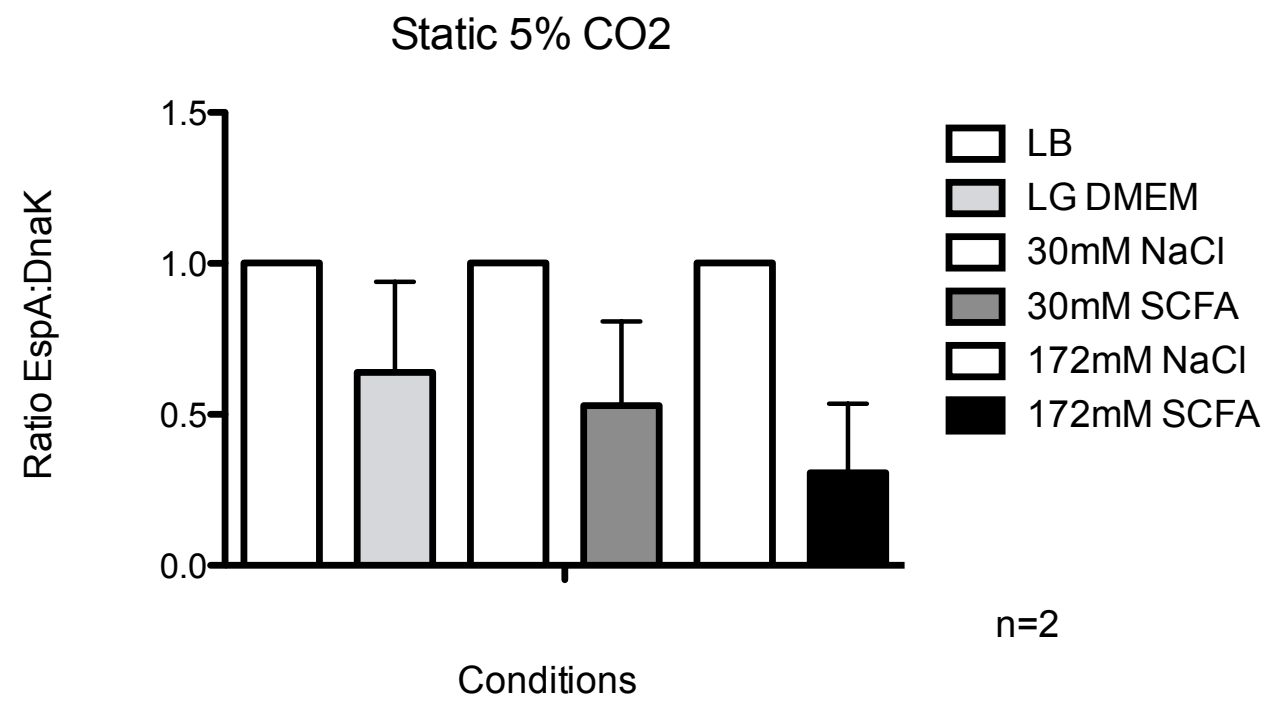

Figure 32. EspA Protein expression as observed under static condition with 5\% CO2

The data was generated by normalizing the experiments to the respective controls. In the case of LG DMEM, the control was LB, while in the case of the SCFA conditions, the control was the corresponding $\mathrm{NaCl}$ concentrations. This data is generated from 3 biological replicates. Data are presented as means \pm s.d.; $n=3 .{ }^{*} P \leq 0.05 ;{ }^{* *} P \leq 0.01 ;{ }^{* * *} P \leq 0.001$. All $P$ values were calculated using Tukey’s Multiple Comparison Test. 
Appendix D - Gene Analysis of T3SS Genes Using GFP Reporter Assay

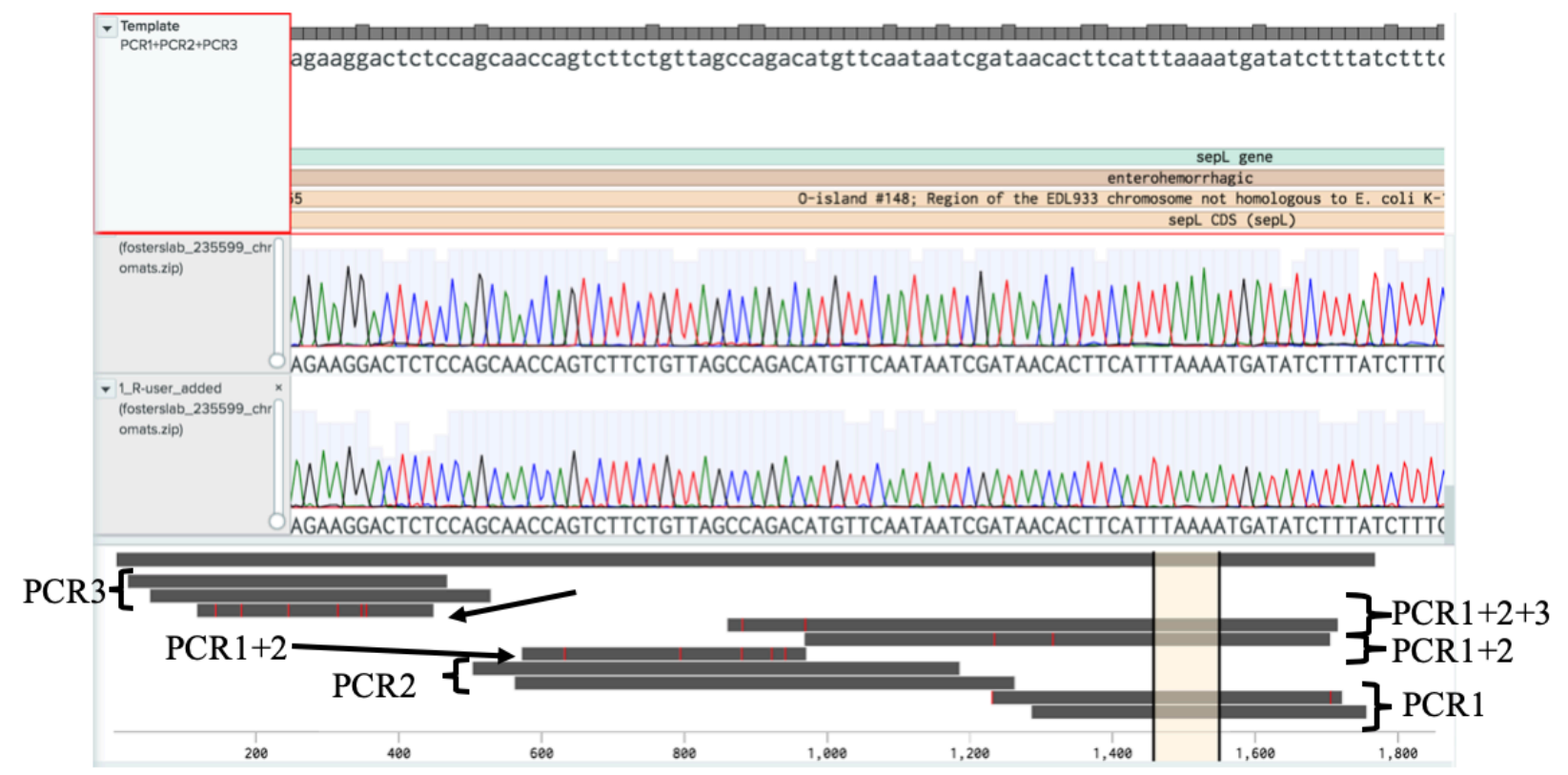

Figure 33. Sequence Data of the various PCR products to form the espA-GFP construct

The sequence data above shows that PCR1, PCR2 and PCR3 have the correct sequence. The sequence of PCR1+2 that were stitched together showed relatively good signal of having the correct sequence (see gray bars labelled PCR1+2). PCR1+2+3 however, did not have sufficient data in the center of the sequence to be sure that the sequence is correct. It is possible that resending the sequence in for sequencing will generate better sequence data. 


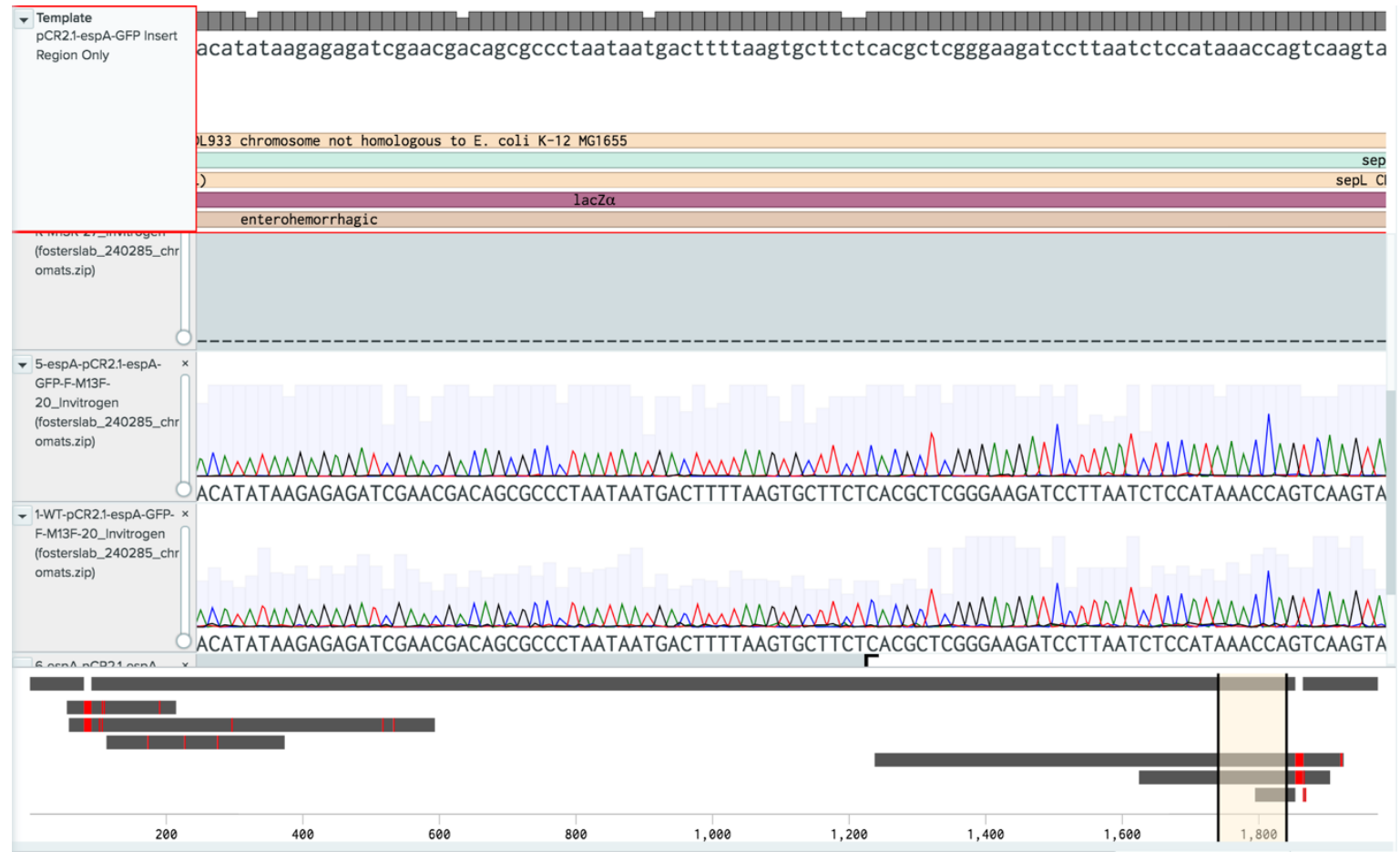

Figure 34. Sequencing data for 3 bacterial strains WT, and $\triangle \operatorname{espA}$ containing pCR2.1-espA-GFP

The gray bars at the bottom of the image represent successful nucleotide matches, while the red represent mismatches. This sequencing data, taken together with the plasmid digestion in figure 3, suggest that there was a successful insertion of the espA_GFP gene fusion into pCR2.1-TOPO. This allowed the commencement of the following step, which was transformation in the bacteria of interest followed by the GFP expression assay. 


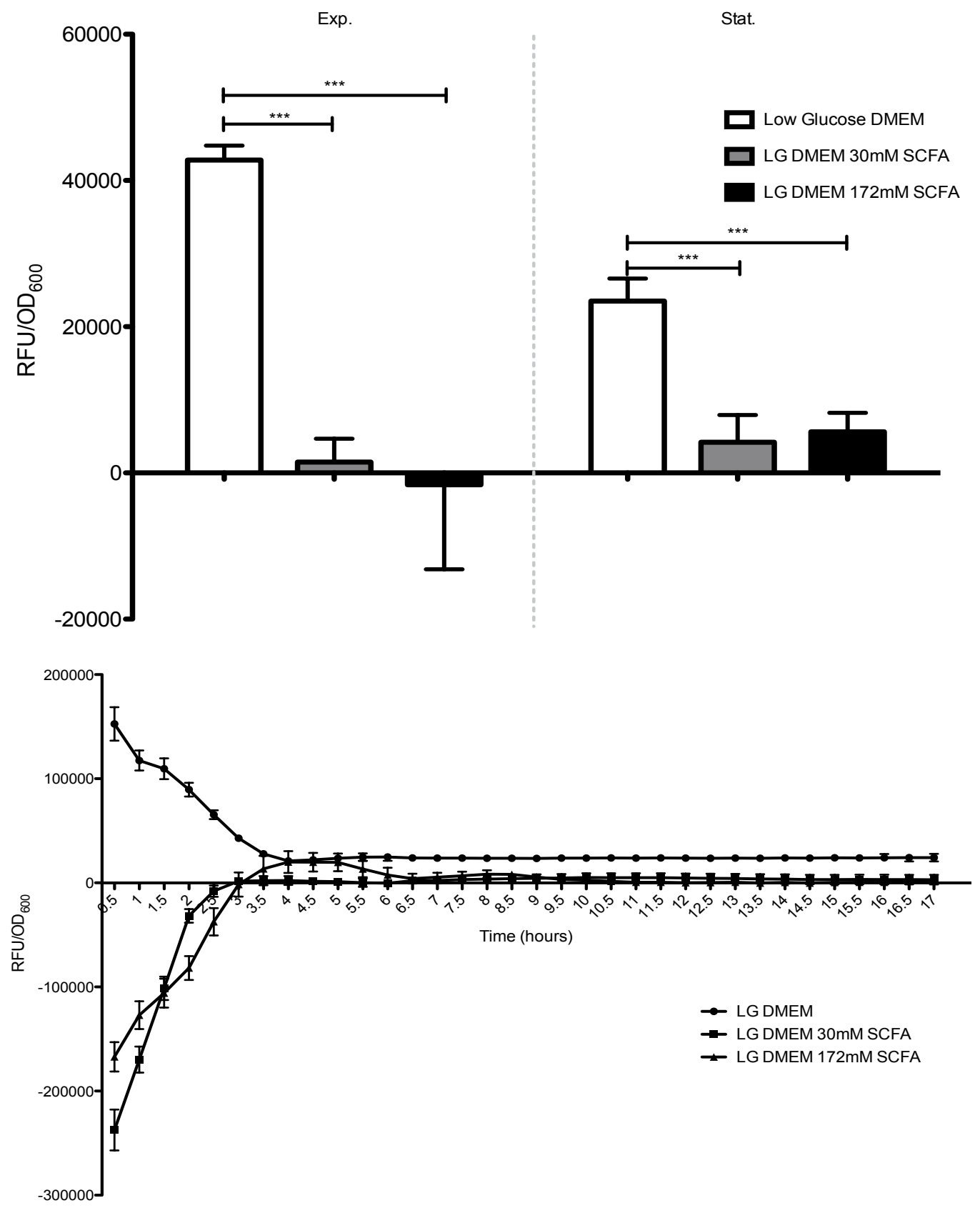

Figure 35. LG DMEM High Oxygen Control Subtracted

This data is the product of two experiments, one that contained the esp promoter prior to GFP, while the other, that contained an empty vector. The empty vector control was subtracted from the experimental values. This data is generated from 6 biological replicates. Data are presented as means \pm s.d.; $n=6 .{ }^{*} P \leq 0.05 ;{ }^{* *} P \leq 0.01 ;{ }^{* * *} P \leq 0.001$. All $P$ values were calculated using Tukey’s Multiple Comparison Test. 

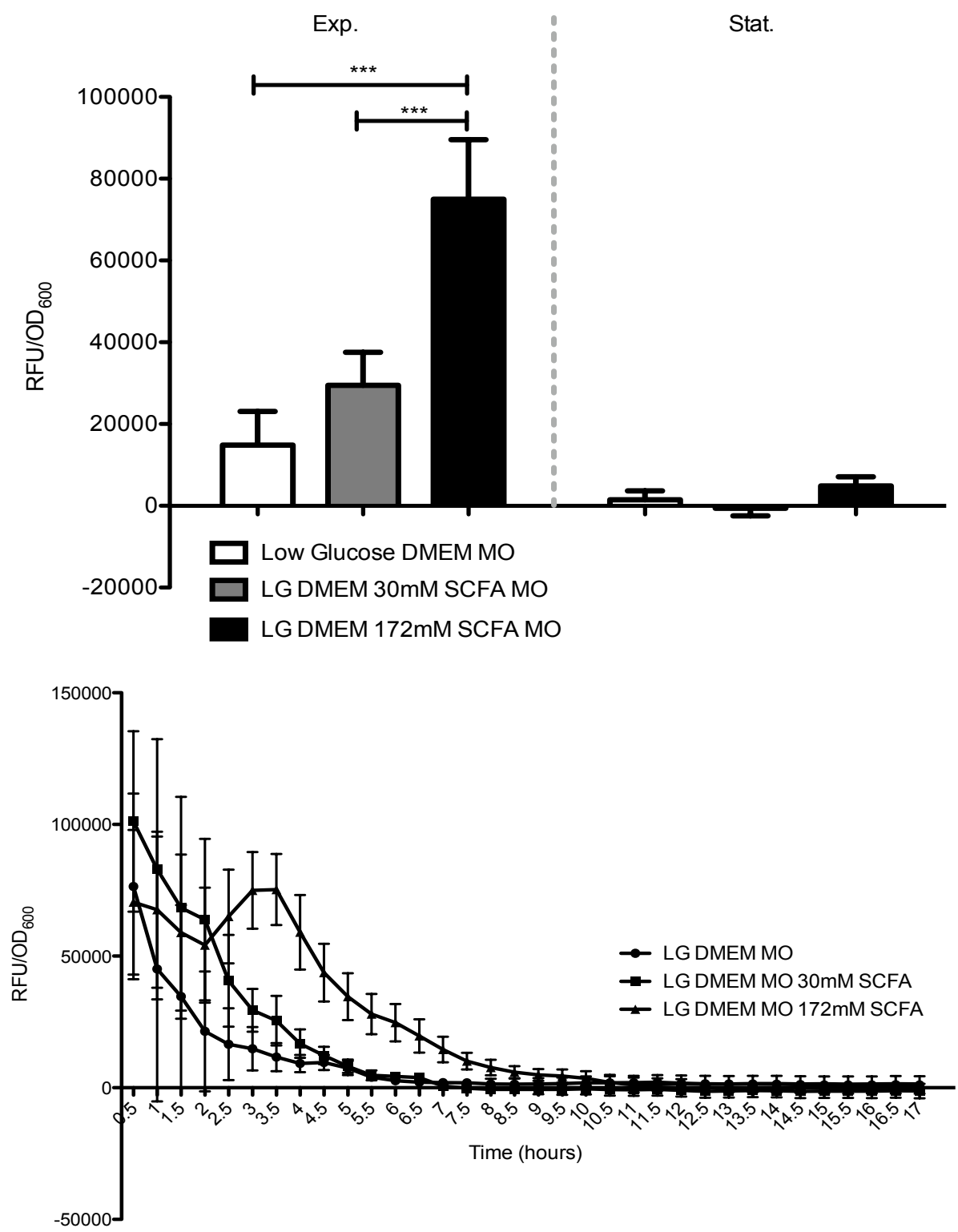

\section{Figure 36. LG DMEM Low Oxygen Control Subtracted}

This data is the product of two experiments, one that contained the esp promoter prior to GFP, while the other, that contained an empty vector. The empty vector control was subtracted from the experimental values. This data is generated from 6 biological replicates. Data are presented as means \pm s.d.; $n=6 .{ }^{*} P \leq 0.05 ;{ }^{* *} P \leq 0.01 ;{ }^{* * *} P \leq 0.001$. All $P$ values were calculated using Tukey's Multiple Comparison Test. 
Exp. Stat.
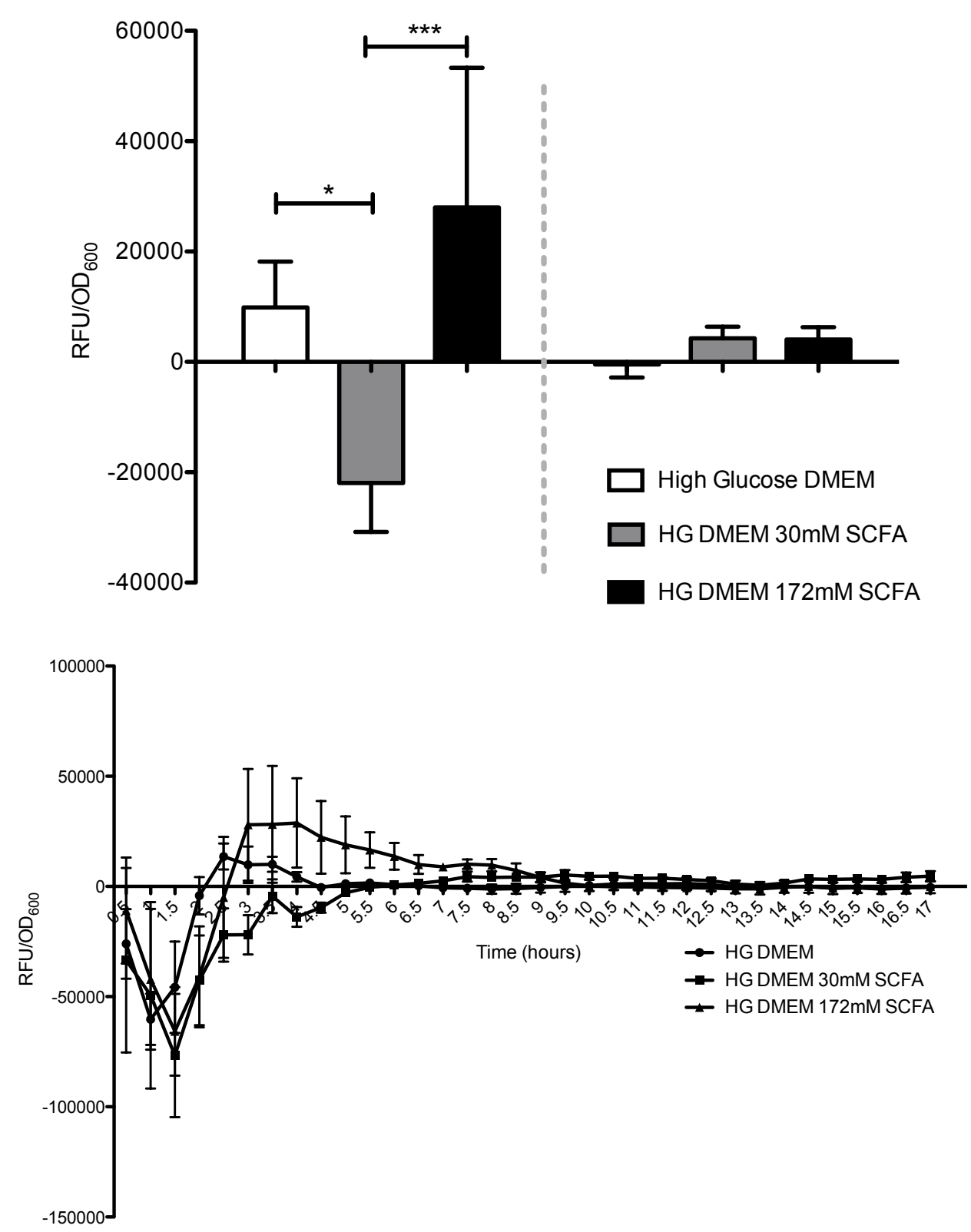

\section{Figure 37. HG DMEM High Oxygen Controls Subtracted}

This data is the product of two experiments, one that contained the esp promoter prior to GFP, while the other, that contained an empty vector. The empty vector control was subtracted from the experimental values. This data is generated from 6 biological replicates. Data are presented as means \pm s.d.; $n=6 .{ }^{*} P \leq 0.05 ;{ }^{* *} P \leq 0.01 ;{ }^{* * *} P \leq 0.001$. All $P$ values were calculated using Tukey's Multiple Comparison Test. 

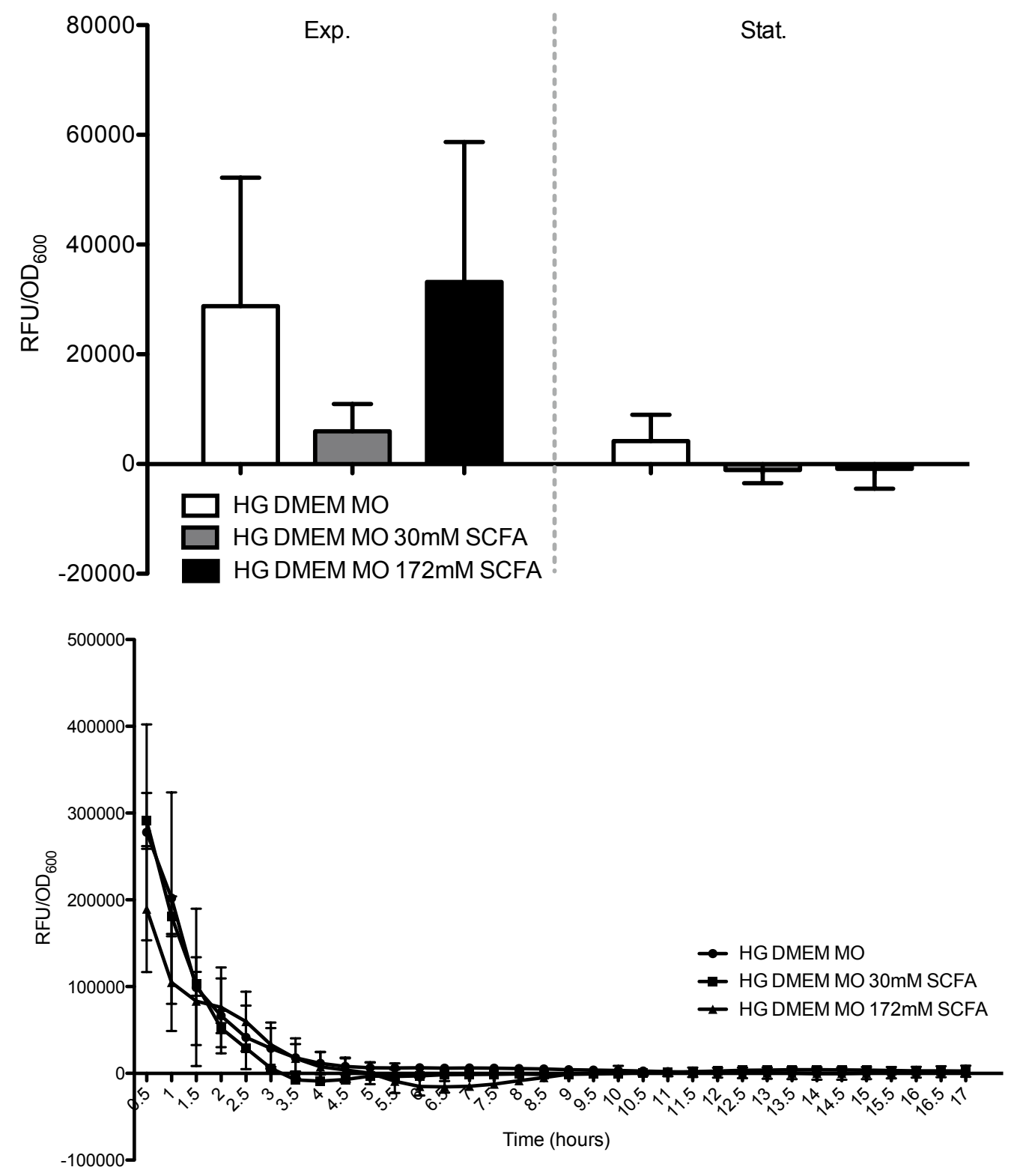

Figure 38. HG DMEM Low Oxygen Controls Subtracted

This data is the product of two experiments, one that contained the esp promoter prior to GFP, while the other, that contained an empty vector. The empty vector control was subtracted from the experimental values. This data is generated from 6 biological replicates. Data are presented as means \pm s.d.; $n=6 .{ }^{*} P \leq 0.05 ;{ }^{* *} P \leq 0.01 ;{ }^{* * *} P \leq 0.001$. All $P$ values were calculated using Tukey’s Multiple Comparison Test. 


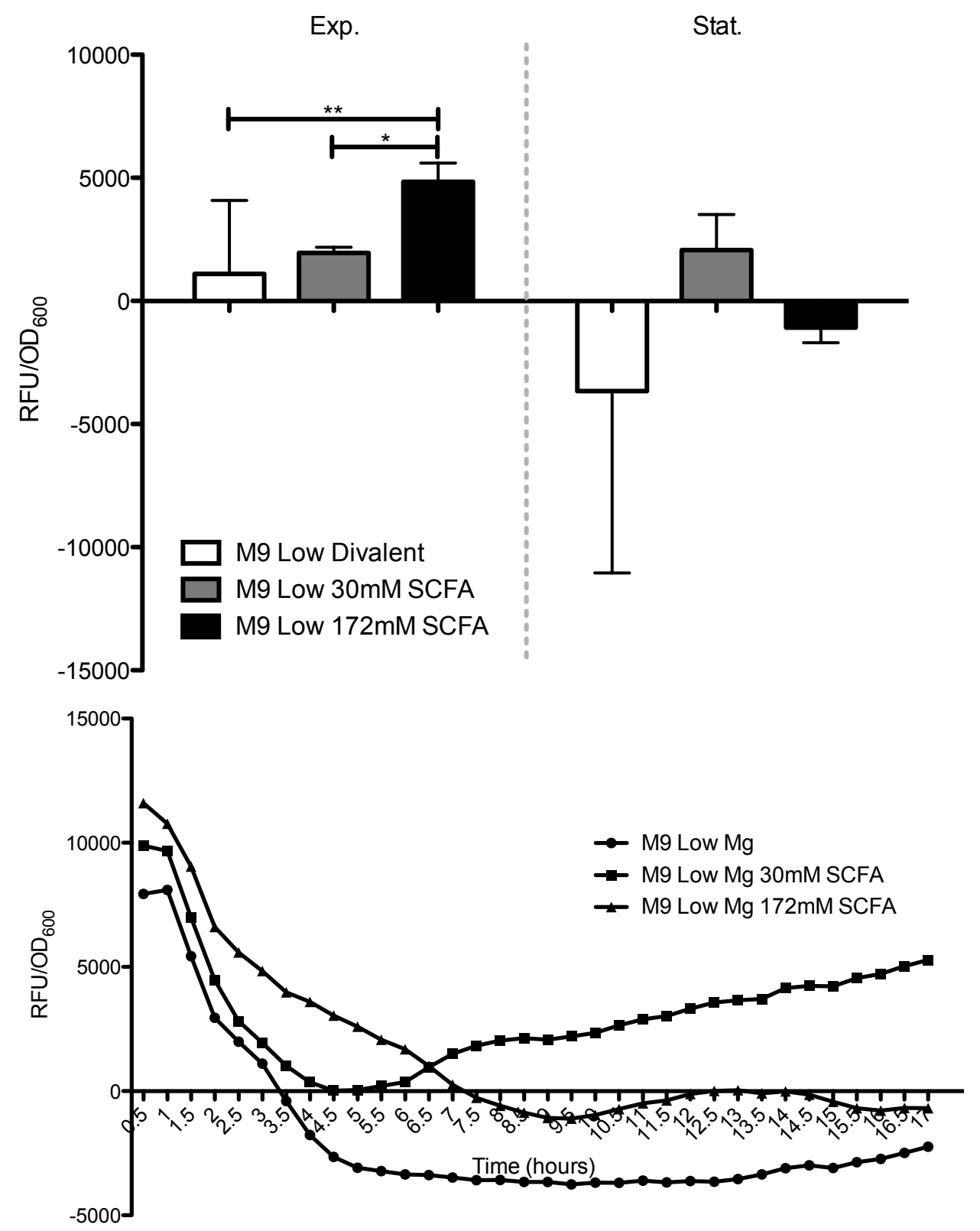

\section{Figure 39. M9 Low Magnesium Corrected}

This data is the product of two experiments, one that contained the esp promoter prior to GFP, while the other, that contained an empty vector. The empty vector control was subtracted from the experimental values. This data is generated from 6 biological replicates. Data are presented as means \pm s.d.; $n=6 .{ }^{*} P \leq 0.05 ;{ }^{* *} P \leq 0.01 ;{ }^{* * *} P \leq 0.001$. All $P$ values were calculated using Tukey’s Multiple Comparison Test. 

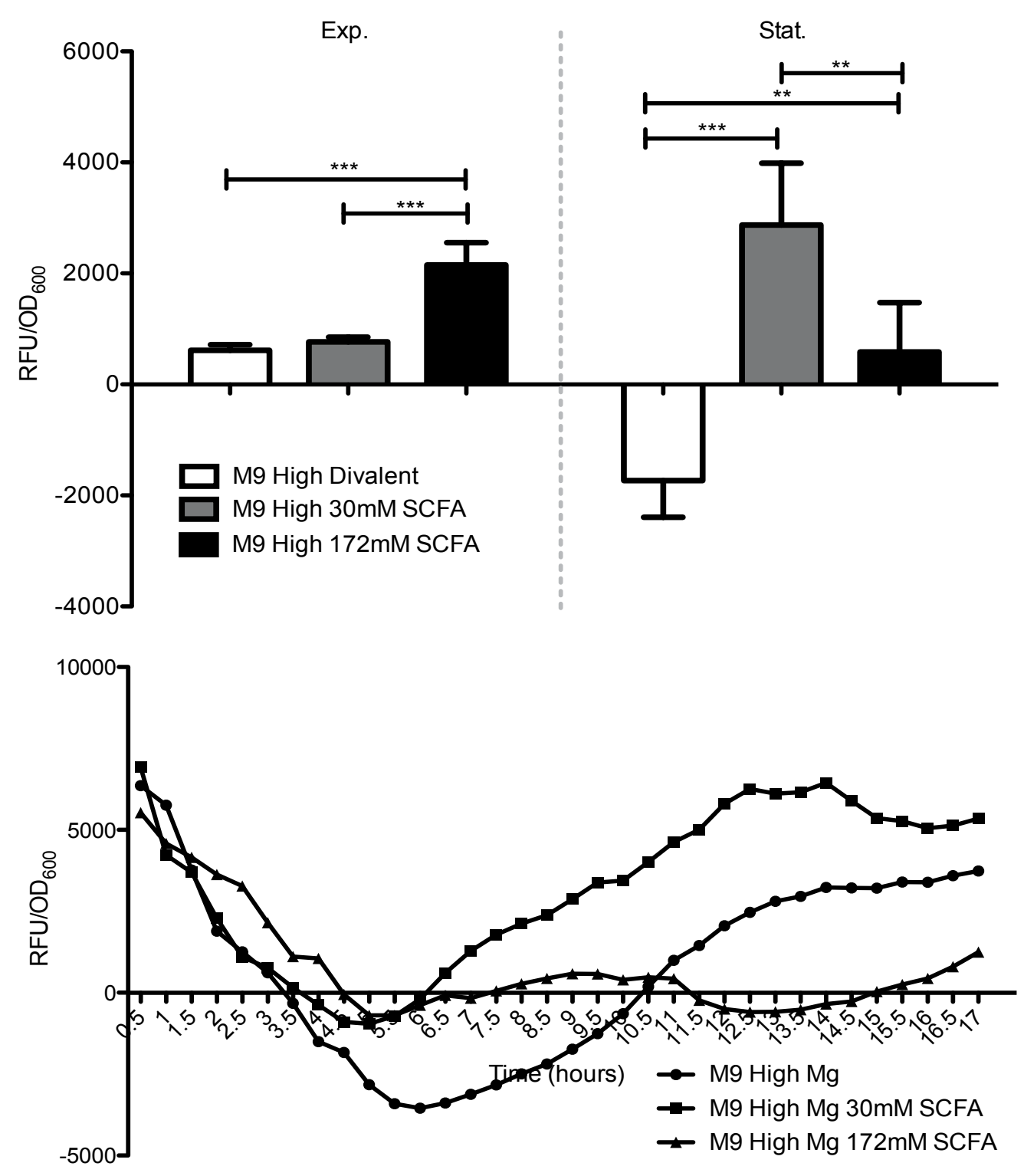

\section{Figure 40. M9 High Magnesium Corrected}

This data is the product of two experiments, one that contained the esp promoter prior to GFP, while the other, that contained an empty vector. The empty vector control was subtracted from the experimental values. This data is generated from 6 biological replicates. Data are presented as means \pm s.d.; $n=6 .{ }^{*} P \leq 0.05 ;{ }^{* *} P \leq 0.01 ;{ }^{* * *} P \leq 0.001$. All $P$ values were calculated using Tukey’s Multiple Comparison Test. 


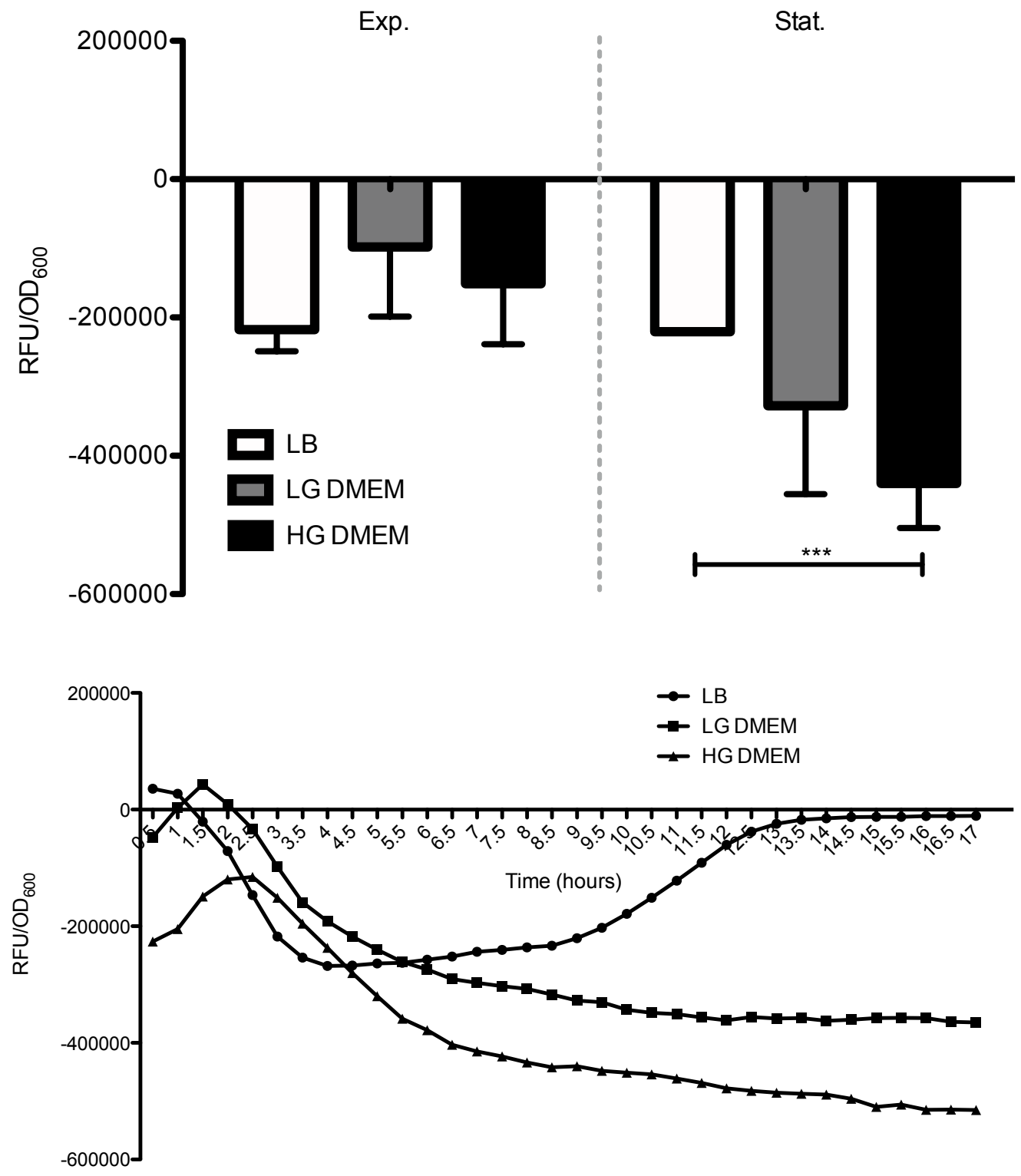

Figure 41. Reactive Oxygen Species Controls Subtracted

This data is the product of two experiments, one that contained the esp promoter prior to GFP, while the other, that contained an empty vector. The empty vector control was subtracted from the experimental values. This data is generated from 6 biological replicates. Data are presented as means \pm s.d.; $n=6 .{ }^{*} P \leq 0.05 ;{ }^{* *} P \leq 0.01 ;{ }^{* * *} P \leq 0.001$. All $P$ values were calculated using Tukey's Multiple Comparison Test. 


\section{REFERENCES}

1. $\quad$ Escherich, T. Die darmbakterien des neugeborenen und säuglings. Fortshr. Med. 3:5-15522, 547-554 (1885).

2. Riley, L. W. et al. Hemorrhagic Colitis Associated with a Rare Escherichia coli Serotype. N. Engl. J. Med. 308, 681-685 (1983).

3. Levine, M. M. Escherichia coli that Cause Diarrhea: Enterotoxigenic, Enteropathogenic, Enteroinvasive, Enterohemorrhagic, and Enteroadherent. J. Infect. Dis. 155, 377-389 (1987).

4. Kaper, J. B., Nataro, J. P. \& Mobley, H. L. T. Pathogenic Escherichia coli. Nat. Rev. Microbiol. 2, 123-140 (2004).

5. Mark Taylor, C. Enterohaemorrhagic Escherichia coli and Shigella dysenteriae type 1induced haemolytic uraemic syndrome. Pediatr. Nephrol. 23, 1425-31 (2008).

6. Nguyen, Y. \& Sperandio, V. Enterohemorrhagic E. coli (EHEC) pathogenesis. Front. Cell. Infect. Microbiol. 2, 90 (2012).

7. Menne, J. et al. Validation of treatment strategies for enterohaemorrhagic Escherichia coli O104:H4 induced haemolytic uraemic syndrome: case-control study. BMJ 345, e4565 (2012).

8. $\quad$ Lynch, M., Painter, J., Woodruff, R., Braden, C. \& Centers for Disease Control and Prevention. Surveillance for foodborne-disease outbreaks--United States, 1998-2002. MMWR. Surveill. Summ. 55, 1-42 (2006).

9. Scheiring, J., Andreoli, S. P. \& Zimmerhackl, L. B. Treatment and outcome of Shigatoxin-associated hemolytic uremic syndrome (HUS). Pediatr. Nephrol. 23, 1749-1760 (2008).

10. Sandvig, K. et al. Pathways followed by ricin and Shiga toxin into cells. Histochem. Cell Biol. 117, 131-141 (2002).

11. Karmali, M. A., Petric, M., Lim, C., Fleming, P. C. \& Steele, B. T. Escherichia coli Cytotoxin, Haemolytic-Uraemic Syndrome, And Haemorrhagic Colitis. Lancet 322, 1299-1300 (1983).

12. Boyce, T. G., Swerdlow, D. L. \& Griffin, P. M. Escherichia coli O157:H7 and the Hemolytic-Uremic Syndrome. N. Engl. J. Med. 333, 364-368 (1995).

13. Kimmitt, P. T., Harwood, C. R. \& Barer, M. R. Toxin gene expression by shiga toxinproducing Escherichia coli: the role of antibiotics and the bacterial SOS response. Emerg. Infect. Dis. 6, 458-65 (2000). 
14. Karmali, M., Petric, M., Steele, B. \& Lim, C. Sporadic Cases Of Haemolytic-Uraemic Syndrome Associated With Faecal Cytotoxin And Cytotoxin-Producing Escherichia coli In Stools. Lancet 321, 619-620 (1983).

15. O’Flaherty, E., Solimini, A. G., Pantanella, F., De Giusti, M. \& Cummins, E. Human exposure to antibiotic resistant-Escherichia coli through irrigated lettuce. Environ. Int. 122, 270-280 (2019).

16. Keene, W. E. et al. A Prolonged Outbreak of Escherichia coli O157:H7 Infections Caused by Commercially Distributed Raw Milk. J. Infect. Dis. 176, 815-818 (1997).

17. Bruce, M. G. et al. Lake-Associated Outbreak of Escherichia coli O157:H7 in Clark County, Washington, August 1999. Arch. Pediatr. Adolesc. Med. 157, 1016 (2003).

18. Health Agency of Canada, P. \& de santé publique du Canada, A. Canada Communicable Disease Report Relevé des maladies transmissibles au Canada An Outbreak of Escherichia coli O157:H7 Associated With a Children's Water Spray Park And Identified by Two Rounds of Pulsed-Field Gel Electrophoresis Testing.

19. Cambridge University Press, M. et al. Epidemiology and infection. Epidemiology \& Infection 122, (Cambridge University Press, 1987).

20. Keene, W. E. et al. A Swimming-Associated Outbreak of Hemorrhagic Colitis Caused by Escherichia coli O157:H7 and Shigella Sonnei. N. Engl. J. Med. 331, 579-584 (1994).

21. Hilborn", E. D. et al. An outbreak of Escherichia coli O157:H7 infections and haemolytic uraemic syndrome associated with consumption of unpasteurized apple cider. Epidemiol. Infect 124, (Cambridge University Press, 2019).

22. Keene, W. E. et al. An Outbreak of Escherichia coli O157:H7 Infections Traced to Jerky Made From Deer Meat. JAMA J. Am. Med. Assoc. 277, 1229 (1997).

23. Schuster, C. J. et al. Infectious disease outbreaks related to drinking water in Canada, 1974-2001. Can. J. Public Health 96, 254-8

24. Sinclair, J. F. et al. Cell surface-localized nucleolin is a eukaryotic receptor for the adhesin intimin-gamma of enterohemorrhagic Escherichia coli O157:H7. J. Biol. Chem. 277, 2876-85 (2002).

25. Cimolai, N., Morrison, B. J. \& Carter, J. E. Risk Factors for the Central Nervous System Manifestations of Gastroenteritis-Associated Hemolytic-Uremic Syndrome. Pediatrics 90, (1992).

26. Griffin, P. M. \& Tauxe, R. V. The Epidemiology of Infections Caused by Escherichia coli O157: H7, Other Enterohemorrhagic E. coli, and the Associated Hemolytic Uremic Syndrome. Epidemiol. Rev. 13, 60-98 (1991). 
27. Pickering, L.K., T.G. Obrig, and F. B. S. Hemolytic uremic syndrome and.1.pd. Pediatr Infect Dis J 13(6):, 459-75 (1994).

28. Siegler, R. L., Christofferson, R. D., Milligan, M. K. \& Pavia, A. T. A 20-Year Population-Based Study of Postdiarrheal Hemolytic Uremic Syndrome in Utah. Pediatrics 94, (1994).

29. Ngwenya, N. \& Chirwa, E. M. N. Biological removal of cationic fission products from nuclear wastewater. Water Sci. Technol. 63, 124-128 (2011).

30. Cimolai, N., Morrison, B. J. \& Carter, J. E. Risk Factors for the Central Nervous System Manifestations of Gastroenteritis-Associated Hemolytic-Uremic Syndrome. Pediatrics 90, (1992).

31. Corrigan, J. J. \& Boineau, F. G. Hemolytic-Uremic Syndrome.

32. March, S. B. \& Ratnam, S. Sorbitol-MacConkey medium for detection of Escherichia coli O157:H7 associated with hemorrhagic colitis. J. Clin. Microbiol. 23, 869-72 (1986).

33. Gould, L. H. Update: Recommendations for Diagnosis of Shiga Toxin-Producing Escherichia coli Infections by Clinical Laboratories. Clin. Microbiol. Newsl. 34, 75-83 (2012).

34. D'LIMA, C. B. \& SUSLOW, T. V. Comparative Evaluation of Practical Functionality of Rapid Test Format Kits for Detection of Escherichia coli O157:H7 on Lettuce and Leafy Greens. J. Food Prot. 72, 2461-2470 (2009).

35. Park, C. H., Gates, K. M., Vandel, N. M. \& Hixon, D. L. Isolation of Shiga-like toxin producing Escherichia coli (O157 and non-O157) in a community hospital. Diagn. Microbiol. Infect. Dis. 26, 69-72 (1996).

36. Lee, J. H. \& Stein, B. D. Antimicrobials effective for inhibition of enterohaemorrhagic Escherichia coli strains O26, O111, and O157 and their effects on Shiga toxin releases. $J$. Microbiol. Biotechnol. 19, 1238-43 (2009).

37. Carter, A. O. et al. A Severe Outbreak of Escherichia coli O157:H7-Associated Hemorrhagic Colitis in a Nursing Home. N. Engl. J. Med. 317, 1496-1500 (1987).

38. Tarr, P. et al. Hemolytic Uremic Syndrome Epidemiology: A Population-Based Study in King County, Washington, 1971 to 1980. Pediatrics 80, 41-45 (1997).

39. Plunkett, G., Rose, D. J., Durfee, T. J. \& Blattner, F. R. Sequence of Shiga toxin 2 phage 933W from Escherichia coli O157:H7: Shiga toxin as a phage late-gene product. $J$. Bacteriol. 181, 1767-78 (1999).

40. Neely, M. N. \& Friedman, D. I. Functional and genetic analysis of regulatory regions of 
coliphage H-19B: location of shiga-like toxin and lysis genes suggest a role for phage functions in toxin release. Mol. Microbiol. 28, 1255-67 (1998).

41. Kaper, J. B. \& O'Brien, A. D. Escherichia coli O157:H7 and other shiga toxin-producing E. coli strains. (ASM Press, 1998).

42. Bavaro, M. F. Escherichia coli O157: What every internist and gastroenterologist should know. Curr. Gastroenterol. Rep. 11, 301-306 (2009).

43. Siegler, R. L. The Hemolytic Uremic Syndrome. Pediatr. Clin. North Am. 42, 1505-1529 (1995).

44. Lim, J. Y., Yoon, J. \& Hovde, C. J. A brief overview of Escherichia coli O157:H7 and its plasmid O157. J. Microbiol. Biotechnol. 20, 5-14 (2010).

45. Mellies, J. L., Elliott, S. J., Sperandio, V., Donnenberg, M. S. \& Kaper, J. B. The Per regulon of enteropathogenic Escherichia coli: identification of a regulatory cascade and a novel transcriptional activator, the locus of enterocyte effacement (LEE)-encoded regulator (Ler). Mol. Microbiol. 33, 296-306 (1999).

46. Elliott, S. J. et al. The complete sequence of the locus of enterocyte effacement (LEE) from enteropathogenic Escherichia coli E2348/69. Mol. Microbiol. 28, 1-4 (2002).

47. Muller, D. et al. Comparative Analysis of the Locus of Enterocyte Effacement and Its Flanking Regions. Infect. Immun. 77, 3501-3513 (2009).

48. Castillo, A., Eguiarte, L. E. \& Souza, V. A genomic population genetics analysis of the pathogenic enterocyte effacement island in Escherichia coli: the search for the unit of selection. Proc. Natl. Acad. Sci. U. S. A. 102, 1542-7 (2005).

49. Jarvis, K. G. \& Kaper, J. B. Secretion of extracellular proteins by enterohemorrhagic Escherichia coli via a putative type III secretion system. Infect. Immun. 64, 4826-9 (1996).

50. Diepold, A. \& Wagner, S. Assembly of the bacterial type III secretion machinery. FEMS Microbiol. Rev. 38, 802-822 (2014).

51. Zemanick, E. T. et al. Reliability of Quantitative Real-Time PCR for Bacterial Detection in Cystic Fibrosis Airway Specimens. PLoS One 5, e15101 (2010).

52. Luo, Y. et al. Crystal structure of enteropathogenic Escherichia coli intimin-receptor complex. Nature 405, 1073-7 (2000).

53. Green, E. R. \& Mecsas, J. Bacterial Secretion Systems-An overview CHAPTER SUMMARY. doi:10.1128/microbiolspec.VMBF-0012-2015

54. Kresse, A. U., Rohde, M. \& Guzmán, C. A. The EspD protein of enterohemorrhagic 
Escherichia coli is required for the formation of bacterial surface appendages and is incorporated in the cytoplasmic membranes of target cells. Infect. Immun. 67, 4834-42 (1999).

55. Ide, T. et al. Characterization of translocation pores inserted into plasma membranes by type III-secreted Esp proteins of enteropathogenic Escherichia coli. Cell. Microbiol. 3, 669-679 (2001).

56. Crepin, V. F. et al. Structural and functional studies of the enteropathogenic Escherichia coli type III needle complex protein EscJ. Mol. Microbiol. 55, 1658-1670 (2005).

57. Gauthier, A., Puente, J. L. \& Finlay, B. B. Secretin of the enteropathogenic Escherichia coli type III secretion system requires components of the type III apparatus for assembly and localization. Infect. Immun. 71, 3310-9 (2003).

58. Demers, J.-P. et al. High-resolution structure of the Shigella type-III secretion needle by solid-state NMR and cryo-electron microscopy. Nat. Commun. 5, 4976 (2014).

59. Deng, W. et al. Dissecting virulence: systematic and functional analyses of a pathogenicity island. Proc. Natl. Acad. Sci. U. S. A. 101, 3597-602 (2004).

60. O'Connell, C. B. et al. SepL, a protein required for enteropathogenic Escherichia coli type III translocation, interacts with secretion component SepD. Mol. Microbiol. 52, 16131625 (2004).

61. Creasey, E. A., Delahay, R. M., Daniell, S. J. \& Frankel, G. Yeast two-hybrid system survey of interactions between LEE-encoded proteins of enteropathogenic Escherichia coli. Microbiology 149, 2093-2106 (2003).

62. Andrade, A., Pardo, J. P., Espinosa, N., Pérez-Hernández, G. \& González-Pedrajo, B. Enzymatic characterization of the enteropathogenic Escherichia coli type III secretion ATPase EscN. Arch. Biochem. Biophys. 468, 121-127 (2007).

63. Creasey, E. A. et al. CesT is a bivalent enteropathogenic Escherichia coli chaperone required for translocation of both Tir and Map. Mol. Microbiol. 47, 209-221 (2002).

64. Gauthier, A. \& Finlay, B. B. Translocated intimin receptor and its chaperone interact with ATPase of the type III secretion apparatus of enteropathogenic Escherichia coli. $J$. Bacteriol. 185, 6747-55 (2003).

65. Elliott, S. J. et al. A gene from the locus of enterocyte effacement that is required for enteropathogenic Escherichia coli to increase tight-junction permeability encodes a chaperone for EspF. Infect. Immun. 70, 2271-7 (2002).

66. Abe, A. et al. Enteropathogenic Escherichia coli translocated intimin receptor, Tir, requires a specific chaperone for stable secretion. Mol. Microbiol. 33, 1162-1175 (2002). 
67. Neves, B. C. et al. CesD2 of enteropathogenic Escherichia coli is a second chaperone for the type III secretion translocator protein EspD. Infect. Immun. 71, 2130-41 (2003).

68. Creasey, E. A. et al. CesAB is an enteropathogenic Escherichia coli chaperone for the type-III translocator proteins EspA and EspB. Microbiology 149, 3639-3647 (2003).

69. Wainwright, L. A. \& Kaper, J. B. EspB and EspD require a specific chaperone for proper secretion from enteropathogenic Escherichia coli. Mol. Microbiol. 27, 1247-1260 (1998).

70. O’Connell, C. B. et al. SepL, a protein required for enteropathogenic Escherichia coli type III translocation, interacts with secretion component SepD. Mol. Microbiol. 52, 16131625 (2004).

71. Hicks, S., Frankel, G., Kaper, J. B., Dougan, G. \& Phillips, A. D. Role of intimin and bundle-forming pili in enteropathogenic Escherichia coli adhesion to pediatric intestinal tissue in vitro. Infect. Immun. 66, 1570-8 (1998).

72. Donnenberg, M. S. et al. Role of the eaeA Gene in Experimental Enteropathogenic Escherichia coil Infection Key words: viru-lence genes * microbial pathogenesis-diarrhea * immune re-sponse * fever. J. Clin. Invest 92, (1993).

73. Donnenberg, M. S. et al. The Role of the eae Gene of Enterohemorrhagic Escherichia coli in Intimate Attachment In Vitro and in a Porcine Model Key words: bacterial adhesion* disease models * genetics * microbial * mutations * pathogenicity Introduction. J. Clin. Invest 92, (1993).

74. Cleary, J. et al. Enteropathogenic Escherichia coli (EPEC) adhesion to intestinal epithelial cells: role of bundle-forming pili (BFP), EspA filaments and intimin. Microbiology 150, 527-538 (2004).

75. Rosenshine, I., Donnenberg, M. S., Kaper, J. B. \& Finlay, B. B. Signal transduction between enteropathogenic Escherichia coli (EPEC) and epithelial cells: EPEC induces tyrosine phosphorylation of host cell proteins to initiate cytoskeletal rearrangement and bacterial uptake. EMBO J. 11, 3551-3560 (1992).

76. Kenny, B. et al. Enteropathogenic E. coli (EPEC) Transfers Its Receptor for Intimate Adherence into Mammalian Cells. Cell 91, 511-520 (1997).

77. DeVinney, R., Puente, J. L., Gauthier, A., Goosney, D. \& Finlay, B. B. Enterohaemorrhagic and enteropathogenic Escherichia coli use a different Tir-based mechanism for pedestal formation. Mol. Microbiol. 41, 1445-1458 (2001).

78. Kenny, B. The enterohaemorrhagic Escherichia coli (serotype O157:H7) Tir molecule is not functionally interchangeable for its enteropathogenic E. coli (serotype O127:H6) homologue. Cell. Microbiol. 3, 499-510 (2001). 
79. Campellone, K. G., Giese, N., Tipper, O. J. \& Leong, J. M. A tyrosine-phosphorylated 12amino-acid sequence of enteropathogenic Escherichia coli Tir binds the host adaptor protein Nck and is required for Nck localization to actin pedestals. Mol. Microbiol. 43, 1227-1241 (2002).

80. Schüller, S. et al. Tir phosphorylation and Nck/N-WASP recruitment by enteropathogenic and enterohaemorrhagic Escherichia coli during ex vivo colonization of human intestinal mucosa is different to cell culture models. Cell. Microbiol. 9, 1352-1364 (2007).

81. Millard, T. H., Dawson, J. \& Machesky, L. M. Characterisation of IRTKS, a novel IRSp53/MIM family actin regulator with distinct filament bundling properties. J. Cell Sci. 120, 1663-72 (2007).

82. Vingadassalom, D. et al. Insulin receptor tyrosine kinase substrate links the $E$. coli O157:H7 actin assembly effectors Tir and $\operatorname{EspF}(\mathrm{U})$ during pedestal formation. Proc. Natl. Acad. Sci. U. S. A. 106, 6754-9 (2009).

83. Garmendia, J. et al. TccP is an enterohaemorrhagic Escherichia coli O157:H7 type III effector protein that couples Tir to the actin-cytoskeleton+. Cell. Microbiol. 6, 1167-1183 (2004).

84. Mousnier, A. et al. Cortactin recruitment by enterohemorrhagic Escherichia coli O157:H7 during infection in vitro and ex vivo. Infect. Immun. 76, 4669-76 (2008).

85. Cantarelli, V. V. et al. Tyrosine phosphorylation controls cortactin binding to two enterohaemorrhagic Escherichia coli effectors: Tir and EspFu/TccP. Cell. Microbiol. 9, 1782-1795 (2007).

86. McNamara, B. P. et al. Translocated EspF protein from enteropathogenic Escherichia coli disrupts host intestinal barrier function. J. Clin. Invest. 107, 621-9 (2001).

87. Nougayrede, J.-P. \& Donnenberg, M. S. Enteropathogenic Escherichia coli EspF is targeted to mitochondria and is required to initiate the mitochondrial death pathway. Cell. Microbiol. 6, 1097-1111 (2004).

88. Kenny, B. \& Jepson, M. Targeting of an enteropathogenic Escherichia coli (EPEC) effector protein to host mitochondria. Cell. Microbiol. 2, 579-590 (2000).

89. Kenny, B. et al. Co-ordinate regulation of distinct host cell signalling pathways by multifunctional enteropathogenic Escherichia coli effector molecules. Mol. Microbiol. 44, 1095-1107 (2002).

90. Hodges, K., Alto, N. M., Ramaswamy, K., Dudeja, P. K. \& Hecht, G. The enteropathogenic Escherichia coli effector protein EspF decreases sodium hydrogen exchanger 3 activity. Cell. Microbiol. 10, 1735-1745 (2008). 
91. Kanack, K. J., Crawford, J. A., Tatsuno, I., Karmali, M. A. \& Kaper, J. B. SepZ/EspZ is secreted and translocated into HeLa cells by the enteropathogenic Escherichia coli type III secretion system. Infect. Immun. 73, 4327-37 (2005).

92. Burland, V. et al. The complete DNA sequence and analysis of the large virulence plasmid of Escherichia coli O157:H7. Nucleic Acids Res. 26, 4196-4204 (1998).

93. Lim, J. Y., Sheng, H., Seo, K. S., Park, Y. H. \& Hovde, C. J. Characterization of an Escherichia coli $\mathrm{O} 157: \mathrm{H} 7$ plasmid $\mathrm{O} 157$ deletion mutant and its survival and persistence in cattle. Appl. Environ. Microbiol. 73, 2037-47 (2007).

94. Dziva, F. et al. EspP, a Type V-secreted serine protease of enterohaemorrhagic Escherichia coli O157:H7, influences intestinal colonization of calves and adherence to bovine primary intestinal epithelial cells. FEMS Microbiol. Lett. 271, 258-264 (2007).

95. Toth, I. et al. Influence of the 60-megadalton plasmid on adherence of Escherichia coli O157:H7 and genetic derivatives. Infect. Immun. 58, 1223-31 (1990).

96. Lim, J. Y., La, H. J., Sheng, H., Forney, L. J. \& Hovde, C. J. Influence of plasmid pO157 on Escherichia coli O157:H7 Sakai biofilm formation. Appl. Environ. Microbiol. 76, 963 6 (2010).

97. Tarr, P. I., Gordon, C. A. \& Chandler, W. L. Shiga-toxin-producing Escherichia coli and haemolytic uraemic syndrome. Lancet 365, 1073-1086 (2005).

98. Ho, T. D., Davis, B. M., Ritchie, J. M. \& Waldor, M. K. Type 2 secretion promotes enterohemorrhagic Escherichia coli adherence and intestinal colonization. Infect. Immun. 76, 1858-65 (2008).

99. Yoon, J. W. \& Hovde, C. J. All blood, No stool: enterohemorrhagic Escherichia coli O157:H7 infection. J. Vet. Sci. 9, 219 (2008).

100. Szabady, R. L., Lokuta, M. A., Walters, K. B., Huttenlocher, A. \& Welch, R. A. Modulation of Neutrophil Function by a Secreted Mucinase of Escherichia coli O157:H7. PLoS Pathog. 5, e1000320 (2009).

101. Richardson, S. E. et al. Experimental verocytotoxemia in rabbits. Infect. Immun. 60, 4154-67 (1992).

102. Karmali, M. A. Infection by verocytotoxin-producing Escherichia coli. Clin. Microbiol. Rev. 2, 15-38 (1989).

103. Strockbine, N. A. et al. Two toxin-converting phages from Escherichia coli O157:H7 strain 933 encode antigenically distinct toxins with similar biologic activities. Infect. Immun. 53, 135-40 (1986). 
104. Jackson, M. P., Neill, R. J., O’Brien, A. D., Holmes, R. K. \& Newland, J. W. Nucleotide sequence analysis and comparison of the structural genes for Shiga-like toxin I and Shigalike toxin II encoded by bacteriophages from Escherichia coli 933. FEMS Microbiol. Lett. 44, 109-114 (1987).

105. Strockbine, N. A., Marques, L. R., Holmes, R. K. \& O'Brien, A. D. Characterization of monoclonal antibodies against Shiga-like toxin from Escherichia coli. Infect. Immun. 50, 695-700 (1985).

106. Head, S. C., Karmalisg, M. A. \& Lingwood, C. A. Preparation of VT1 and VT2 Hybrid Toxins from Their Purified Dissociated Subunits EVIDENCE FOR B SUBUNIT MODULATION OF A SUBUNIT FUNCTION*. THE JOURNAL OF BIOLOGICAL CHEMISTRY 266, (1991).

107. Louise, C. B. \& Obrig, T. G. Specific Interaction of Escherichia coli 0157:H7-Derived Shiga-like Toxin II with Human Renal Endothelial Cells. J. Infect. Dis. 172, 1397-1401 (1995).

108. Ostroff, S. M. et al. Toxin Genotypes and Plasmid Profiles as Determinants of Systemic Sequelae in Escherichia coli O157:H7 Infections. J. Infect. Dis. 160, 994-998 (1989).

109. Plunkett, G., Rose, D. J., Durfee, T. J. \& Blattner, F. R. Sequence of Shiga toxin 2 phage 933W from Escherichia coli O157:H7: Shiga toxin as a phage late-gene product. $J$. Bacteriol. 181, 1767-78 (1999).

110. Neely, M. N. \& Friedman, D. I. Functional and genetic analysis of regulatory regions of coliphage H-19B: location of shiga-like toxin and lysis genes suggest a role for phage functions in toxin release. Mol. Microbiol. 28, 1255-1267 (1998).

111. PTASHNE, M. Specific Binding of the $\lambda$ Phage Repressor to $\lambda$ DNA. Nature 214, 232 234 (1967).

112. Butala, M., Žgur-Bertok, D. \& Busby, S. J. W. The bacterial LexA transcriptional repressor. Cell. Mol. Life Sci. 66, 82-93 (2009).

113. Little, J. W., Edmiston, S. H., Pacelli, L. Z. \& Mount, D. W. Cleavage of the Escherichia coli lexA protein by the recA protease. Proc. Natl. Acad. Sci. U. S. A. 77, 3225-9 (1980).

114. Tyler, J. S., Mills, M. J. \& Friedman, D. I. The operator and early promoter region of the Shiga toxin type 2 -encoding bacteriophage $933 \mathrm{~W}$ and control of toxin expression. $J$. Bacteriol. 186, 7670-9 (2004).

115. Safdar, N., Said, A., Gangnon, R. E. \& Maki, D. G. Risk of Hemolytic Uremic Syndrome After Antibiotic Treatment of Escherichia coli O157:H7 Enteritis. JAMA 288, 996 (2002).

116. Reid, S. D., Herbelin, C. J., Bumbaugh, A. C., Selander, R. K. \& Whittam, T. S. Parallel 
evolution of virulence in pathogenic Escherichia coli. Nature 406, 64-67 (2000).

117. Hayashi, T. et al. Complete Genome Sequence of Enterohemorrhagic Escherichia coli O157:H7 and Genomic Comparison with a Laboratory Strain K-12. DNA Res. 8, 11-22 (2001).

118. Perna, N. T. et al. Genome sequence of enterohaemorrhagic Escherichia coli O157:H7. Nature 409, 529-533 (2001).

119. Leopold, S. R. et al. A precise reconstruction of the emergence and constrained radiations of Escherichia coli $\mathrm{O} 157$ portrayed by backbone concatenomic analysis. Proc. Natl. Acad. Sci. U. S. A. 106, 8713-8 (2009).

120. Jerse, A. E., Yu, J., Tall, B. D. \& Kaper, J. B. A genetic locus of enteropathogenic Escherichia coli necessary for the production of attaching and effacing lesions on tissue culture cells. Proc. Natl. Acad. Sci. U. S. A. 87, 7839-43 (1990).

121. McDaniel, T. K. \& Kaper, J. B. A cloned pathogenicity island from enteropathogenic Escherichia coli confers the attaching and effacing phenotype on E. coli K-12. Mol. Microbiol. 23, 399-407 (1997).

122. Barnett Foster, D. Modulation of the enterohemorrhagic E. coli virulence program through the human gastrointestinal tract. Virulence 4, 315-323 (2013).

123. Abe, H., Tatsuno, I., Tobe, T., Okutani, A. \& Sasakawa, C. Bicarbonate Ion Stimulates the Expression of Locus of Enterocyte Effacement-Encoded Genes in Enterohemorrhagic Escherichia coli O157:H7. Infect. Immun. 70, 3500-3509 (2002).

124. House, B. et al. Acid-stress-induced changes in enterohaemorrhagic Escherichia coli O157 : H7 virulence. Microbiology 155, 2907-2918 (2009).

125. Foster, J. W. Escherichia coli acid resistance: tales of an amateur acidophile. Nat. Rev. Microbiol. 2, 898-907 (2004).

126. Kus, J. V et al. Bile salts induce resistance to polymyxin in enterohemorrhagic Escherichia coli O157:H7. J. Bacteriol. 193, 4509-15 (2011).

127. Wibbenmeyer, J. A., Provenzano, D., Landry, C. F., Klose, K. E. \& Delcour, A. H. Vibrio cholerae $\mathrm{OmpU}$ and $\mathrm{OmpT}$ porins are differentially affected by bile. Infect. Immun. 70, $121-6$ (2002).

128. Garsin, D. A. Ethanolamine: a signal to commence a host-associated lifestyle? MBio 3, e00172-12 (2012).

129. Tobe, T., Nakanishi, N. \& Sugimoto, N. Activation of Motility by Sensing Short-Chain Fatty Acids via Two Steps in a Flagellar Gene Regulatory Cascade in Enterohemorrhagic 
Escherichia coli $\uparrow$. Infect. Immun. 79, 1016-1024 (2011).

130. Lackraj, T., Kim, J. I., Tran, S. \& Barnett Foster, D. E. Differential modulation of flagella expression in enterohaemorrhagic Escherichia coli O157: H7 by intestinal short-chain fatty acid mixes. Microbiology 162, 1761-1772 (2016).

131. Nakanishi, N. et al. Regulation of virulence by butyrate sensing in enterohaemorrhagic Escherichia coli. Microbiology 155, 521-530 (2009).

132. Herold, S., Paton, J. C., Srimanote, P. \& Paton, A. W. Differential effects of short-chain fatty acids and iron on expression of iha in Shiga-toxigenic Escherichia coli.

Microbiology 155, 3554-3563 (2009).

133. Tobe, T., Nakanishi, N. \& Sugimoto, N. Activation of motility by sensing short-chain fatty acids via two steps in a flagellar gene regulatory cascade in enterohemorrhagic Escherichia coli. Infect. Immun. 79, 1016-24 (2011).

134. Macfarlane, G. T., Gibson, G. R. \& Cummings, J. H. Comparison of fermentation reactions in different regions of the human colon. J. Appl. Bacteriol. 72, 57-64 (1992).

135. Marteyn, B. et al. Modulation of Shigella virulence in response to available oxygen in vivo. Nature 465, 355-358 (2010).

136. Alexeeva, S., Hellingwerf, K. J. \& Teixeira de Mattos, M. J. Requirement of ArcA for redox regulation in Escherichia coli under microaerobic but not anaerobic or aerobic conditions. J. Bacteriol. 185, 204-9 (2003).

137. Dibden, D. P. \& Green, J. In vivo cycling of the Escherichia coli transcription factor FNR between active and inactive states. Microbiology 151, 4063-4070 (2005).

138. Cotter, P. A., Melville, S. B., Albrecht, J. A. \& Gunsalus, R. P. Aerobic regulation of cytochrome $d$ oxidase ( $c y d A B \quad$ ) operon expression in Escherichia coli : roles of Fnr and ArcA in repression and activation. Mol. Microbiol. 25, 605-615 (1997).

139. Mellies, J. L., Barron, A. M. S. \& Carmona, A. M. Enteropathogenic and enterohemorrhagic Escherichia coli virulence gene regulation. Infect. Immun. 75, 4199210 (2007).

140. Kenny, B. et al. Enteropathogenic E. coli (EPEC) Transfers Its Receptor for Intimate Adherence into Mammalian Cells. Cell 91, 511-520 (1997).

141. de Jesus, M. C., Urban, A. A., Marasigan, M. E. \& Barnett Foster, D. E. Acid and BileSalt Stress of Enteropathogenic Escherichia coli Enhances Adhesion to Epithelial Cells and Alters Glycolipid Receptor Binding Specificity. J. Infect. Dis. 192, 1430-1440 (2005). 
142. Lackraj, T., Kim, J. I., Tran, S. \& Barnett Foster, D. E. Differential modulation of flagella expression in enterohaemorrhagic Escherichia coli O157: H7 by intestinal short-chain fatty acid mixes. Microbiology 162, 1761-1772 (2016).

143. Barnett Foster, D. Modulation of the enterohemorrhagic E. coli virulence program through the human gastrointestinal tract. Virulence 4, 315-323 (2013).

144. House, B. et al. Acid-stress-induced changes in enterohaemorrhagic Escherichia coli O157 : H7 virulence. Microbiology 155, 2907-2918 (2009).

145. Kus, J. V. et al. Bile Salts Induce Resistance to Polymyxin in Enterohemorrhagic Escherichia coli O157:H7. J. Bacteriol. 193, 4509-4515 (2011).

146. Beckett, D. Biotin Sensing at the Molecular Level. J. Nutr. 139, 167-170 (2009).

147. Said, H. M. Cell and Molecular Aspects of Human Intestinal Biotin Absorption. J. Nutr. 139, 158-162 (2009).

148. Beckett, D. Biotin Sensing: Universal Influence of Biotin Status on Transcription. Annu. Rev. Genet. 41, 443-464 (2007).

149. Otsuka, A. \& Abelson, J. The regulatory region of the biotin operon in Escherichia coli. Nature 276, 689-694 (1978).

150. Yang, B., Feng, L., Wang, F. \& Wang, L. Enterohemorrhagic Escherichia coli senses low biotin status in the large intestine for colonization and infection. Nat. Commun. 6, 6592 (2015).

151. Su, M. S.-W., Kao, H.-C., Lin, C.-N. \& Syu, W.-J. Gene 10017 encodes a second chaperone for EspA of enterohaemorrhagic Escherichia coli O157 : H7. Microbiology 154, 1094-1103 (2008).

152. Guttman, J. A. et al. Attaching and effacing pathogen-induced tight junction disruption in vivo. Cell. Microbiol. 8, 634-645 (2006).

153. Neves, B. C. et al. CesD2 of enteropathogenic Escherichia coli is a second chaperone for the type III secretion translocator protein EspD. Infect. Immun. 71, 2130-41 (2003).

154. Shaw, R. K., Daniell, S., Ebel, F., Frankel, G. \& Knutton, S. EspA filament-mediated protein translocation into red blood cells. Cell. Microbiol. 3, 213-222 (2001).

155. Jerse, A. E. et al. A genetic locus of enteropathogenic Escherichia coli necessary for the production of attaching and effacing lesions on tissue culture cells. Proc. Natl. Acad. Sci. 87, 7839-7843 (1990).

156. Knutton, S. et al. A novel EspA-associated surface organelle of enteropathogenic Escherichia coli involved in protein translocation into epithelial cells. EMBO J. 17, 2166- 
76 (1998).

157. Wilson, R. K., Shaw, R. K., Daniell, S., Knutton, S. \& Frankel, G. Role of EscF, a putative needle complex protein, in the type III protein translocation system of enteropathogenic Escherichia coli. Cell. Microbiol. 3, 753-762 (2001).

158. Daniell, S. J. et al. 3D structure of EspA filaments from enteropathogenic Escherichia coli. Mol. Microbiol. 49, 301-308 (2003).

159. Daniell, S. J. et al. The filamentous type III secretion translocon of enteropathogenic Escherichia coli. Cell. Microbiol. 3, 865-871 (2001).

160. Kresse, A. U., Rohde, M. \& Guzmán, C. A. The EspD protein of enterohemorrhagic Escherichia coli is required for the formation of bacterial surface appendages and is incorporated in the cytoplasmic membranes of target cells. Infect. Immun. 67, 4834-42 (1999).

161. Luo, W. \& Donnenberg, M. S. Analysis of the function of enteropathogenic Escherichia coli EspB by random mutagenesis. Infect. Immun. 74, 810-20 (2006).

162. Iizumi, Y. et al. The Enteropathogenic E. coli Effector EspB Facilitates Microvillus Effacing and Antiphagocytosis by Inhibiting Myosin Function. Cell Host Microbe 2, 383392 (2007).

163. Ide, T. et al. Characterization of translocation pores inserted into plasma membranes by type III-secreted Esp proteins of enteropathogenic Escherichia coli. Cell. Microbiol. 3, 669-679 (2001).

164. Deng, W. et al. Regulation of type III secretion hierarchy of translocators and effectors in attaching and effacing bacterial pathogens. Infect. Immun. 73, 2135-46 (2005).

165. Kresse, A. U., Beltrametti, F., Müller, A., Ebel, F. \& Guzmán, C. A. Characterization of SepL of enterohemorrhagic Escherichia coli. J. Bacteriol. 182, 6490-8 (2000).

166. Wang, D., Roe, A. J., McAteer, S., Shipston, M. J. \& Gally, D. L. Hierarchal type III secretion of translocators and effectors from Escherichia coli $\mathrm{O} 157: \mathrm{H} 7$ requires the carboxy terminus of SepL that binds to Tir. Mol. Microbiol. 69, 1499-1512 (2008).

167. Beltrametti, F., Kresse, A. U. \& Guzmán, C. A. Transcriptional regulation of the esp genes of enterohemorrhagic Escherichia coli. J. Bacteriol. 181, 3409-18 (1999).

168. Sharma, V. K. \& Zuerner, R. L. Role of hha and ler in transcriptional regulation of the esp operon of enterohemorrhagic Escherichia coli O157:H7. J. Bacteriol. 186, 7290-301 (2004).

169. Roe, A. J., Hoey, D. E. E. \& Gally, D. L. Regulation, secretion and activity of type III- 
secreted proteins of enterohaemorrhagic Escherichia coli O157. Biochem. Soc. Trans. 31, 98-103 (2003).

170. Lodato, P. B. \& Kaper, J. B. Post-transcriptional processing of the LEE4 operon in enterohaemorrhagic Escherichia coli. Mol. Microbiol. 71, 273-290 (2009).

171. Lackraj, T., Kim, J. I., Tran, S. \& Foster, D. E. B. Differential modulation of flagella expression in enterohaemorrhagic Escherichia coli O157: H7 by intestinal short-chain fatty acid mixes. Microbiology 162, 1761-1772 (2016).

172. Simmons, L. A. et al. Comparison of responses to double-strand breaks between Escherichia coli and Bacillus subtilis reveals different requirements for SOS induction. $J$. Bacteriol. 191, 1152-61 (2009).

173. Elliott, S. J. et al. The Locus of Enterocyte Effacement (LEE)-Encoded Regulator Controls Expression of Both LEE-and Non-LEE-Encoded Virulence Factors in Enteropathogenic and Enterohemorrhagic Escherichia coli. INFECTION AND IMMUNITY 68, (2000).

174. Jiang, Y. et al. Multigene editing in the Escherichia coli genome via the CRISPR-Cas9 system. Appl. Environ. Microbiol. 81, 2506-14 (2015).

175. Zheng, L., Kelly, C. J. \& Colgan, S. P. Physiologic hypoxia and oxygen homeostasis in the healthy intestine. A Review in the Theme: Cellular Responses to Hypoxia. Am. J. Physiol. - Cell Physiol. 309, C350 (2015).

176. Ferraris, R. P., Yasharpour, S., Lloyd, K. C., Mirzayan, R. \& Diamond, J. M. Luminal glucose concentrations in the gut under normal conditions. Am. J. Physiol. 259, G822-37 (1990).

177. Riedel, T. E., Berelson, W. M., Nealson, K. H. \& Finkel, S. E. Oxygen consumption rates of bacteria under nutrient-limited conditions. Appl. Environ. Microbiol. 79, 4921-31 (2013).

178. Yang, B., Feng, L., Wang, F. \& Wang, L. Enterohemorrhagic Escherichia coli senses low biotin status in the large intestine for colonization and infection. Nat. Commun. 6, 6592 (2015).

179. Iyoda, S. et al. The GrlR-GrlA regulatory system coordinately controls the expression of flagellar and LEE-encoded type III protein secretion systems in enterohemorrhagic Escherichia coli. J. Bacteriol. 188, 5682-92 (2006).

180. McWilliams, B. D. \& Torres, A. G. EHEC Adhesins. Microbiol. Spectr. 2, EHEC00032013 (2014).

181. Neely, M. N. \& Friedman, D. I. Functional and genetic analysis of regulatory regions of coliphage H-19B: location of shiga-like toxin and lysis genes suggest a role for phage 
functions in toxin release. Mol. Microbiol. 28, 1255-1267 (1998).

182. O’Brien, A. D. \& Holmes, R. K. Shiga and Shiga-like toxins. Microbiol. Rev. 51, 206-20 (1987).

183. Hughes, D. T., Clarke, M. B., Yamamoto, K., Rasko, D. A. \& Sperandio, V. The QseC Adrenergic Signaling Cascade in Enterohemorrhagic E. coli (EHEC). PLoS Pathog. 5, e1000553 (2009).

184. Clarke, M. B., Hughes, D. T., Zhu, C., Boedeker, E. C. \& Sperandio, V. The QseC sensor kinase: a bacterial adrenergic receptor. Proc. Natl. Acad. Sci. U. S. A. 103, 10420-5 (2006).

185. Sperandio, V., Mellies, J. L., Nguyen, W., Shin, S. \& Kaper, J. B. Quorum sensing controls expression of the type III secretion gene transcription and protein secretion in enterohemorrhagic and enteropathogenic Escherichia coli. Proc. Natl. Acad. Sci. U. S. A. 96, 15196-201 (1999).

186. Carlson-Banning, K. M. \& Sperandio, V. Catabolite and Oxygen Regulation of Enterohemorrhagic Escherichia coli Virulence. MBio 7, e01852-16 (2016).

187. La Ragione, R. M. et al. Recombinant anti-EspA antibodies block Escherichia coli O157:H7-induced attaching and effacing lesions in vitro. Microbes Infect. 8, 426-433 (2006).

188. Kenny, B., Li, +, Lai, -Ching, Brett Finlay', B. \& Donnenberg, M. S. EspA, a protein secreted by enteropathogenic Escherichia coli, is required to induce signals in epithelial cells. Molecular Microbiology 20,

189. Weiner, N. \& Draskóczy, P. The Effects Of Organic Acids On The Oxidative Metabolism Of Intact And Disrupted E. coli. J. Pharmacol. Exp. Ther. 132, (1961).

190. Cherrington, C. A., Hinton, M., Mead, G. C. \& Chopra, I. Organic Acids: Chemistry, Antibacterial Activity and Practical Applications. Adv. Microb. Physiol. 32, 87-108 (1991).

191. Iyoda, S. \& Watanabe, H. Positive effects of multiple pch genes on expression of the locus of enterocyte effacement genes and adherence of enterohaemorrhagic Escherichia coli O157 : H7 to HEp-2 cells. Microbiology 150, 2357-2571 (2004).

192. Sharma, V. K., Kudva, I. T., Bearson, B. L. \& Stasko, J. A. Contributions of EspA Filaments and Curli Fimbriae in Cellular Adherence and Biofilm Formation of Enterohemorrhagic Escherichia coli O157:H7. PLoS One 11, e0149745 (2016).

193. Sperandio, V. et al. Activation of enteropathogenic Escherichia coli (EPEC) LEE2 and LEE3 operons by Ler. Mol. Microbiol. 38, 781-793 (2000). 
194. Haack, K. R., Robinson, C. L., Miller, K. J., Fowlkes, J. W. \& Mellies, J. L. Interaction of Ler at the LEE5 (tir) operon of enteropathogenic Escherichia coli. Infect. Immun. 71, 38492 (2003).

195. Barba, J. et al. A positive regulatory loop controls expression of the locus of enterocyte effacement-encoded regulators Ler and GrlA. J. Bacteriol. 187, 7918-30 (2005).

196. Bustamante, V. H., Santana, F. J., Calva, E. \& Puente, J. L. Transcriptional regulation of type III secretion genes in enteropathogenic Escherichia coli: Ler antagonizes H-NSdependent repression. Mol. Microbiol. 39, 664-678 (2001).

197. Rosenshine, I., Umanski, T. \& Friedberg, D. Thermoregulated expression of virulence genes in enteropathogenic Escherichia coli. Microbiology 148, 2735-2744 (2002).

198. Russell, R. M., Sharp, F. C., Rasko, D. A. \& Sperandio, V. QseA and GrlR/GrlA regulation of the locus of enterocyte effacement genes in enterohemorrhagic Escherichia coli. J. Bacteriol. 189, 5387-92 (2007).

199. Sharp, F. C. \& Sperandio, V. QseA directly activates transcription of LEE1 in enterohemorrhagic Escherichia coli. Infect. Immun. 75, 2432-40 (2007).

200. Sperandio, V., Li, C. C. \& Kaper, J. B. Quorum-sensing Escherichia coli regulator A: a regulator of the LysR family involved in the regulation of the locus of enterocyte effacement pathogenicity island in enterohemorrhagic E. coli. Infect. Immun. 70, 3085-93 (2002).

201. Islam, M. S., Shaw, R. K., Frankel, G., Pallen, M. J. \& Busby, S. J. W. Translation of a minigene in the 5' leader sequence of the enterohaemorrhagic Escherichia coli LEE1 transcription unit affects expression of the neighbouring downstream gene. Biochem. J. 441, 247-53 (2012).

202. Sánchez-SanMartín, C., Bustamante, V. H., Calva, E. \& Puente, J. L. Transcriptional regulation of the orf19 gene and the tir-cesT-eae operon of enteropathogenic Escherichia coli. J. Bacteriol. 183, 2823-33 (2001).

203. Zhu, C., Feng, S., Thate, T. E., Kaper, J. B. \& Boedeker, E. C. Towards a vaccine for attaching/effacing Escherichia coli: a LEE encoded regulator (ler) mutant of rabbit enteropathogenic Escherichia coli is attenuated, immunogenic, and protects rabbits from lethal challenge with the wild-type virulent strain. Vaccine 24, 3845-3855 (2006).

204. Yerushalmi, G., Nadler, C., Berdichevski, T. \& Rosenshine, I. Mutational Analysis of the Locus of Enterocyte Effacement-Encoded Regulator (Ler) of Enteropathogenic Escherichia coli. J. Bacteriol. 190, 7808-7818 (2008).

205. Berdichevsky, T. et al. Ler Is a Negative Autoregulator of the LEE1 Operon in 
Enteropathogenic Escherichia coli. J. Bacteriol. 187, 349-357 (2005).

206. Flockhart, A. F. et al. Identification of a novel prophage regulator in Escherichia coli controlling the expression of type III secretion. Mol. Microbiol. 83, 208-23 (2012).

207. Turner, N. C. A., Connolly, J. P. R. \& Roe, A. J. Control freaks-signals and cues governing the regulation of virulence in attaching and effacing pathogens. Biochem. Soc. Trans. 47, 229-238 (2018).

208. Ramos, A. et al. Altered morphology produced by ftsZ expression in Corynebacterium glutamicum ATCC 13869. Microbiology 151, 2563-2572 (2005).

209. Johnson, M. Antibody Shelf Life/How to Store Antibodies. Mater. Methods 2, (2012).

210. Schüller, S. \& Phillips, A. D. Microaerobic conditions enhance type III secretion and adherence of enterohaemorrhagic Escherichia coli to polarized human intestinal epithelial cells. Environ. Microbiol. 12, 2426-35 (2010).

211. Macfarlane, G. T., Gibson, G. R. \& Cummings, J. H. Comparison of fermentation reactions in different regions of the human colon. J. Appl. Bacteriol. 72, 57-64 (1992).

212. Schuchardt, J. P. \& Hahn, A. Intestinal Absorption and Factors Influencing Bioavailability of Magnesium-An Update. Curr. Nutr. Food Sci. 13, 260-278 (2017).

213. Rude, R. K. Magnesium Deficiency: A Cause of Heterogenous Disease in Humans. $J$. Bone Miner. Res. 13, 749-758 (1998).

214. Lv, Y. et al. Systematic mutation analysis of two-component signal transduction systems reveals EsrA-EsrB and PhoP-PhoQ as the major virulence regulators in Edwardsiella tarda. Vet. Microbiol. 157, 190-199 (2012).

215. Chakraborty, S. et al. Temperature and Mg2+ sensing by a novel PhoP-PhoQ twocomponent system for regulation of virulence in Edwardsiella tarda. J. Biol. Chem. 285, 38876-88 (2010).

216. Tao, H., Bausch, C., Richmond, C., Blattner, F. R. \& Conway, T. Functional genomics: expression analysis of Escherichia coli growing on minimal and rich media. J. Bacteriol. 181, 6425-40 (1999).

217. Albenberg, L. et al. Correlation Between Intraluminal Oxygen Gradient and Radial Partitioning of Intestinal Microbiota. Gastroenterology 147, 1055-1063.e8 (2014).

218. Dharmarha, V. et al. Effect of post-harvest interventions on surficial carrot bacterial community dynamics, pathogen survival, and antibiotic resistance. Int. J. Food Microbiol. 291, 25-34 (2019). 
